\title{
E. UTEUBAYEVA
}

\section{CONCEPTUAL \\ FRAMEWORK FOR \\ TEACHING PROFESSIONAL \\ FOREIGN LANGUAGE (CASE OF LSP FOR LAW):}

for universities studying English

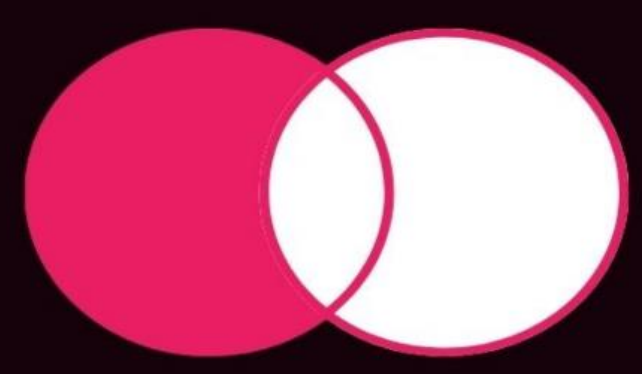

VELIKO TARNOVO

2021 


\section{ELMIRA UTEUBAYEVA}

\section{CONCEPTUAL FRAMEWORK FOR TEACHING PROFESSIONAL FOREIGN LANGUAGE}

(CASE OF LSP FOR LAW)

/for universities studying English/

MONOGRAPH

VELIKO TARNOVO, 2021 
(C) Elmira UTEUBAYEVA, author, 2021

\section{REVIEWERS:}

Gulnara SARSENBAYEVA, PhD (Ed), University of Languages and Professional Career, Almaty city, Republic of Kazakhstan

Anarbek KOZYBAY, Doctor of Pedagogical Sciences, Professor, Abai Kazakh National Pedagogical University, Almaty city, Republic of Kazakhstan

Antonina KIZDARBEKOVA, Candidate of Legal Sciences, Associate Professor, Academician E.A.Buketov Karaganda University, Karaganda city, Republic of Kazakhstan

\section{EDITOR:}

Dzintra ILIŠKO, Professor, Dr

Daugavpils University, Latvia

Institute of Humanities and Societal Sciences, Centre of Sustainable Education

ISBN 978-619-91511-3-6

(C) Publisher: ACCESS Press Publishing house, 2021. 180 p.

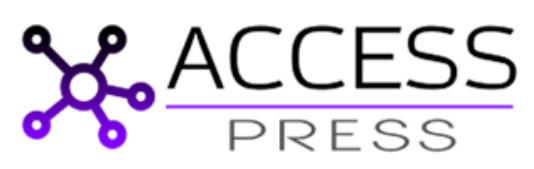

Published under the terms of the Creative Commons

CC BY-NC 4.0 License 
The monograph is intended for researchers and students of English for special purposes. The main goal of the monograph is to impart the theory of foreign language proficiency and the formation of language competence. The monograph provides a conceptual framework consisting of theoretical and methodological approaches aimed at the formation of the language and requirements for the methodology of teaching English for special purposes using the example of English for lawyers. The monograph consists of two parts, the first part critically examines the theory of learning, the second part analyzes the postgraduate study program and concludes with guidelines for teaching English for special purposes.

Uteubayeva, E. (2021) CONCEPTUAL FRAMEWORK FOR TEACHING PROFESSIONAL FOREIGN LANGUAGE (CASE OF LSP FOR LAW): for universities studying English. / Ed. Iliško, Dz., ACCESS Press Publishing house, Veliko Tarnovo, Bulgaria, 2021. 180 p.

\section{ISBN 978-619-91511-3-6}

https://doi.org/10.46656/book.2021.Uteubayeva

(C) ACCESS Press Publishing house, 2021

5000 Veliko Tarnovo, Bulgaria

E-mail:office@access-bg.org

http://www.access-bg.org 
CHAPTER 1. THEORY OF LANGUAGE LEARNING

1.1 RESEARCH ANALYSIS ON LANGUAGE COMPETENCE 7 DEVELOPMENT PROBLEMS

1.2 METHODOLOGICAL FRAMEWORK IN FOREIGN 35 LANGUAGE PROFICIENCY

CHAPTER 2. EMPIRICAL RESEARCH ON METHODS OF FOREIGN LANGUAGE TEACHING

2.1 ANALYSIS OF METHODS OF TEACHING FOREIGN LANGUAGES FOR SPECIAL PURPOSES IN POSTGRADUATE EDUCATION PROGRAM

2.2 METHODS OF TEACHING ESP: RECOMMENDATIONS AND DISCUSSIONS 


\section{INTRODUCTION}

Kazakhstan's entry into the world community, establishment of democratic values and development of a market economy pose a difficult task for the education system to train competitive specialists who can creatively comprehend the processes taking place in the republic and professional sphere, navigate the rapidly expanding flow of scientific information. This fully applies to the problems of modern legal theory and practice, development of which is due to the orientation of the legislative and regulatory framework of the Republic of Kazakhstan towards integration with the world community, functioning of international information databases on jurisprudence.

In accordance with the State program for the development of education and science of the Republic of Kazakhstan for 2020 - 2025, which presupposes that the modernization of higher and postgraduate education will be carried out in the context of global and interdisciplinary competences of the 21 st century. The determination of strategic priorities in the development of a national model of multilevel continuing education, the content of learning at each level of education is based on different goals and expected results, not united by a common methodological framework. There is no single concept of lifelong learning, which defines the model (image) of a citizen of Kazakhstan with the necessary set of knowledge, skills and competencies. A modern Kazakhstani professional in Law should receive not only legal knowledge, but also opportunities for the development of tactical, communicative and psychological skills in the context of expanding international cooperation and cultural globalization. New educational programs will focus on the formation of flexible and professional skills (soft skills, hard skills) required in the rapidly changing world of VUCA (Volatility, uncertainty, complexity, and ambiguity), involving lifelong learning.

It was these processes that dictated the need to develop the concept of lifelong education, which implies regular updating and expansion of the knowledge base throughout life. This concept was adopted by the Council of European Commission on Higher Education and Research in the framework of the program "University of Tomorrow: Politics of Higher Education in Europe". It is connected with the constant staff development and improvement of the qualifications of specialists.

The analysis of a number of Council of Europe documents on education and vocational training of specialists indicates that a purposeful policy is being pursued in the Pan-European Community, focused on the formation and development of specialists' abilities to communicate freely in a foreign language in the field of professional activity. This situation is projected onto the problems of 
communication in the work of a lawyer, which plays a primary role in his professional activity, since it inevitably involves interaction with other people. Only in the process of interpersonal communication are negotiations between a lawyer and interested parties carried out, court cases are considered, opinions and decisions of the competent authorities and officials are communicated to the addressees. Therefore, the training of a lawyer must necessarily be guided by the development of their communicative competence, based on the formation of skills of professionally oriented communication in their native and foreign languages. The formation of the types of speech activity determined by the subject area of lawmaking and law enforcement requires a transition from informative and reproductive methods of teaching foreign languages to problem-developing, creative-search, pragma-professional technologies of foreign language teaching. The most important condition for the successful formation of a foreign language communicative competence of a lawyer in the system of postgraduate education is taking into account their scientific interests and motives of education.

Purposeful acquaintance with the experience of law universities, study of the initial state of training of lawyers at the stage of postgraduate education in educational institutions of the National Security Committee and the Ministry of Internal Affairs of the Republic of Kazakhstan allow us to state that the methodology of teaching professionally oriented foreign language communication needs to be updated and improved, since there is an extreme lack of modern alternative textbooks, teaching aids and methodological developments, reflecting not only the specifics of professionally oriented communication in a foreign language of legal professionals, but aimed at forming the linguistic personality of a specialist ready for intercultural communication taking into account the latest theoretical achievements of the methodology and related sciences. This is due to the inertial lack of demand for a foreign language in the professional activities of lawyers, insufficient development of methods of level teaching of a foreign language, taking into account the specifics of foreign language training of lawyers in the system of postgraduate education.

In general, the study of problems of foreign language communicative competence development has a certain tradition. At the same time, this problem in the context of professional training of lawyers in the system of postgraduate education needs searching optimal, effective, scientifically based solutions in the development of appropriate didactic support. 


\section{CHAPTER 1. \\ THEORY OF LANGUAGE LEARNING}

\subsection{RESEARCH ANALYSIS ON LANGUAGE COMPETENCE DEVELOPMENT PROBLEMS}

In the mid-80s of the last century the Russian philosopher M. Mamardashvili (Mamardashvili, 2019) analyzed the relationship between national and universal human components in the culture of mankind and discovered that knowledge of any language other than national language is necessary for the individual to the extent that this foreign language opens the way for a person to world culture, creativity and freedom. We have to admit that under the new conditions of independence the society has gained more freedom and new human relationships have been established.

To achieve the goal of the creation of a welfare society based on a strong state, a developed economy and the possibilities of universal labor, Kazakhstan's entry into the thirty most developed countries in the world, "Strategy Kazakhstan2050" foresees the implementation of one of the long-term priority, that the knowledge and professional skills are the key guidelines of the modern education system, training and retraining of personnel (Nazarbayev, 2012).

In the course of the processes of globalization, cultural, socio-economic, legal, scientific and educational contacts with representatives of other linguistic societies are being evaluated in a new way. These contacts, exchange of experience are impossible without the mediation of a foreign language as a means of carrying out intercultural communication, not only of a private but also of a professional nature. The need for specialists in a particular field of human activity is increasing every year. Therefore, research in the field of a professional foreign language is necessary to solve potential problems arising in the process of communication between specialists of different linguistic cultures in a particular professional field.

In this regard, the question of proficiency of a foreign language for professional purposes is highly relevant in the post-industrial world. The increasing trends of globalization promote cross-cultural exchange between people, and, first, these exchanges are of an interprofessional nature, including the field of education. The process of increasing integration of Kazakhstani universities into the world education system is being observed. More and more postgraduates, doctoral students, teachers go abroad for internships and the means of communication in this case is the language of the host state.

The result of the integration process is, firstly, an increase in the motivation for learning a foreign language in one's own university, secondly, an update of 
approaches to teaching and learning a foreign language, and thirdly, a personal growth of subjects of study.

The great attention is paid to the development of the intellectual potential of science in the State program for the development of education and science of the Republic of Kazakhstan for 2020 - 2025. As it was noted, that there is a problem of attracting and retaining talented youth and highly qualified specialists in science. Available scientific personnel have insufficient research skills, low proficiency in English and weak skills in technological development and innovation (Government program of development of education and science of the Republic of Kazakhstan for 2020 - 2025, 2019).

Thus, the structure and content of the education system as a whole, both general, special and postgraduate, is certainly determined by the socio-political aspirations of society and its value orientations. The formation of civil society and the building of the rule of law require a radical change in the content of education, further optimization of theory, forms and methods of teaching, in particular foreign languages at the postgraduate stage. According to M. Halbwax (Krubelye, 1989) education is considered as the most effective means by which the state forms people in its likeness.

Kazakhstani society, seriously and irrevocably standing on the path of global transformations is currently forming a new social order for the entire system of domestic education, to raise a person who is free, widely and independently thinking, and capable of intellectual search and creative solutions. This is not about the formation of intellectual elite, a limited "caste" of geniuses, carefully stamped by the state in the name of solving the acute problems facing our country: this is theoretically unlikely, but almost ineffective. So, the greatest French materialist philosopher D. Diderot in the XVII century drew a pragmatic line under similar eugenic tasks noting that geniuses forced to feel and decide only to their liking, in their disgust, very guessed, little foreseeable, seems to be more suitable for tipping the old, states than to maintain them; more suitable for establishing order than for following it (Efroimson, 2009). The most adequate to the modern state of Kazakh society is the understanding of the goals of education, formulated by B. Rassell (2014) who believed that education should have two goals. The first is to give certain knowledge and the second is to create those mental habits that will allow people to acquire knowledge and make their own opinions. Education gives an understanding of the value of things that are not related to power, helps to educate wise citizens of a free society and by combining citizenship with freedom in individual creativity, gives people the opportunity to introduce into human life the greatness that few have managed to achieve (Ilyichev, Fedoseev \& Kovalev, 1983). 
Globalization and modernization make the world more and more diverse and saturated with information networks and technologies. In order to understand this world and navigate well in it, people must understand technological changes, large amounts of information and use them wisely. The competencies that we need today to achieve our goals must be complex, i.e., possession of several separate abilities and performing skills is insufficient. Continuous development and social ties clearly depend on the competences of the entire population, where the concept of competence means "knowledge, skills, life affirmations and values" (OECD, 2005a).

The freedom of the individual is seen as the ability of a person to act in accordance with his interests and goals, based on the knowledge of objective necessity (Ilyichev, Fedoseev \& Kovalev, 1983). Professionalism is what we understand as the ability of a person based on special knowledge and skills to effectively solve a range of certain tasks and realize professional goals. Thus, individual freedom and professionalism are mutually reinforcing, that is, ambivalent in a certain sense. They practically differ from each other only in the degree of completeness of the reflection of reality (freedom is correlated in general with the surrounding world - professionalism is essentially freedom of choice in a limited segment of the latter) and the absence (presence) of a choice of qualitative certainty, freedom is the ability of a person to act - professionalism appears to be the ability of a specialist to act optimally). The obvious evidence of the understanding of the essential and functional relationship of the concepts under consideration is that one of the most significant state policy priorities is the creation of a compact professional government, the attributes of which will be the ability to strategic planning, financial programming and project management. In addition, the head of our state sees the most important condition for solving this problem in the creation of a powerful and effective system for training managerial personnel.

An inevitable by-product of the massification of higher education is that a significant number of graduates are not finding the jobs they expected. There are also graduates who are unable to perform at the level of work expected of them. This is not just a matter of employment, but also a matter of motivation and expectations. Many employees are required to communicate in English. Others must be able to work in different cultural contexts. They need the communication and speech skills that are increasingly expected of graduates, regardless of their subject area. A large number of managers in different sectors were asked the question: is possession of a degree a necessary or necessary prerequisite for hiring; many have moved or are moving from qualification requirements to competency requirements, including general skills (such as communication and teamwork skills) and personal qualities (such as resilience and commitment). Accordingly, future law enforcement 
officers should have a wide range of skills and specific knowledge, namely analytical skills, social skills, management, communication and networking skills, learning ability, knowledge of foreign languages (mainly English), presentation skills, lifelong learning strategies, intercultural communication skills.

In details, from the sources of the accreditation agency (NVAO, where seven areas of competence have been identified that characterize the university postgraduate (TU Delft et al 2005). In short, the graduate is:

1) competent in one or more scientific disciplines (and is able to develop this competence through studies);

2) competent in conducting scientific research (and has the competence to acquire new scientific knowledge through conducting research).

3)competent in planning;

4) knows how to use a scientific approach (critical attitude, understanding of the nature of science and technology);

5) possesses basic intellectual skills (argumentation, reflection, formation of judgments);

6) competent in cooperation and communication (such competencies as the ability to work with others and for others, a sense of responsibility, the ability to be a leader, to communicate with colleagues and non-colleagues).

7) takes into account temporal and social contexts (awareness of social consequences and the ability to take these ideas into account in scientific work) (Arthur, Brennan \& Weert, 2007).

It should be noted that discussions on ways to increase the level of professional competence of a specialist from the traditional field - pedagogy (Kuzmina, 1990; Molchanovskii, 2002) are steadily moving to new professional areas, until recently relatively "indifferent" to the analyzed issues. For example, the study of the nature of the auditor's professional competence, the process of becoming a professional and education in this field is devoted to the study of American scientists T. Hassal and A. Dunlop (1996). Issues of development of professional competence of managerial personnel are considered in works of the vice-rector of the Russian Academy of Management and Market B.T. Ponomarenko.

Most of the articles of the scientific collection "Questions of Psychology" (1990), etc. are devoted to determining the components of a professional competence model of a branch psychologist and ways to optimize it. Moreover, despite the obvious difference between these areas of human activity (audit, pedagogy, psychology, management theory, etc.), all authors agree in one significant position that the constitutional component of the professional 
competence of a specialist, according to researchers, is communicative, in particular foreign-language competence.

In pedagogy the concept of competence as in other humanities, came from business, where a competent approach is associated with the name of David Mack Clelland. This concept is very widespread in jurisprudence, more precisely in the field of legal relations. In modern legal dictionaries, it is interpreted as a set of rights and obligations established by normative acts of organizations, bodies, officials, as well as persons performing managerial functions in commercial organizations (Encyclopedia of Private International Law, 2017). In nature science, "competence" has a wider conceptual field and means the ability of a living organism, joint activity, external irritation, reaction - reflection, as well as the result of reflection (Saharova, 1999).

The general context of the definition of the concept under consideration correlates with the professional activity of a person. Pedagogical science and modern practice of education in this sense are not an exception. Here it gave rise to a completely methodologically significant concept of a competent approach.

In the last 20-25 years, many educational systems have been embraced by the movement of the competence-based approach, from school to university. This approach is based on a deviation in understanding the ultimate goals of education. The competence-based approach tried to redirect these goals towards the mobilization of knowledge, because the development of "dead knowledge" was observed, i.e. knowledge that the postgraduate was able to understand and perceive, remaining, however, unable to activate it in situations where the application of this knowledge was appropriate (inability to mobilize what was learned in the real world). With a competency-based approach, we try to convey to postgraduate ways to think and act in the world around them.

The university's transition to a competency-based approach generates three breakthroughs in the way we understand education:

- $\quad$ Teachers are required to define their goals.

- $\quad$ These goals should henceforth be formulated in terms of the knowledge that the student must acquire, while the university learning paths are generally conceived according to the internal logic of the discipline content.

These goals should be expressed in terms of competence acquisition, i.e. based on the means / ways for acting and thinking in the world that the postgraduate is supposed to manage upon leaving the education system.

This is even more new because traditionally, university education was theoretical, and the application and mobilization of knowledge in reality was seen as an addition (Romenville, 2004). 
The study of this question's background allowing us a better understanding of its pedagogical essence encompasses the transformation of knowledge, skills and abilities into professional competencies, first implying high quality professional skills. (Goncharova, 2007).

Directly in the professional education of teachers, this approach began to be applied first in the USA (Houston.\& Howsam, 1972), and then in Soviet pedagogy (Vvedenskii, 2003), which, with its inherent gravitation towards fundamentality, gave this concept a wide content.

Therefore, based on N.V. Kuzmina's classification in the content of professional competence of the teacher we differentiate between the following criteria such as: professional, methodological, socio-psychological, differential and psychological and auto psychological competencies (Kuzmina, 1982).

Because of the individual's self-development, personal growth and enrichment of personal experience as a way to knowledge and skills existence contributing to personal self-realization professional competence is considered as the most effective one (Bolotov \& Serikov, 2003).

In addition, the essence of professional competence as a combination of abilities, qualities and personality properties necessary for successful professional activity is interpreted by another research (Klimenko, 2003).

In a similar way, the meaning of this term is explained that the competence of a specialist is a set of integrated fundamental knowledge, generalized skills, and abilities of a person, his professionally significant and personal qualities, and a high level of technological effectiveness, culture and skill, a creative approach to organizing activities, readiness for constant self-development (Kenzhebekov, 2002).

The professional competence of future teachers is explained as the qualitative characteristics of the personality of the future teacher, as a subject of professional activity, manifested in the gnostic, procedural, communicative, personal, creative, and reflective components, integrated into the experience of practical activity, the basis of which is the orientation of the personality (Semenova, 2005).

Based on the activity and sociocultural approaches, the essence of the teacher's professional competence is determined by Markova A.K., since in her understanding of this category, the classification of pedagogical skills is the basis. In accordance with this, she distinguishes special (mainly professional), social, personal and individual competence, from which it follows that she considers professional competence as part of socio-cultural competence (Markova, 1996).

Competence is also considered as a complex of personal professionally significant qualities, theoretical knowledge and practical skills, confirmed by the level of initial education (Taubayeva, 2005). 
Professional competence comprehends as the readiness and ability of a person to make effective decisions in the implementation of professional activities, to design and carry out these activities in interaction with the outside world. The formation of this readiness can be facilitated by a set of integrated knowledge, skills and experience, as well as personal qualities. Determining the signs of professional activity, characteristics of the current interest and potential activities of a specialist is grouped (Shaposhnikov, 2006).

The definitions of professional competence is identical to the possession of professional knowledge and skills. (Karapetova, 2000; Bezrukova, 1996).

Noting the same components (professional knowledge, abilities, skills), it is defined that they are formed by means of the content of education (Hutorskoi, 1998).

While discussing the content of a professional competence the following structural components are offered such as: 1) objectively necessary knowledge, abilities, skills; 2) professional positions, largely due to the orientation of the individual, which is a whole system of needs, values, aspirations, meaning-forming motives; 3) individual psychological characteristics of a person, on which the style of activity and professional behavior depend (Abulhanova - Slavskaya, 1980).

Pedagogical skills are defined of particular importance in the composition of professional competence. For example, to "translate" the content of the objective process of education into specific pedagogical tasks, build and put into action a logically complete pedagogical system, highlight and establish relationships between the components and factors of education, accounting, and evaluation of learning outcomes pedagogical activity (Slastenin, 1996).

It follows from this that the preference is given mainly to organizational (mobilization, information, developmental, orientation skills) and communicative (perceptual skills, pedagogical communication skills, and pedagogical technique) skills of the teacher. In general, pedagogical skills are considered as sequential actions aimed at solving pedagogical problems (Slastenin, 1996).

Also, in the scientific literature, there are concepts that are associated with the allocation of universal components of professional competence, which can be fully correlated with the professional activities of teachers. As such, there are axiological (1), cultural (2), life-creating (3), moral and aesthetic (4), civil (5).

Their signs are defined like this:

1) The first component is characterized by a spiritual principle; it is represented by universal values;

2) The second component covers the values and traditions of the national culture, as well as the general cultural abilities of a person, which are necessary for him in professional activity; 
3) The third component includes the ability and readiness to transform the micro society;

4) The fourth component contains the psychological experience of the individual (experiences and life in emotionally saturated situations; humane behavior; tolerance towards other people; adequate self-esteem);

5) The fifth component reflects the experience of human civic behavior (Semenova, 2007).

The most fully studied concept is revealed in various classifications of types of competencies.

Thus, in the literature meets the classification, according to which should be allocated to ordinary (basic) and key competences. The first of which is formed on the basis of knowledge, skills, abilities, easily fixed, manifested in certain activities, the second - a very difficult to account for and measure, are manifested in all types of activity, in all respects of the personality, reflect its spiritual world and the meanings of its activities.

Another classification is directly related to pedagogical activity, as it highlights such competencies as:

- $\quad$ business (making pedagogical decisions)

- $\quad$ special (knowledge of the taught school discipline);

- $\quad$ social (ability and readiness for pedagogical interaction);

- $\quad$ methodological (the ability to develop curricula and projects);

- personal.

Scientists at Frey, Balzer \& Renold mean by professional competence physical and spiritual reserves, i.e. the potential that a person needs as a prerequisite for a responsible and purposeful solution of emerging tasks or problems, for assessing the solutions found and further developing their own approach to typical actions.

This requires a range of professional, psychological, and personal dimensions from the individual (Frey, Balzer \& Renold, 2002).

In our understanding, this is represented as follows in Figures 1, 2, 3, 4: 


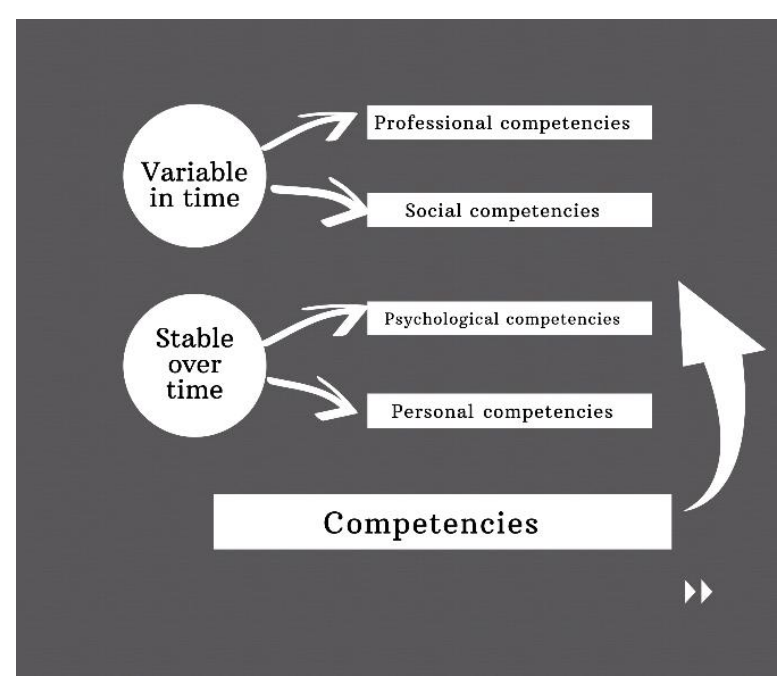

Fig.1 Classification of competencies

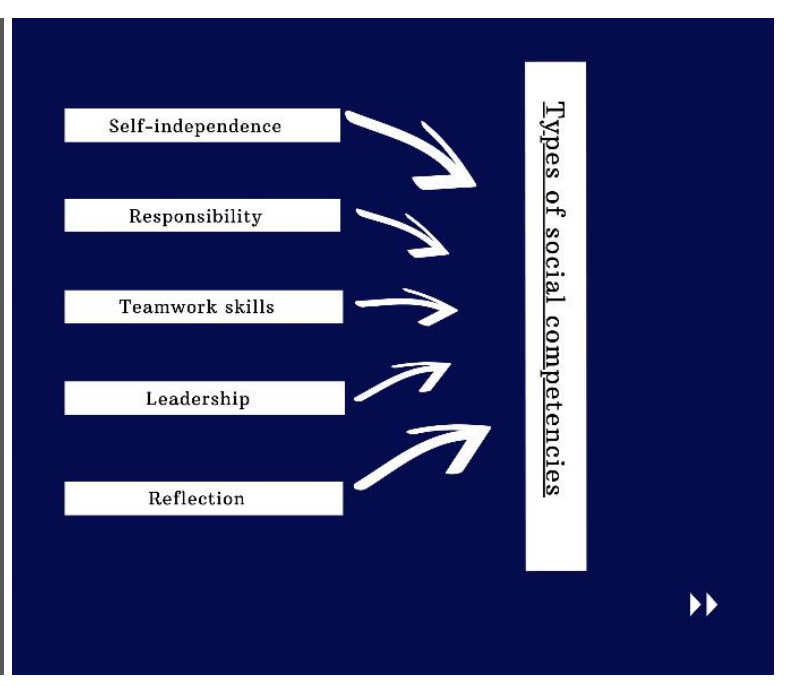

Fig.2 Types of social competencies

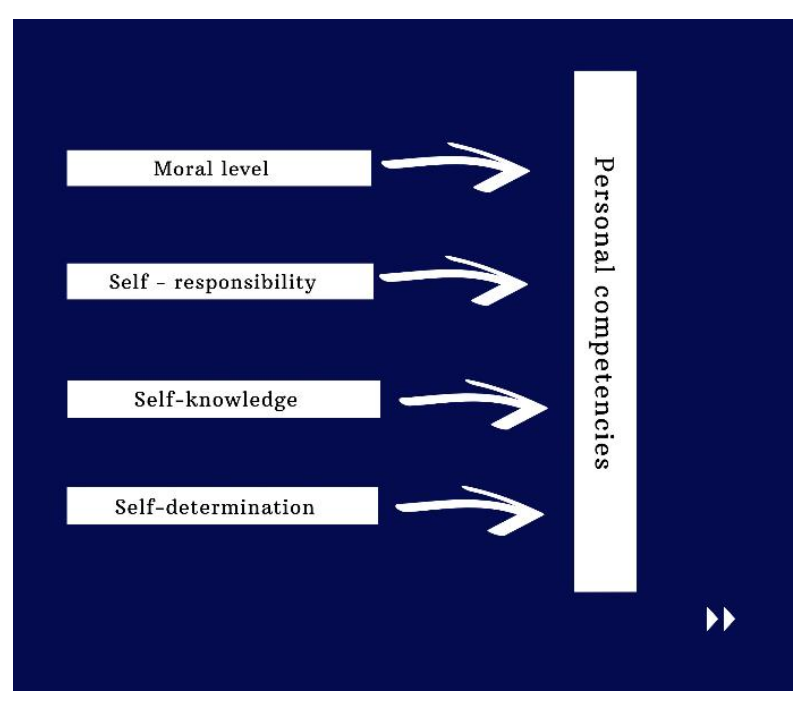

Fig.3. Types of personal competencies

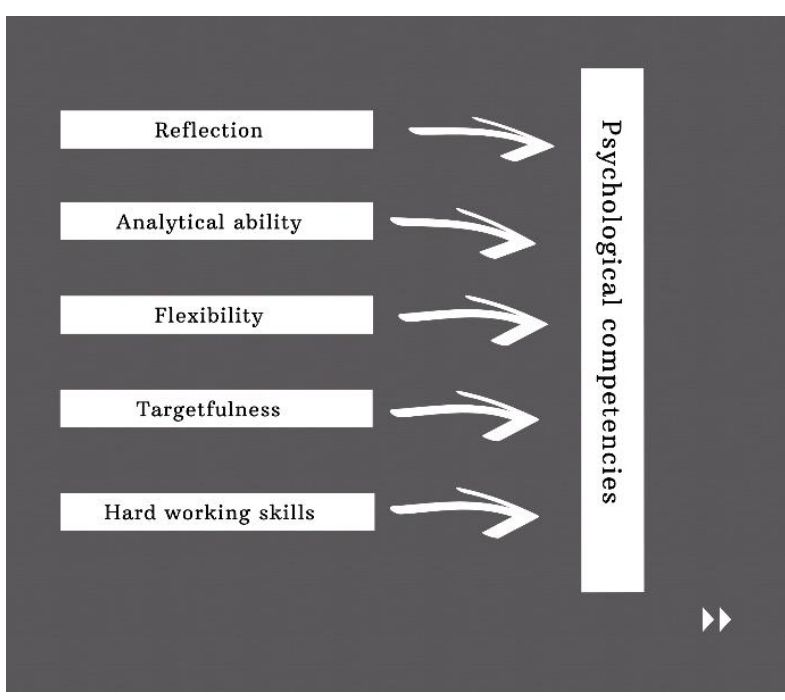

Fig.4. Types of psychological competencies

According to the following classification, they differ: standard (without them, normal functioning of an individual or organization is impossible), key (they ensure a person's competitiveness in the socio-economic market), leading (they are manifested in innovativeness, creativity, dynamism and dialogue) (Bazarov, n.d).

The classification of competencies is also interesting, which draws attention to the following competencies as adaptive and civilizational, social, professional, social and organizational, communicative and value-semantic (Isaeva, n.d.]. This classification belongs to T.E. Isaeva, who later developed a number of professional and pedagogical competences according to the "to know, to do, to be" scheme (Isaeva, 2003].

Since the 1970s, the purpose of second and foreign language teaching has aimed at developing communicative competence. The communicative competence 
concept was born in opposition to the previous approaches being used that merely focused on grammar and the correctness of the language.

Hymes (as cited in Brown, 2000) defined communicative competence as the aspect of the competence that enables us to convey and interpret messages and to negotiate meanings interpersonally within specific contexts (Brown, 2000). From this point of view, the communication process was not an individual process anymore; it turned into a process where all speakers, listeners, writers and readers played an active role. They all worked cooperatively to understand and be understood.

Based on Hymes' concept of communicative competence, Canale and Swain (as cited in Brown, 2000) proposed four different categories: grammatical, discursive, sociolinguistic and strategic competences (Brown, 2000).

Brown (2001) presented an updated version of communicative competence which summarizes the concepts presented by Canale and Swain and other linguists.

In its skeletal form (Zheng, Perez, Williamson \& Flygare, 2008), CC [communicative competence] consists of some combination of the following components:

- $\quad$ Organizational competence (grammatical and discourse)

- $\quad$ Pragmatic competence (functional and sociolinguistic)

- $\quad$ Strategic competence

- $\quad$ Psychomotor skills (pronunciation).

In general, competence in the scientific literature is considered as the result of professional training. We, fully accepting this position, draw attention to the fact that communicative competence is a constituent component of a specialist's professional competence. In the context of our research, this is projected onto foreign language communicative competence.

Competence is interpreted more broadly than human knowledge and cognitive abilities. We are talking about a person's ability to possess complex abilities and use them in a specific context, including psychosocial resources (for example, thinking abilities, attitudes and ways of behavior). For example, communicative ability is a personality's competence based on linguistic knowledge and skills, practical knowledge of information technology and interpersonal relationships in the process of communication (OECD Project, 2005).

Evidence of the importance of highly qualified law enforcement officers' training was mentioned by the former Minister of Internal Affairs of the Republic of Kazakhstan Z.K. Turisbekov: "A young man who decides to devote his life to a difficult service in law enforcement agencies must not only acquire the necessary legal knowledge, but also be smart in all respects." Education should be focused on practice, the development of tactical, communication and psychological skills 
(Turisbekov, 2004). A significant step towards optimizing higher departmental education was the adoption of the Concept for the development of departmental education of the Ministry of Internal Affairs of the Republic of Kazakhstan. To prioritize at the head of the learning process "the use of problem-based learning as an effective form of organizing and conducting the educational process, which allows each student to be given, along with high theoretical training and practical skills necessary in order to be able to solve problems within the sphere of professional activity (On the Concept for the development of departmental education of the Ministry of Internal Affairs of the Republic of Kazakhstan, 2004).

In projection to the investigated problem, this task, among the designated skills, actualizes the problem of the formation of a foreign language communicative competence of lawyers, therefore, the problem of a close connection between jurisprudence and language. Understanding by the domestic legislator of this connection follows from the rule of paragraph 1 of Art. 6 of the Civil Code of the Republic of Kazakhstan ("Interpretation of the norms of civil legislation"): "The norms of civil legislation should be interpreted in accordance with the literal meaning of their verbal expression (Civil Code of the Republic of Kazakhstan dated December 27, 1994). Those who study the problems of law enforcement interpretation in Kazakhstani legislation, the principle of the completeness of the use of methods and methods of interpretation means the implementation of interpretation with the help of the entire system of technical means of understanding the meaning of the rule of law, regardless of the level of legislative technique and the quality of the text of the normative act (Orumbayev, 2004). "Simple" and "clear" law requires, specifically grammatical (linguistic) interpretation, which presupposes a high level of broadly communicative competence of the law enforcement officer.

The analysis of the structure of professional activity of a lawyer made it possible to single out it as a substructure of the communicative function. One of the most important qualities of this activity is the ability to establish interpersonal contacts with various participants in communication, which greatly affect the efficiency of a lawyer's work.

Therefore, in addition to knowledge of laws, a lawyer also requires certain communication skills: the ability to establish relationships with people, persuade, taking into account individual psychological characteristics, to awaken interest in communication in connection with certain circumstances that have legal significance.

The professional training of lawyers presupposes the formation of the communicative competencies necessary for their practical activity, since the profession of a lawyer, according to a number of researchers, is one of the professions with "increased speech responsibility". 
Awareness of the inextricable link between jurisprudence and language, lawmaking and law enforcement, on the one hand, and the level of the communicative competence of the legislator and law enforcement officer, on the other hand, contributed to the fact that at present in the legal science of the CIS countries were developed new ones, "borderline" industries (on intersection of linguistics and jurisprudence): lingua jurisprudence and legal linguistics (Golev, 1999). These branches of science consider the language, native speakers, first, thereafter, as a subject and object of law; secondly, as a means, on the one hand, of the creation and understanding of the law (legislative and interpretive functions of natural language in the legal sphere) and, on the other hand, of the application of the law, where language is the subject (or means) of examination (linguistic expert function of practical knowledge language and theoretical knowledge of the language, requiring recourse to special linguistic competence).

Moreover, the consolidation in paragraph 2 of Art. 14 of the Constitution of the Republic of Kazakhstan, the principle of the categorical prohibition of "any discrimination based on origin, social, official and property status, gender, race, nationality, language (italics ours - E.U.), attitude to religion, beliefs, place of residence or any other circumstances" (Constitution of the Republic of Kazakhstan dated August 30, 1995) and in Art. 4-7 of the Law of the Republic of Kazakhstan "On Languages in the Republic of Kazakhstan" - functional bilingualism (polylingualism) (Law of the Republic of Kazakhstan "On languages in the Republic of Kazakhstan, 1997) increase the level of requirements for the communicative competence of a law enforcement officer in general and foreign language communicative competence in particular.

It should be noted that the tendency to increase the requirements for the level of communicative, primarily foreign language, competence of a police officer of the Republic of Kazakhstan is, in our opinion, a component of the common Eurasian processes of cultural integration. UNESCO had declared the 2001-st year as the European Year of Languages in Vienna (Austria). The keynote of International conferences "The Price of Monolingualism" and "The Future of a Multilingual Europe" was the statement of the fact that multilingualism is an integral part of the present and future Europe and that proficiency in several foreign languages is currently determined not only by economic, but to a greater extent by general educational moments, as a factor in the general political and cultural development of the individual. Therefore, at the present stage of the development of society, the culture-forming function of education is increasing, which, from a method of enlightenment, should turn into a mechanism for the development of culture, the formation of the world and the person in it. 
The interrelationship between language and culture are so close that are being identified as synonyms (Scarcella,1992). On the one hand, language is used to express people's cultural thoughts, beliefs and to communicate; on the other hand, culture is embedded in the language. The interwoven relationship between language and culture can be summarized by Brown (Brown, 2000) that a language is a part of a culture and a culture is a part of a language; the two are intricately interwoven so that one cannot separate the two without losing the significance of either language or culture.

Both of language and culture have a function of communication because they both carry meanings. On the one hand, language carries syntactic, semantic and pragmatic meanings for language users to communicate (Brooks,1997). On the other hand, culture carries meanings and cultural meanings are expressed through patterns of behavior, e.g., language. In order to communicate successfully across languages and cultures, one must understand culturally different norms of interaction and people's values and thought (Saville - Troike,2003). Sometimes linguistic correct sentences could cause misunderstanding or confusion when they are in a different cultural context (Schultz, 2007).

Thus, education in modern Europe is understood as the self-awareness of civilization that is, mastering the foundations of philosophy, science and the most important works of art and literature. Thus, the main task of education is to form and develop a personality, but not through the accumulation of certain facts, but through the assimilation of a certain content, primarily through reading (reading in the language of the original source - E.U.). The most important thing in the concept of education in Europe is that an educated person is a product of his culture, which allows him to be tolerant. Tolerance of an educated person is understood not only as forced tolerance, but also as recognition of another worldview as equal, awareness of one's own boundaries, recognition of another personality and culture as a necessary condition for enriching one's own existence in the modern world, in which the gradual rapprochement of different peoples is taking place" (Berdichevsky, 2002). Thereby, modern Western pedagogy postulates as one of the main means of harmonizing interpersonal and interethnic relations mass polylingualism, a significant increase in the foreign language communicative competence of a modern European.

To solve this problem, it is necessary to reform the traditional system of teaching a foreign language, the main directions of which are the following: early learning of a foreign language, taking into account the neighboring languages and related languages;

use of a foreign language as a means of teaching (bilingual, immersion training, in which various subjects are taught both in the native language and in a 
foreign language) with a simultaneous reduction in the time spent on traditional language courses;

increase in the number of languages offered for learning on the basis of a modular principle: not all aspects of the language are studied, but only certain ones, for example: computer English, Italian for exchange, Hungarian for reading, economic Russian, etc .;

- combination of intensive and extensive phases in language learning;

- $\quad$ expansion of receptive multilingualism;

- development of a teaching methodology a second (third) foreign language, taking into account the language experience of students and the possibility of intensifying the educational process;

optimization of the training system a foreign language teacher as a specialist in multilingualism for various levels of education, including adult education, and as an intermediary between the native and foreign language culture of students, as an expert who has deeply mastered the possibilities and technology of intercultural multilingual education;

opening courses in languages of the European (Eurasian) community at the language departments of universities;

- $\quad$ expanding the language training of specialists;

- $\quad$ expanding student exchange programs;

- expansion of training programs for teachers in the countries of the taught language;

- overcoming administrative barriers in creating multilingualism in schools and universities (in particular, opening groups with a small number of students in order to learn more languages);

development of new curricula for early language teaching, bilingual education and other forms with the specification of learning objectives, which, along with intercultural and communicative competencies, are also professional, mediatory in terms of language, etc.

It should be noted that the idea of linguistic rapprochement of peoples and cultures as means of harmonizing interpersonal and interethnic relations is by no means new: the artificial language of Esperanto was created by the Polish ophthalmologist L. Zamenhof (1859-1917) precisely as a means of establishing universal peace, a "bridge" between peoples and cultures, no matter how different they may be. True, the American linguist F. Folsom sarcastically notes that if it was easier for people to communicate, perhaps peace would reign sooner, but for people to stop killing each other once and for all, the unity of language is not enough. (Folsom, 1977). However, the vector of movement towards the specified goal has changed significantly (Figure 5). 


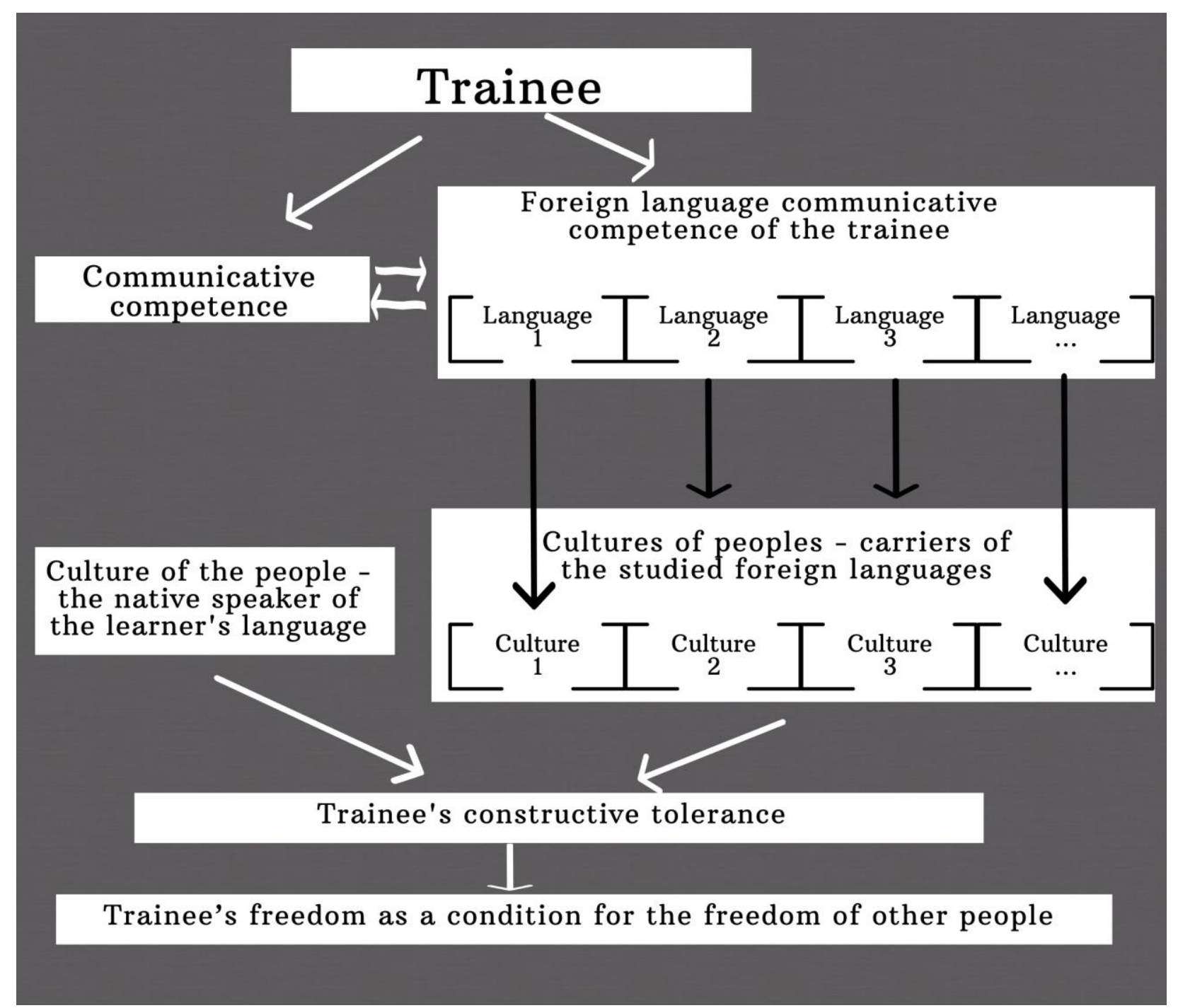

Fig. 5. Modern understanding of the dynamics of personality development in connection with the formation of foreign language communicative competence

However, the true acquisition of freedom, creativity and familiarization with the treasures of world culture, according to M. Heidegger are possible only if the individual refers to the need to comprehend his deepest essence to the fact that he is a thinking, that is, a comprehending being (Tepley, 2014).

It is "meaningful thinking that requires us not to cling one - sidedly to any one idea, to get off the habitual mental root along which we rush further and further (Tepley, 2014) the root of the global crisis, which in the field of education (in general) and teaching foreign languages (in particular) is manifested "... in the ever increasing subordination of quality to quantity, internal content and genius to technical means and techniques.

The larger and more varied the mass of material, the more difficult it is to process it and the more difficult it is to integrate it into a single stable harmony. The 
colossal nature inevitably leads to a deterioration in quality. At the same time, the emphasis on size means choosing the path of least resistance.

The turn to fundamental values in the practice of the formation of foreign language communicative competence predetermined transformations in the methodology of teaching foreign languages.

In this regard, the domestic education system today is focused on common European standards, which, along with the positive trends of modernization, is fraught with a real danger.

Thus, the absolutization of private methods of teaching foreign languages, illconsidered introduction of sometimes untested methods and technologies of Western pedagogy, without taking into account the needs of Kazakhstani society and the capabilities of the domestic education system, in our opinion, can cause harm rather than contribute to the solution of tasks defined by the President (On the State Program for the Functioning and Development of Languages, 1998) and Parliament (On Education,1999, 2001) of the Republic of Kazakhstan in the field of language teaching. In particular, it seems to us insufficiently justified to rely solely on communicative methods of teaching foreign languages and, accordingly, on communicative textbooks, the main disadvantage of which, in our opinion, is that it is the textbook, not the student, who determines the priorities in learning a foreign language.

A communication-oriented textbook is limited only to typical situations of communication, not taking into account the individuality of a specific national personality, due to its basic culture. On the other hand, behind the "shiny wrapper" sometimes conventionally new technologies are consigned to oblivion the traditionally strong sides of pedagogical science, one of which, in our opinion, is the orientation towards the formation of foreign language communicative competence. What is the complex of knowledge, skills and abilities that is traditionally called communicative competence in language teaching methods, as opposed to the concept of language competence?

The American linguist N. Chomsky first introduced the concept of linguistic competence into scientific use. It is a well-known fact that linguistic theories regularly changed the face of methodology in the 20th century. The notion of linguistic competence as a cognitive system that produces knowledge not antecedently present in the mind of the subject, e.g., knowledge of grammatical relations in response to certain stimuli is an important contribution to philosophical understanding of linguistics, and of cognitive psychology in general. This notion has not been as well received as it should have been, in part because of certain false things that have been said about it. In particular, it has been said that a grammar of a language, conceived as a theory of linguistic competence, is an idealization, and 
that speakers know the rules of the correct grammar of their language (Tienson, 1983). The theory of transformational generative grammar that appeared in the 60s of the last century was no exception: initially, methods of teaching the native language were created, which were based on the principle of generation, and then there were attempts to translate the principle of generation into methodological instructions for teaching a non-native language. For this study, it does not matter that attempts to transfer scientific linguistic theory into methodological practice have not been crowned with success (the XX century showed that such a transfer is, in principle, impossible, that the methodology of teaching languages should rather rely on their own research based on accounting "human factor"). However, the concepts of linguistic competence and "performance" took root in the methodology, since they provided a clear and definite distinction between two aspects in teaching (and learning) both one's own and a foreign language. The concept of linguistic and communicative competence have been filled with their own methodological content and today is one of the fundamental concepts underlying the construction of a particular method of teaching languages (Abdulrahman \& Abu-Ayyash, 2019).

In the works of foreign linguists of the 60s of the last century as for example the famous work of Leon A. Yakobovitz titled "Learning a foreign language. The experience of psycholinguistic analysis", much attention was paid to distinguishing between linguistic and communicative competence and the ways of their implementation. Despite the fact that the obviousness of the difference between the two concepts was recognized by all psychological theories of that time, there were no clearly developed psychological and methodological theories. Therefore, in relation to the speech act, the indistinctness (lack of prescription) of this division was found. It is interesting that the psychological research of this period was based on the basic idea that language competence is associated primarily with understanding and speaking - with the implementation of language competence. The work of L.A. Jacobovitz is interesting in that it is devoted to the definition of "performance" as an entity that manifests itself in the processes of understanding and speaking, which are in relation to each other in a hierarchical relationship. That is, it was assumed that initially there is an understanding of any grammatical form, and only then its use in speech (Jacobovits, 2008).

Without going into the history of the development and transformation of the concept under consideration, we note that even in the very first studies on this subject, the central point in relation to which differences were introduced was the speech act, in which the emphasis was on "performance" (in the modern understanding of communicative competence) as the implementation of language competence. 
Modern research in the field of methodology devoted to this theme continues the tradition of discussing the possibility of forming communicative competence only based on the conscious assimilation of the language system. This approach assumes that language teaching programs should be based on the study of the structure of the language, linguistic and stylistic norms and functional styles of speech in their organic unity, because in this unity the dialectical unity of the language system and the speech system is realized. As a basis for the formation of communicative competence, researchers identify linguistic competence knowledge inherent in the speaker - listener and allowing him to understand and generate statements. We share the opinion of D.I. Izarenkov, who proposes to understand the presence of practical knowledge of the language system under the language competence, and the theory in the field of linguistics under the linguistic competence (Izarenkov,1990). Thus, teaching the appropriate use of language means in speech involves combining a functional and systematic approach, taking into account the patterns of using language means in different functional styles.

This research is focused on considering linguistic, subject and pragmatic competences as the basic components of the communicative competence. These terms are delineated as follows:

linguistic competence is associated with the linguistic side of the organization of communicative units, it ensures the formation of the speaker's ability to build grammatically correct and meaningful statements;

subject competence is responsible for the content of statements, it ensures the acquisition of knowledge about the fragment of the world that is the subject of speech;

pragmatic competence reveals the speaker's communicative intentions, the conditions of communication, forming the speaker's ability to use utterances in certain speech acts, correlating them with intentions and conditions (communication situations)".

Pragmatic competence consists of knowledge that corresponds between communicative intentions and the statements that realize them; knowledge of the correspondences between the variable form of statements that realize the same intention, and the situational conditions of the speech act; the ability to realize a communicative intention by choosing a speech action in accordance with the requirements of the situation and the logic of the flow of the speech act (Izarenkov, 1990).

According to N.I. Gez communicative competence presupposes possession of linguistic (in our terms - language) competence, which includes: knowledge of information about the language; the ability to correlate language means with the tasks and conditions of communication, understanding the relationship between 
communicants; the ability to organize speech communication taking into account social norms of behavior and the communicative expediency of the statement $(\mathrm{Gez}, 1993)$.

The principle of the systems approach assumes that the acquisition of speech skills and abilities, the acquisition of speech are organically linked with the acquisition of language as a system and are based on the understanding of the lexical and grammatical meanings of linguistic units, awareness of their functional capabilities. The correct and appropriate choice and use of linguistic means in the process of speech presupposes that linguistic means and grammatical categories are studied not as a separate course in the framework of language learning, but as functioning in a certain sphere of communication, which sets a certain context of use.

Thus, mastering a foreign language communicative competence presupposes compliance with the following requirements: on the one hand, the student must be able to clothe his intentions in an adequate linguistic form and, accordingly, extract intentions, meaning from the statements of the interlocutor or the author (when reading, listening), building his communication tactics taking into account these intentions and communication circumstances. According to the authors of the Practical Methods of Teaching the Russian Language at the Initial Stage this approach involves the control of linguistic competence that is, checking the proficiency of the language system, correctness of speech. The first is determined using the well-known system "the number of errors for a certain length of text, utterance." Correctness of speech is also associated with taking into account errors, but not in the language system, but in the usus of word usage. In this case, the speech behavior of the trainees is assessed not on the "correct - incorrect" scale, but on the "norm - deviation" scale in comparison with the analogous speech behavior of a native speaker". Teaching the communicative competence of trainees presupposes the observance of the basic rule of communication - the reality (authenticity) of the communication conditions or the text presented for reading (listening), when there is an obligatory component of communication - the motive of the speech utterance, its authenticity, that is, the value outside the audience conditions.

On the other hand, the choice of communication tactics and forms of working with text should first of all take into account the set of requirements for the level of general communicative competence (in the native language), which must be achieved by law students within the framework of the general university training of a specialist. This circumstance determines the careful selection of communication skills and abilities that will form the basis for the organization of technology. The task is facilitated by the fact that some communication skills and abilities formed 
on the material of the native language can be transferred to a non-native language. Therefore, three groups of skills can be distinguished (Izarenkov, 1990) in the mechanism of foreign language speech:

speech skills in the native language, which should only be transferred to new language material and updated;

skills previously formed in the native language and which should receive correction when mastering a foreign language;

skills that must be formed a new.

Language teaching is a complex psychophysiological process that consists of two different methodological tasks' implementation, which can be formulated in a reduced form as follows:

- formation of language competence is a teaching the language system;

- formation of communicative competence is a teaching of language competence implementation through the formation of certain speech skills and abilities.

The question of mastering communicative competence during teaching the language of the specialty comes first, because in principle, assumes that the student has certain basic knowledge of the language (language competence). Textbooks on the language of a specialty, compiled abroad, as a rule, are guided by the average level of language proficiency (assuming the previous study of the basic course in the amount of 300 to 400 hours). Therefore, the main goal in teaching a language for special purposes at the postgraduate stage, we consider the formation of communicative competence, which involves defining as an urgent problem the methodology of developing students' ability to use language in different communication situations to achieve any, including professional goals. At the same time, it is necessary to take into account the fact that non-verbal means are widely used in communication and play an important role, that is, communicative competence involves mastering both verbal and non-verbal components.

Obviously, with this approach, the formation of linguistic competence turns out to be a subordinate task in relation to the goal of the formation of communicative competence, that is, the ability to solve communicative tasks using linguistic means in forms adequate to communication situations.

In the context of this research, foreign language communicative competence is considered in the aspect of a complicated complex of interpersonal interaction aimed at achieving professional goals. Therefore, it is necessary to make a certain reservation: postgraduates and adjuncts for whom the target language is not a native speaker, in principle, will not be able to achieve a certain level of communicative competence identical to the native speaker level. This limitation forces, firstly, to correlate the level of communicative competence with the level of language 
competence that corresponds to the goals and objectives of a certain stage of training. Secondly, it is necessary to take into account the level of general basic language training that was achieved by postgraduates and adjuncts before their inclusion in the training in the language of the specialty at the postgraduate stage.

In linguistics, the language of law is traditionally defined as a sublanguage. This concept can be defined as a system of artificially cultivated thematically organized specialized linguistic means, smaller in volume than the national language and occupying a subordinate position in it.

One of the features of sublanguages is the heterogeneity of the lexical units included in them, both in origin and in the categories to which they belong (narrow and attracted). Sublanguages are formed based on the literary language, but at the same time, being a sphere of scientific communication, they are characterized by a number of specific stylistic means (emphasized consistency of presentation, semantic accuracy, which does not allow for discrepancies). Unlike other sublanguages, the language of law (also called the language of legal literature) is characterized by transitional phenomena - the fusion of a scientific style with a business one, since the facts and circumstances set out in legal texts have an official orientation. In addition, the professional activity of a lawyer presupposes knowledge of not only scientific and official business styles, but also knowledge of the literary spoken language, since some activities (interrogations, speeches of defenders and prosecutors, work in the field of legal turnover, etc.) require use of clear and concise formulations, understandable and accessible to persons involved in the communication process. Therefore, in the study of language in jurisprudence, concepts such as the culture of speech and language norm are of great importance.

A language norm is a system of rules for pronunciation, use of words and use of grammatical means accepted in social speech practice. The language norm is stable and evolving at the same time. The stability of the language norm gives members of the language community the opportunity to understand each other and act together. Within the framework of traditional courses related to the study of linguistic norms, language is considered in the social context of the legal regulation of social relations: as a means of expressing legal information and as an attribute of a person acting in the legal sphere.

Recently, the issue of special training in the theory and practice of communication, the art of communication began to be raised by students themselves, who put forward the requirement to prepare them for real life in a communicative-speech relation (Flinders, 2015). It raises the interest of methodologists to subjects focused on the formation the corresponding skills, correlated with the broad concept of "culture of speech". 
The culture of speech in linguistics is the motivated use of linguistic material, the use of linguistic means that are optimal for achieving communicative tasks in a particular situation. The variety of communicative situations determines the need for a lawyer to master a wide range of functional styles. Therefore, in the written speech of lawyers, designed for preliminary reflection, the official-business style is preferable, while in the oral monologue, for example, in court, the means of the journalistic style are used. At the same time, the absence of a moment of preliminary reflection on the choice of linguistic means and emotional stress determine the appearance of features characteristic of oral speech.

The culture of speech includes normativity, that is, the ability to convey thoughts accurately and in accordance with the norms of the literary language; speech skill, which consists in intelligibility, consistency and appropriateness of speech, a variety of grammatical and syntactic structures, expressiveness. The culture of the written speech of a lawyer has its own characteristics, which consist in the motivated use of language means that meet the requirements of the official business style and the criminal procedure law, that is, adequately reflect the factual data established in the case. The speech of judges and lawyers operating within the legal profession is circumscribed to protect palpable functional interests, such as avoiding actual interference with the administration of justice, preserving the lawyer's obligations to maintain client confidences, or preventing misleading lawyer advertising or marketing. Public speaking is a necessary professional skill for all competent lawyers. Unfortunately, many do not understand the public speaking is different from just talking. Some lawyers think that they are good at public speaking because they can talk a lot and, regrettably, when they talk, they like the sound of what they hear. It does not occur to them that the audience may not be so delighted or interested.

Lawyers often speak before legislatures, city councils, planning commissions, and give talks to civic groups, business executives, or company employees - they even give media interviews on behalf of clients. Good public speaking is about the ability to communicate and connect with your audience. For lawyers this is even more important. People think that because you're a lawyer, you're automatically a fearless and brilliant public speaker. The expectation though, is one reason why it's more important for lawyers to have good speaking skills than it is for any other professionals. As a lawyer, it's critical that you know how to persuade a judge or tribunal, or speak to a group of clients, shareholders or conference participants (Haupt, 2016).

The culture of speech "presupposes possession of the forms and styles of the modern literary language in accordance with the goals and objectives of communication" (Skvortsov,1980). The culture of speech is understood as "the 
concrete implementation of linguistic properties and capabilities in the conditions of everyday and mass - oral and written communication" (Skvortsov, 1980). Rhetoric traditionally deals with the concrete implementation of linguistic properties and possibilities in speech, that is, linguistic requirements for speech are often combined with rhetorical ones. This can be traced in the system of communicative qualities of good speech (correctness, purity, accuracy, consistency, expressiveness, imagery, accessibility, efficiency, appropriateness of speech.

Therefore, as a rule, the issue of the specific implementation of the language system in the context of communication is discussed in terms of the "culture of speech communication", which is defined as "a highly developed ability to carry out communication in accordance with the norms that have historically developed in a given language community, taking into account the psychological mechanisms of influence on the addressee, and also using linguistic means and ways of implementing such communication, in order to achieve the greatest planned pragmatic result" (Faenova, 1991). According to M.O. Faenova the prerequisites for the culture of verbal communication include language / linguistic competence, communicative competence (the ability of an individual to generate socially acceptable statements), rhetorical competence (the ability, based on knowledge of psychological mechanisms of influence, to adequately use the expressive-emotional and / or logical means available in this language) (Faenova,1991). It is necessary to emphasize the difference in the methods of formation of speech competence: communicative competence is formed in a native speaker mainly in the process of socialization; rhetorical competence is the subject of special education.

In general, the question of systematization and classification of communicative and speech skills is of concern to both linguists and methodologists. It is important to note that communicative-speech skills are considered as the ability or readiness to perform a speech action because of acquired knowledge or imitation (Minaeva,1991), (Lvov,1988). In psycholinguistics and in the methodology of teaching languages based on it, the classification is based on the types of speech activity, namely: listening, speaking, reading and writing as the process of creating an utterance in writing (Lvov, 1988).

Accordingly, the following communication skills can be distinguished: ability to speak; ability to listen; ability to read; ability to write, create texts.

According to D.I. Izarenkov these types of speech activity constitute the concept of "communicative competence" which is understood as "a person's ability to communicate in one, several or all types of speech activity, which is a special personality trait acquired in the process of natural communication or specially organized training (Izarenkov, 1990). 
Thus, the concept of "communicative competence" implicitly includes linguistic competence, that is, it presupposes not only knowledge of the language, but also knowledge of information about the language. Also the ability to correlate language means with the tasks and conditions of communication, understanding the relationship between communicants and the ability to organize speech communication, taking into account social norms of behavior and the communicative expediency of the statement.

Within the framework of the activity approach, the main communication skills are formulated as follows: "a person should, in principle, have a number of skills. He must, firstly, be able to quickly and correctly navigate in the conditions of communication ..., secondly, be able to correctly plan his speech, correctly choose the content of the act of communication ... thirdly, find adequate means to convey this content ... fourthly, to be able to provide feedback. If any of the links in the act of communication is violated, then the speaker will not be able to achieve the expected results of communication - it will be ineffective" (Leontyev, 1974), i.e. achieving a state of adequate understanding between communicants is impossible.

The problem of comprehension and, in particular, adequate comprehension is one of the most pressing issues of epistemology. The need for communication based on understanding, i.e., according to P. Ricoeur comprehension of the meaning of signs transmitted by one consciousness and perceived by other consciousness's through their external expression (gestures, postures and ... speech) (Ricoeur, 2001) is an attributive property of an individual. In addition, since a person arises and exists only in interaction with other people and under their influence, communication based on clear mutual understanding is, on the one hand, the most important means of personal self-identification, and on the other, a condition for the stability of society. Certain aspects of the problem of understanding, interpretation and explanation are investigated by various sciences: logic and semiotics, psychology and history, etc. However, the experience of teaching foreign languages in connection with the theory of operations of understanding in their relationship with the interpretation of texts, that is, with hermeneutics, one of the most authoritative directions of Western philosophical thought, has remained unclaimed in the domestic teaching methodology until now.

The natural connection between hermeneutics and the theory of teaching foreign languages can be traced in the theoretical comprehension and creative use of the latter of certain techniques and means of cognizing the meaning of cultural phenomena (including teaching texts). In particular, philosophical hermeneutics summarized the achievements of ancient rhetoric, medieval theological hermeneutics, and philological hermeneutics of the Renaissance. Moreover, W. Dilthey (1833-1911), a German cultural historian and philosopher, a representative 
of the philosophy of life, the founder of understanding psychology and the school of the history of the spirit (history of the idea), expanded the logical-philosophical interpretation of hermeneutics and gave it the status of a methodology of the humanities. He considered understanding as "a process in which we comprehend the inner from sensually given signs", and hermeneutics as the art of interpreting written monuments, that is, the most universal manifestations of the human spirit (The Oxford dictionary of the Christian church, 2009).

The basic model of understanding that Gadamer finally arrives at in Truth and Method is the model of conversation. A conversation includes an exchange of views between interlocutors seeking an agreement on a dispute; hence, such exchange is never completely under the control of any interlocutor, but rather is determined by the subject of the dispute. Conversation always occurs in language, and in the same way Gadamer views understanding as always linguistically mediated. Since both conversation and understanding imply agreement, therefore Gadamer argues that any understanding includes something like a common language, albeit a common language, which is formed in the process of understanding itself. In this sense, all understanding, according to Gadamer, is interpretive, and since any interpretation involves the exchange between the familiar and the alien, any interpretation is also translational.

Gadamer's commitment to the linguistics of understanding also leads him to view understanding as a subject of conceptual articulation (Stanford Encyclopedia of Philosophy, 2018). This does not exclude the possibility of other modes of understanding but gives the advantage of language and conceptuality in hermeneutic experience. Indeed, Gadamer views language as not just a tool with which we can interact with the world, but as the very means of such interaction. We are "in" the world, being "on the tongue." However, this emphasis on the linguistics of understanding does not lead Gadamer to any form of linguistic relativism. Just as we are not inevitably held captive within the circle of our prejudices or the consequences of our history, we also are not held captive within the language. Language is something in which we can comprehend everything that can be comprehended, as well as that in which we meet ourselves and others. In this respect, language itself is understood as a dialogue or conversation. Like Wittgenstein and Davidson, Gadamer rejects the idea of a "private language" language always involves others, just as it always involves the world (Truth and Method, 1989).

As considered Swayne B.L. in dialectical hermeneutics, Gadamer argues that language is the universal horizon of hermeneutic experience; he also argues that the hermeneutic experience itself is universal. This is not only in the sense that the experience of understanding is familiar or ubiquitous (Barthold, 2010). The 
universality of hermeneutics stems from the existential claim to hermeneutics that Heidegger advanced in the 1920s and which Gadamer made the central idea of his own thinking. Hermeneutics concerns our basic way of being in the world, and understanding is thus the basic phenomenon in our existence. We cannot go back "for" understanding, since that would mean to assume that there was a way of intelligibility prior to understanding. Thus, hermeneutics turns out to be universal not only in relation to knowledge, be it in the "humanities" or elsewhere, but also in relation to all understanding and, in fact, philosophy itself.

Modern hermeneutics has formulated a number of general principles of text interpretation and teaching:

1) The principle of "whole and part", or "hermeneutic circle", understanding of the text is due to the understanding of the meaning of its individual parts, multilevel components (word forms, lexemes, syntaxemes, etc.), which helps the student to form a summary idea of the general meaning of the studied text. At the same time, the study of the meaning and significance of the structural units of the analyzed text presupposes the deployment in the student's mind of a preliminary projection of the meaning of the text as a whole.

2) the principle of understanding the meaning of the text based on the specific situation that gave rise to the statement, any unit of the text, be it a separate lexeme or phrase, is initially "built in" into the context. The text itself (from the Latin word "fabric", "connection") is a community of thoughts and words, linked by many links, a significant part of which is hidden, invisible. And the context, the consituation, is a much broader community, into which the text is woven, and is woven by ties that are already largely hidden. And the level of understanding of the text depends on how deeply and widely the subject of interpretive activity (the student) was able to grasp these connections, that is, to see in the text the expression of a complex and invisible reality, since, according to M.M.Bakhtin, every word (every sign) the text leads beyond its limits (Bakhtin, 1986). And the level of understanding of the text depends on how deeply and widely the subject of interpretive activity (the student) was able to grasp these connections, that is, to see in the text the expression of a complex and invisible reality, since, according to M.M. Bakhtin, every word (every sign) of the text leads out of its bounds (Bakhtin, 1986).

3 ) the principle of understanding the text based on itself, this principle implements Aristotle's thesis about language as the only way to express thinking. Studying the language in general and, above all, the written text was one of the main tasks. Early hermeneutic concepts established that all the thoughts and ideas of the author of the text can be found and cognized within the framework of the written work itself, in its "own" space. Thus, according to P. Ricoeur text is always something more than a linear sequence of phrases; it is a structured wholeness that 
can always be formed in several different ways. In this sense, the multiplicity of interpretations and even the conflict of interpretations are not a flaw or a defect, but a merit of understanding, which forms the essence of the interpretation; here we can talk about textual polysemy in the same way as they talk about lexical polysemy (Bakhtin, 1986).

4) the "productive" principle, etc. (Dilthey, 1992; Heidegger's ways, 1994; Dottori, 2012) the general scheme of understanding proposed by H. G. Gadamer is close to the dialectical materialist concept of cognition presented in Lenin's formula "from living contemplation to abstract thinking and from it to practice (Lenin, n.d.), since the process of understanding unfolds from "pre-understanding" (intuition) through "direct understanding", reading and familiarization with the text (sensory cognition) to "interpretation" (rational, theoretical knowledge) and - further "application" (practice, "practical" understanding). Thus, H.G. Gadamer brought understanding to a new stage - the stage of practical realization of the meaning of the text in a single situation of working with it, whether it be self-education, public education, etc.

It should be noted that the components of foreign language communicative competence, determined by the majority of modern domestic and foreign specialists in the field of teaching foreign languages, are quite consistently correlated with universal hermeneutic principles. In our opinion, the wide and consistent application of the latter for the development of methods for teaching foreign languages, selection and arrangement of teaching texts and tasks could significantly increase the effectiveness of the latter in solving the problem of forming a foreign language communicative competence of students at the postgraduate stage.

These prerequisites formed the basis for the development of a theoretical model for the formation of foreign language communicative competence of lawyers in the system of postgraduate education. (Figure 6, part 1).

So, the main prerequisites for the formation of the required competence are: the elaboration of the concept of competence as the main concept of the theory of the competence-based approach in pedagogy; interpretation of the essence of foreign language communicative competence as an organic unity and systemic totality of linguistic, subject and pragmatic competences; recognition of its correlation with universal hermeneutic principles; fullness of communicative competence with its own methodological content; specificity of the language of law as a sublanguage in linguistics. 


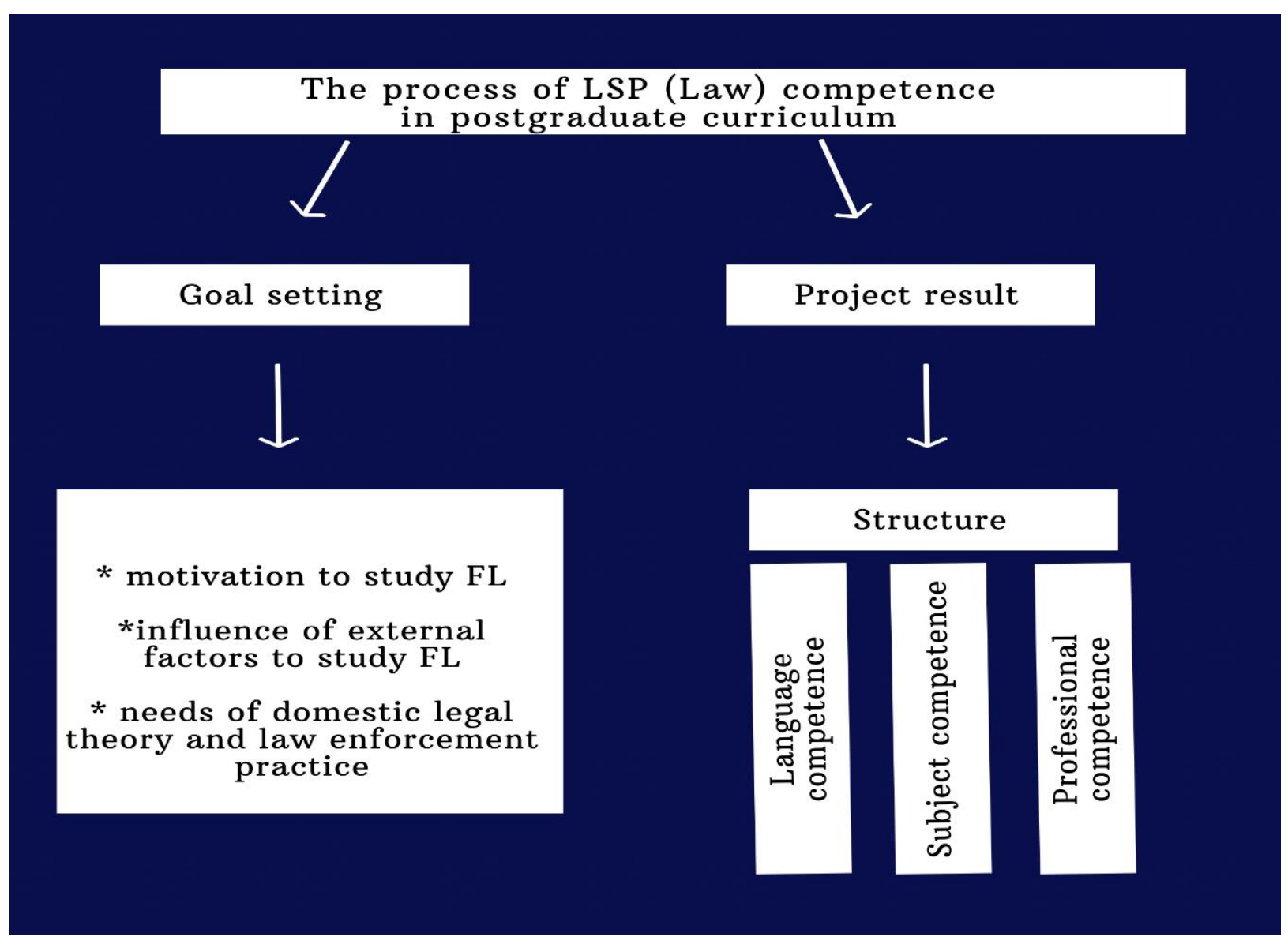

Fig. 6. Model of the foreign language communicative competence formation of a lawyer in the system of postgraduate education (part one) 


\subsection{METHODOLOGICAL FRAMEWORK IN FOREIGN LANGUAGE PROFICIENCY}

The process of integration into the world education system requires the primary formation of a specialist's readiness to carry out his professional activities through linguistic cooperation with representatives of other societies. Such cooperation is impracticable without competent proficiency in a professional foreign language of one's specialty. Kazakhstan's accession to the Bologna Convention will require universities to train competent specialists who speak a professional foreign language, who are able to constantly improve their experience, develop their individuality, including with the help of a foreign language, through involvement in professional discourse in this language.

The initial position in the development of this model was an orientation towards the result of training, which manifests itself in the formation of foreign language communicative competence in a master's degree in Law and the ability to communicate at the intercultural level, which leads to a multicomponent training. It includes both informational and procedural and organizational and motivational aspects as well.

In accordance with this, based on the theory of goal setting, in the process of forming the required competence, two main directions were identified: goal setting and goal implementation. The first of them is associated with setting of a goal and designing an expected result, the second one with converting a goal into a result Figure 6 (Part 2).

The design of the result is based on identifying and determining the criteria and indicators of the formation of foreign language communicative competence of lawyers, which include:

1) signs of linguistic competence (the ability to extract information from a coherent text of a legal orientation, build grammatically correct, meaningful syntactic whole assemblies from lexical structures in the form of a scientific text);

2) signs of subject competence (knowledge of the cumulative component of the native and foreign languages in the field of legal terminology, interpretation and practice of law enforcement, the ability to establish subject and interdisciplinary connections of the studied foreign language in the field of jurisprudence, to compare the conceptual fields of the legal vocabulary of the native and foreign languages);

3) signs of pragmatic competence (knowledge of the correspondences between communicative intentions (intentions) and statements that realize them; knowledge of the correspondences between the variable form of statements realizing the same intention and the situational conditions of a speech act; the ability 
to realize a communicative intention by choosing a speech action in accordance with the requirements situation and the logic of the speech act).

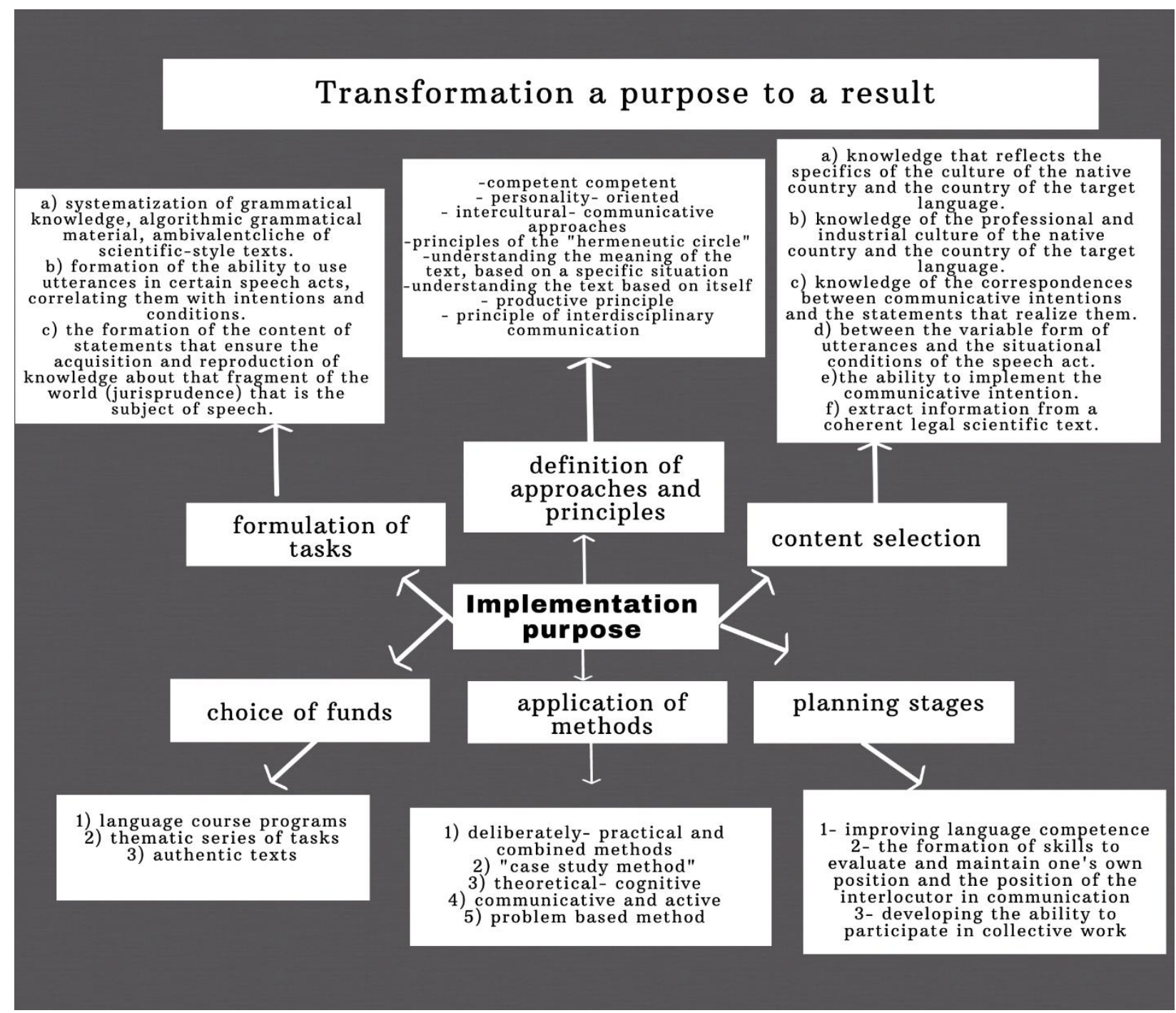

Fig.6. Model of the foreign language communicative competence formation of a lawyer in the system of postgraduate education (part two)

The indicated signs of foreign language communicative competence are grouped in accordance with its basic components (Figure 7), which includes:

1) language competence (ensures the formation of the ability to build grammatically correct and meaningful statements);

2) subject competence (provides knowledge about that fragment of the world that is the subject of speech);

3) pragmatic competence (reveals communicative intentions). 


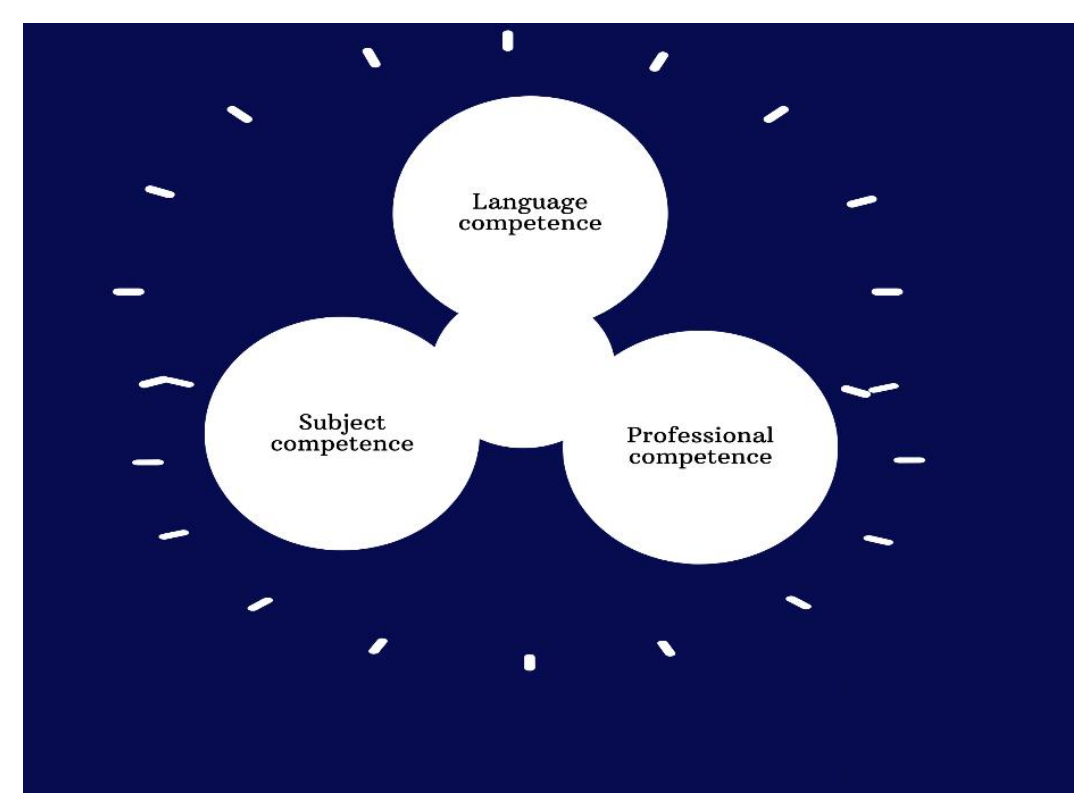

Fig. 7. The structure of foreign language communicative competence

It should be noted that in the scientific literature there is also the concept of linguistic competence, which, in our opinion, is identical to linguistic competence, since it includes knowledge of information about the language; ability to correlate linguistic means with the tasks and conditions of communication; understanding the relationship between communicants, etc. Therefore, we considered it appropriate to focus on three structural components, the features of which fully reveal the essence of the required competence. Based on these signs, we have identified the following criteria and indicators of the formation of a foreign language communicative competence:

I. Reconstructive variative:

1) ability to listen to information

2) ability to adequately respond to questions

3) skills of establishing contact with colleagues in a foreign language for organizing joint activities

4) ability to correctly present the listened or read information

5) ability to highlight the main idea of information when viewing the reading of scientific legal literature in a foreign language

6) skills in determining the meaning of a word based on knowledge of legal terminology.

II. Partial search

1) listen to scientific legal information, highlight and summarize 
2) be able to isolate the main information in the text

3) ability to highlight additional information in the message

4) be able to clarify the main points of the listened (read)

5) be able to analyze the content of a scientific legal text according to diagrams, figures, statistical data

6) be able to select sentences containing the main information

7) ability to highlight sentences containing additional information

8 ) be able to isolate keywords from the text

9) be able to extract information from a scientific legal article for compiling a message on a topic in a foreign language.

\section{Transformational}

1) ability to find confirmation of certain facts, events in a scientific and legal text

2) ability to find in a scientific and legal text a refutation of certain facts, events

3) be able to provide a rationale for the proposed title to a legal article

4) be able to correct the proposed plan for the article

5) be able to draw up a plan for the text in the form of affirmative or interrogative sentences

6) be able to draw up abstracts for a scientific article

7) be able to convey the content of a scientific article using reconstructed sentences

8) be able to compose your own message on the research topic, using information from several scientific legal articles

9) be able to lead a discussion on the topic of messages.

IV. Professionally significant qualities

1) development of skills of individual work

2) ability to work both individually and in a team

3) possession of formulas of politeness; the ability to self-esteem; the ability to act in non-standard situations

4) tolerance

5) ability to listen to others; behave politely, tactfully, diplomatically, restrained

6) ability to build your report, abstracts, message to the conference

7) skill of collecting material on a scientific topic

8) be able to argue. 
V. Motives of educational activity:

1) to become a highly qualified lawyer with knowledge of a foreign language

2) knowledge of a foreign language gives access to new professional legal information

3) desire to work with foreigners without an interpreter or abroad after graduating from the magistracy

4) I like to study a foreign language

5) successfully pass a qualifying examination in a foreign language

6) my future well-being will depend on the knowledge of a foreign language

7) desire to know a foreign language, just in case, will come in handy in life

8) successfully study and improve professional level

9) gain the approval of management and others.

The entire set of the above motives is the result of the objectification of a number of needs focused on:

a) object and purpose of the interaction,

b) interests of another person or society as a whole,

c) interests of the communicator himself and aimed at:

1) establishment of business ties for the implementation of joint activities,

2) communication with another person or a group of people to influence a person or a group of people

3) development of communication skills.

Taking into account the criteria and indicators of the formation of foreign language communicative competence among postgraduates, we have identified three levels of their actualization:

- poor level, when the subject does not have professional communication skills and does not have a conscious idea of them, or has, but does not have, the need to actualize them;

average level, when the subject has a conscious idea of the complex of skills and abilities, united by the concept of "professional communicative competence", but is not able to apply them in real activities;

- $\quad$ outstanding level, when the subject uses professional communication skills in all situations of scientific and business communication, when the named skills and abilities are adequate to the communication situation.

The transformation of a goal into a result involves a series of actions related to the formulation of objectives, the definition of approaches and principles, and the selection of content, the choice of means, the application of methods and the planning of stages. All actions are performed taking into account the specifics of the 
required competence, which we nominate as bi-communicative, the learner in the process of cognition carries out two-way communication: communicant $\leftrightarrow$ text, interlocuter $\leftrightarrow$ situation.

All actions are due to the nature of the basic components of a foreign language communicative competence. Therefore, the further presentation of the material in this subsection corresponds to their structural logic.

Modern global trends in the development of the world community, consideration of language as a translator of the global common human culture, expansion of international integration and intercultural interaction, strengthening of the culture-forming and cognitive-communicative functions of the language caused a radical revision of the methodology foreign language education (Tepley, 2014). In connection with the modern European culture-forming concept of teaching foreign languages, at present there is a departure from the absolutization of the communicative approach and purely communicative competence as the goal of learning and only speaks about the communicative orientation of the educational process. Intercultural (social) competence is put forward as a learning goal. The result of teaching a foreign language should be not only communicative competence, but also social and professional competence. The process of teaching a foreign language should turn into intercultural learning, into "teaching the understanding of someone else's", instilling in the student tolerance in socioeconomic, managerial, scientific theoretical and cultural innovations offered by the world community (World Declaration on Higher Education for the 21st Century: Approaches and Practical Measures, 1998). Such intercultural learning involves six sub-levels of language proficiency by CEFR (Common European Framework of Reference for Languages: Learning, Teaching, Assessment, Language Policy Unit, 2000). It is also desirable that the common reference points are presented in different ways for different purposes. For some purposes it will be appropriate to summarize the set of proposed Common Reference Levels in single holistic paragraphs, as shown in Table 1. Such a simple 'global' representation will make it easier to communicate the system to non-specialist users and will also provide teachers and curriculum planners with orientation points:

Table 1 - Common European levels of communicative competence (CEFR):

\begin{tabular}{|l|l|l|}
\hline & C2 & $\begin{array}{l}\text { Can understand with ease virtually everything heard or read. } \\
\text { Can summarize information from different spoken and } \\
\text { written sources, reconstructing arguments and accounts in a } \\
\text { coherent presentation. Can express him/herself } \\
\text { spontaneously, very fluently and precisely, differentiating } \\
\text { finer shades of meaning even in more complex situations. }\end{array}$ \\
Proficient
\end{tabular}




\begin{tabular}{|c|c|c|}
\hline User & $\mathrm{C} 1$ & $\begin{array}{l}\text { Can understand a wide range of demanding, longer texts, and } \\
\text { recognize implicit meaning. Can express him/herself fluently } \\
\text { and spontaneously without much obvious searching for } \\
\text { expressions. Can use language flexibly and effectively for } \\
\text { social, academic and professional purposes. Can produce } \\
\text { clear, well-structured, detailed text on complex subjects, } \\
\text { showing controlled use of organizational patterns, connectors } \\
\text { and cohesive devices. }\end{array}$ \\
\hline \multirow{2}{*}{$\begin{array}{l}\text { Independent } \\
\text { User }\end{array}$} & B2 & $\begin{array}{l}\text { Can understand the main ideas of complex text on both } \\
\text { concrete and abstract topics, including technical discussions } \\
\text { in his/her field of specialization. Can interact with a degree } \\
\text { of fluency and spontaneity that makes regular interaction } \\
\text { with native speakers quite possible without strain for either } \\
\text { party. Can produce clear, detailed text on a wide range of } \\
\text { subjects and explain a viewpoint on a topical issue giving the } \\
\text { advantages and disadvantages of various options. }\end{array}$ \\
\hline & B1 & $\begin{array}{l}\text { Can understand the main points of clear standard input on } \\
\text { familiar matters regularly encountered in work, school, } \\
\text { leisure, etc. Can deal with most situations likely to arise } \\
\text { whilst travelling in an area where the language is spoken. Can } \\
\text { produce simple connected text on topics which are familiar } \\
\text { or of personal interest. Can describe experiences and events, } \\
\text { dreams, hopes and ambitions and briefly give reasons and } \\
\text { explanations for opinions and plans. }\end{array}$ \\
\hline \multirow[t]{2}{*}{ Basic User } & $\mathrm{A} 2$ & $\begin{array}{l}\text { Can understand sentences and frequently used expressions } \\
\text { related to areas of most immediate relevance (e.g. very basic } \\
\text { personal and family information, shopping, local geography, } \\
\text { employment). Can communicate in simple and routine tasks } \\
\text { requiring a simple and direct exchange of information on } \\
\text { familiar and routine matters. Can describe in simple terms } \\
\text { aspects of his/her background, immediate environment and } \\
\text { matters in areas of immediate need. }\end{array}$ \\
\hline & A1 & $\begin{array}{l}\text { Can understand and use familiar everyday expressions and } \\
\text { very basic phrases aimed at the satisfaction of needs of a } \\
\text { concrete type. Can introduce him/herself and others and can } \\
\text { ask and answer questions about personal details such as } \\
\text { where he/she lives, people he/she knows and things he/she } \\
\text { has. Can interact in a simple way provided the other person } \\
\text { talks slowly and clearly and is prepared to help. }\end{array}$ \\
\hline
\end{tabular}


It is obvious that the majority of students in secondary schools achieve the level of initial proficiency in a foreign language (A1 and A2). Comparing to the requirements of the Standard Program in Foreign Languages for Higher Education Institutions of the Ministry of Internal Affairs of the Republic of Kazakhstan (Typical program in foreign languages for universities of the Ministry of Internal Affairs of the Republic of Kazakhstan, 2002) with the indicated levels of language proficiency, we conclude that upon graduation, the student's knowledge and skills must correspond to the B1 sub-level of the advanced proficiency level (see above).

Based on the provisions of the State Educational Standard of the Republic of Kazakhstan a master program student when studying a foreign language course is assigned to follow the tasks as getting acquainted with all types of reading (introductory, search, studying), literature in the specialty in a foreign language for receiving and transmitting scientific information, as well as literature of a sociopolitical nature. Knowledge and practical mastery of the grammatical minimum included into the university foreign language course is the basic grammatical phenomena of scientific style, taking into account its oral and written forms (Lvov, 1988). Thus, the state-defined level of knowledge and communication skills of the subject of postgraduate education should correspond to the B2 sublevel of advanced foreign language proficiency and, ideally, $\mathrm{C} 1$ fluency.

Considering the formulated tasks (Figure 8), namely: a) systematization of grammatical knowledge; algorithmic grammatical material; ambivalent clichés of scientific style texts; b) formation of the ability to use utterances in certain speech acts, correlating them with intentions and conditions; c) the formation of the content of statements that ensure the acquisition and reproduction of knowledge about that fragment of the world (jurisprudence) that is the subject of speech; we initially excluded the following tasks from the educational process:

- detailed study of morphological and grammatical categories of a foreign language (sublevels A1 and A2); (sublevel B1);

repetition of the legal vocabulary studied at the university stage

formation of initial communication skills (A1 and A2 sublevels) and professional interaction (B1 sublevel), since these tasks are fundamentally solved at school and university stages of education.

Therefore, the goal of postgraduate education is to optimize a foreign language communicative competence, components of which are the activation of linguistic, subject and pragmatic competences.

Linguistic competence is associated with the linguistic side of the organization of communicative units and is expressed in the ability to extract 
information from a coherent text of a legal orientation, to build grammatically correct, meaningful syntactic whole sets of lexical structures in the form of a scientific text. The task assumes the following:

- systematization of grammatical knowledge;

- algorithmization of grammatical material;

- ambivalent clichés of scientific-style texts (annotations, summaries, abstract).

Teaching, in principle is the art of the possible: sometimes the level of knowledge of a certain part of postgraduates does not correspond at all to the requirements presented at this stage of training, which makes it inevitable to refer to these categories. Secondly, we are talking not so much about teaching as about systematic, schematic and algorithmic ways of grammatical material in order to overcome the communicative barrier. Practice has shown that working with postgraduates, who have already completed a course of English grammar at the university, it is advisable to generalize and systematize the knowledge of students. In particular, to optimize their ability to build any complex verb form, which helps to easily recognize, and therefore, correctly translate syntactically a whole sentence any complexity that is satisfying and contributes to the entire learning process.

The scheme we propose is based on the main characteristics of the Russian predicate, in particular, in the indicative mood (time, type, voice). Using it, you can easily build a complex form of the verb, expressed by any combination of three characteristics (Figure 8).

The ability to recognize and generate verb-predicate forms, in turn, allows the postgraduate to perceive the supraphrasal unity not word by word, but holistically, an essential component of communication is in general and professional communication in particular. Structural coverage is taken into account when using schemes of this kind. In theoretical works on functional stylistics, a rather large place is occupied by the scientific style. As it is known, differentiation of styles is the result of the action of extralinguistic factors. The main extra linguistic styleforming factor according is a form of social consciousness with a corresponding type of activity and form of thought (Kozhina, 1966).

The main factor of the scientific style is conceptual and logical thinking. The deep features of the scientific style are associated primarily with the field of activity: the specificity of the scientific field of activity determines the specifics of communication between people in it and forms specific linguistic needs for the implementation of this communication (Kozhina, 1966). 


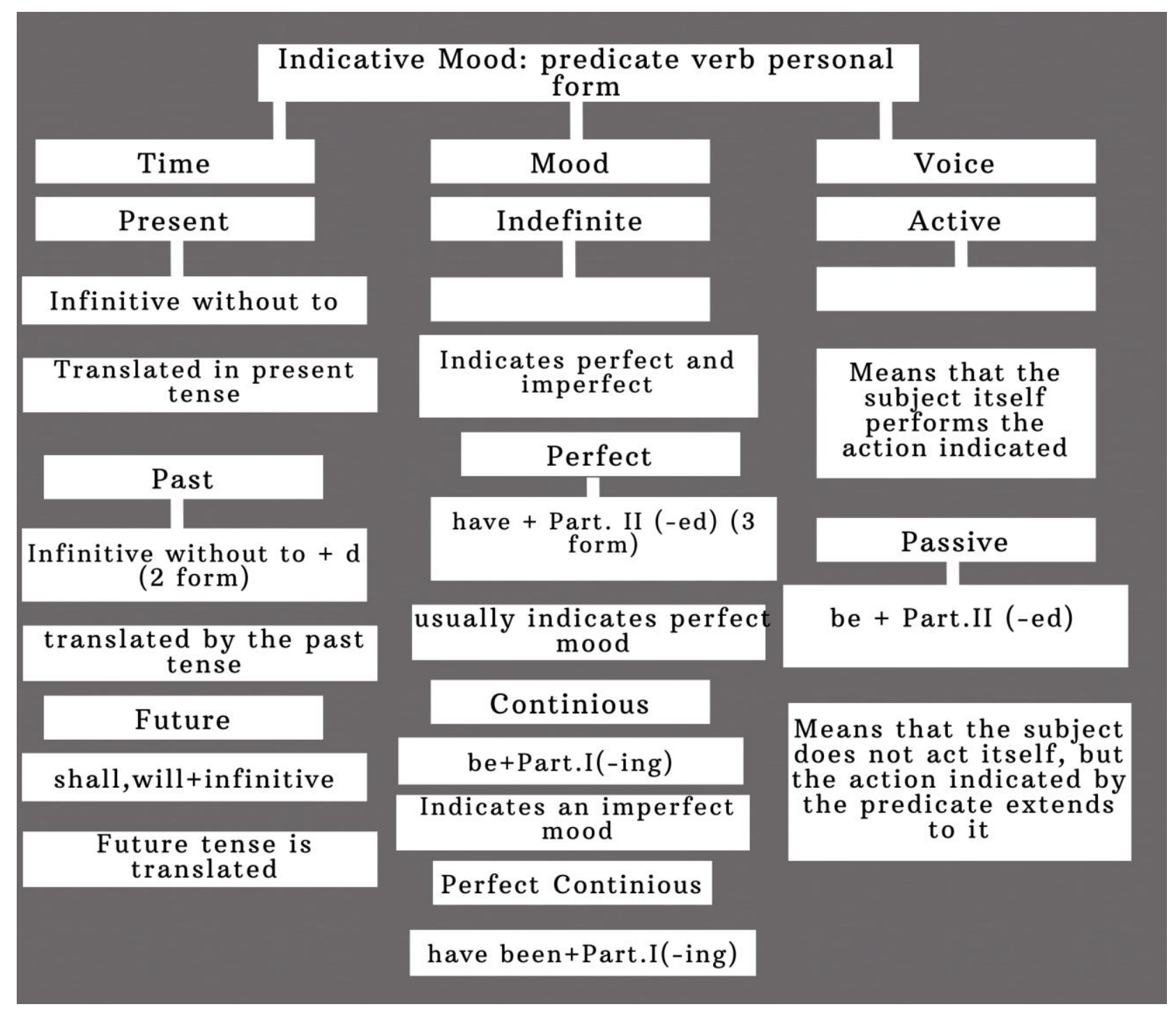

Fig. 8. Algorithm for the formation of the personal form of the predicate verb in the indicative mood in English

Both the producer and the recipient of speech in this area are specialists working in a certain scientific field, thus the addressees of speech with an equivalent conceptual and logical thinking. The study of the scientific style, the description of its features was carried out, as a rule, on the materials of written texts. Scientific communication can be both written and oral, where the main principle of scientific speech is the inclusion of a large number of terms that stand at the highest stage of the communication process.

Scientific speech is well known by a large number of terms. The terms are most capable of expressing conceptuality as one of the fundamental features of style. In teaching the scientific style, it is important to offer students multilateral linguistic assistance in writing a scientific work in English, based on modern achievements of 
linguistics, lexicography, teaching methods and the creation of expert teaching systems; to implement methods and techniques that encourage not to translate literally, but to tell and retell, not to reproduce what is written in the native language using the lexical means of the English language, but to present using ready-made models and expressions; not to overload the presentation with unfamiliar vocabulary equivalents, but to activate the existing stock of known English words and expressions.

The use of proper language is an essential element in writing scientific papers. Scientists communicate their findings or knowledge through language. If they do not use proper language, they will fail to convey the message. English has been used to write scientific papers for international journals or to participate in international conference. The language of science should be formal, straightforward, concise and brief. A scientist who uses complicated, metaphorical or ambiguous sentences may mislead the readers: accordingly, his message will not be properly understood by them (Suharno, 2017).

To write a good scientific paper, however, mastering certain grammatical rules (e.g. the use of tenses) is not enough, as a writing activity involves a number of other skills. For instance, when a scientist starts writing, he has to think of how his ideas are organized into sections and paragraphs. At the same time he has to choose appropriate words, phrases and grammatical structures. Using a series of long and complicated sentences throughout the whole paper will baffle the reader; on the other hand, using a stretch of short and simple sentences will be monotonous and boring to the reader. In short, a writer should take into account the factors mentioned above in writing in an appropriate style (Kennedy, 1984).

The English scientific style of speech is more compact, simple, active, relaxed, emotional and conversational, there is a distance between the author and the criteria for establishing the similarity of texts; conditions for the implementation of the communicative function of the language. The correct way to create a scientific article in English is not a translation, but a "story", not a reproduction of a "text (mother tongue)", but a "retelling", not a search for a literal equivalent, but the choice of an expression that is usually used to convey a given thought. No matter how carefully and conscientiously we tried to find the exact translation equivalent, say, for the mother tongue turnover, as shown by this study, it is safe to say that the result obtained will be unsuccessful, since in appropriate situations the study has revealed / shown in English is usually spoken. Therefore, it is often important to know the translation of a specific word, and to imagine what and how native speakers say in a given situation. So, in translations from the mother tongue language, long and numerous constructions with a genitive case - "nominalizations" are often reproduced, which have become the main distinguishing feature of all non- 
artistic styles of speech in mother tongue, including scientific, cf. variability of the chain with the preposition of. As a result, speech is replete with them, which is an unmistakable indicator of translation from mother tongue. Expression and presentation of your thoughts, like speech in general, is a special - active component of language proficiency, radically different from its passive component - reading and understanding. The ability to use clichés, expressions and certain equivalents constitute the student's scientific communication. To form the skill of interpreting a foreign language scientific text, authentic speech formulas, turns and expressions are used that are characteristic of the main structural stages of professional communication: Introduction $\rightarrow$ Data analysis $\rightarrow$ Discussion $\rightarrow$ Conclusion - in particular:

\section{Introduction}

\begin{tabular}{l|l|l|l} 
The & $\ldots$ chief / general & $\ldots$...im... & \\
\hline & $\begin{array}{l}\ldots \text { central / key / } \\
\text { ultimate }\end{array}$ & $\ldots$ goal... & \\
\hline & $\ldots$ main / particular & $\ldots$ purpose... & \\
\hline & $\ldots$ major / primary & $\ldots$ task & $\begin{array}{l}\text {.. of this paper / study is to } \\
\text { investigate / establish P. }\end{array}$ \\
\hline
\end{tabular}

One of the main/ principal objectives

\begin{tabular}{|c|c|c|}
\hline The & ... burden & \\
\hline The & ... subject (matter) & $\begin{array}{l}\ldots \text { of our paper / study / analysis / research / } \\
\text { discussion is P. }\end{array}$ \\
\hline \multirow{4}{*}{\multicolumn{2}{|c|}{ The present paper / investigation }} & ...goes into / focuses on / deals with P. \\
\hline & & $\begin{array}{l}\ldots \text { is devoted to the questions (problems) of } \\
\text { P. }\end{array}$ \\
\hline & & $\begin{array}{l}\ldots \text { undertakes to survey / identify the } \\
\text { structure of P. }\end{array}$ \\
\hline & & $\begin{array}{l}\ldots \text { considers what factors } / \text { processes } \\
\text { influence } P \text {. }\end{array}$ \\
\hline \multirow{4}{*}{\multicolumn{2}{|c|}{ In this article / section }} & $\begin{array}{l}\text {.. I aim to determine / I attempt to explain } \\
\text { the mechanisms of P. }\end{array}$ \\
\hline & & $\begin{array}{l}\ldots \text { I am intended to give / show / develop / } \\
\text { provide / record P. }\end{array}$ \\
\hline & & $\begin{array}{l}\text {... I examine the nature / characteristics / } \\
\text { features / functions of } P \text {. }\end{array}$ \\
\hline & & $\begin{array}{l}\text {... I (will) concentrate on / argue that / review } \\
\text { P. }\end{array}$ \\
\hline
\end{tabular}




\section{Data analysis}

\begin{tabular}{l|l} 
Our description & $\ldots$ rests on evidence obtained experimentally. \\
\hline Our analysis & $\ldots$ rest on evidence available from biology. \\
\hline This research / study / claim & $\ldots$ rests on findings/materials of P. \\
\hline This investigation & $\ldots$ draws on observations of P. \\
\hline We limit the analysis to & $\begin{array}{l}\text {... two phenomena (sample cases / substances), P } \\
\text { and Q. }\end{array}$
\end{tabular}

\section{Discussion}

What do these findings / observations / results / numbers / figures mean?

These results / observations $\quad$... need careful explanation / require a careful
study.

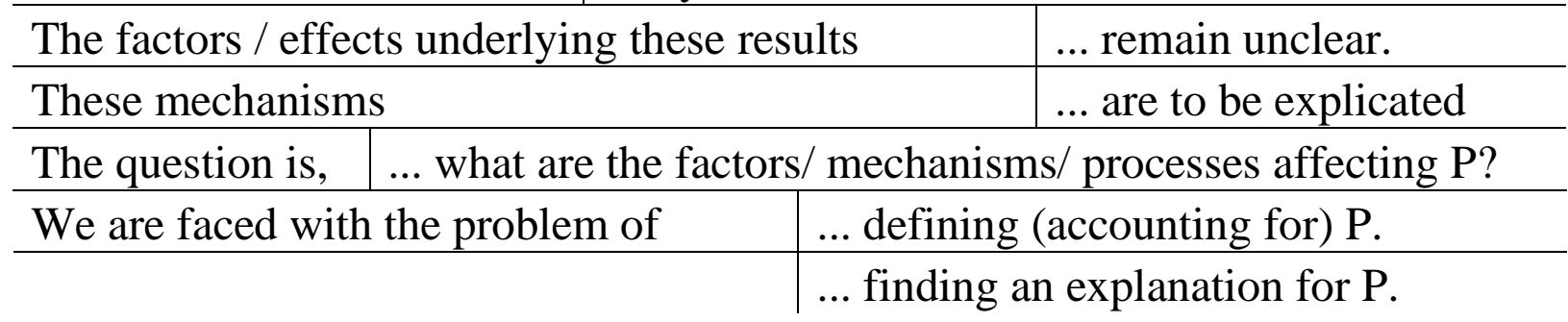

\section{Conclusion}

\begin{tabular}{l|l}
$\begin{array}{l}\text { The conclusions of the } \\
\text { study }\end{array}$ & $\ldots$ reveal that P / will help illuminate/ explain P. \\
\hline We have succeeded in & $\ldots$ actually showing (that) $\mathrm{P}$. \\
\hline Our results & $\ldots$ highlight the potential of the technique. \\
\hline & $\ldots$ could lead to (better measurements of) $\mathrm{P}$. \\
\hline & $\ldots$ can be directly applied to the process of P. \\
\hline Our technique & $\begin{array}{l}\text {... can be applied directly / gradually / partially / in } \\
\text { addition to/ in combination with P. }\end{array}$ \\
\hline Our method & $\ldots$ can be developed much further/ is easier to apply. \\
\hline The merits of the method & $\ldots$ are in P.
\end{tabular}

The next component of postgraduate education is subject competence, which forms the content of statements, ensuring the acquisition and reproduction of knowledge about that fragment of the world (jurisprudence) that is the subject of a master's student's speech, which determines:

a) establishment of subject and interdisciplinary links of the studied foreign language in the field of jurisprudence; 
b) comparative study of the conceptual fields of the legal vocabulary of the native and foreign languages;

c) associated, more precisely conditioned by this, the development of the cumulative component of the native and foreign languages in the field of legal terminology, interpretation and practice of law enforcement.

Because the specificity of communication between people of different professions is determined, first, by the objective world, which is its material basis, it is logical to establish those concepts on which the process of teaching a foreign language at the postgraduate stage can be based. The selection is made from a number of concepts that serve the professional communication of lawyers and are recorded in the legal conceptual apparatus. The selection procedure is based on the method of comparing subject concepts that function in the speech of a specialist and the concepts available to students in terms of volume, diversity, and degree of complexity.

\section{Conclusion}

The professionally significant knowledge of trainees is based on the set of concepts. We designated in Section 1 the term "subject competence", providing them with the opportunity to navigate the content of professionally oriented teaching of a foreign language in the chosen subject area. Thus, the main selection criterion is the correspondence of concepts from the conceptual apparatus of lawyers to the level of subject competence of students. The trainees are postgraduates, applicants and adjuncts with a special legal education and, as a result, level of subject competence of students in the period under review is high, conceptual apparatus is defined, volume of background knowledge is not limited. Based on the results of the analysis of the lawyers' conceptual apparatus and the analysis of trainees - postgraduates' subject competence, we can conclude that subject competence reflects the knowledge of communicants in a certain special field. For example criminal law, criminal procedure, civil law, management of public security services, organization of crime investigation, knowledge about the country of the target language, necessary for the adequate solution of professional tasks (comparative analysis of sources of law, legal concepts, competence of legal professions) and subject competence as a unity of interest and awareness in the content of communication. We believe that it is necessary to focus on the substantive and content side of communication. Since, in essence, the professionally significant knowledge of postgraduates, adjuncts and applicants is nothing more than a reflection of the professional and production culture, which in our work appears in the form of professional universals and concepts with sociocultural 
specifics, it is natural to single out the relevant aspects in the professionally significant knowledge of students:

1) knowledge of professional universals;

2) knowledge that reflects the specifics of the culture of the native country;

3) knowledge that reflects the specifics of the professional and industrial culture of the native country;

4) knowledge that reflects the specifics of the culture of the country of the target language;

5) knowledge in the field of professional and industrial culture of the country of the target language.

The structure of the subject competence of the student is shown in Figure 9.

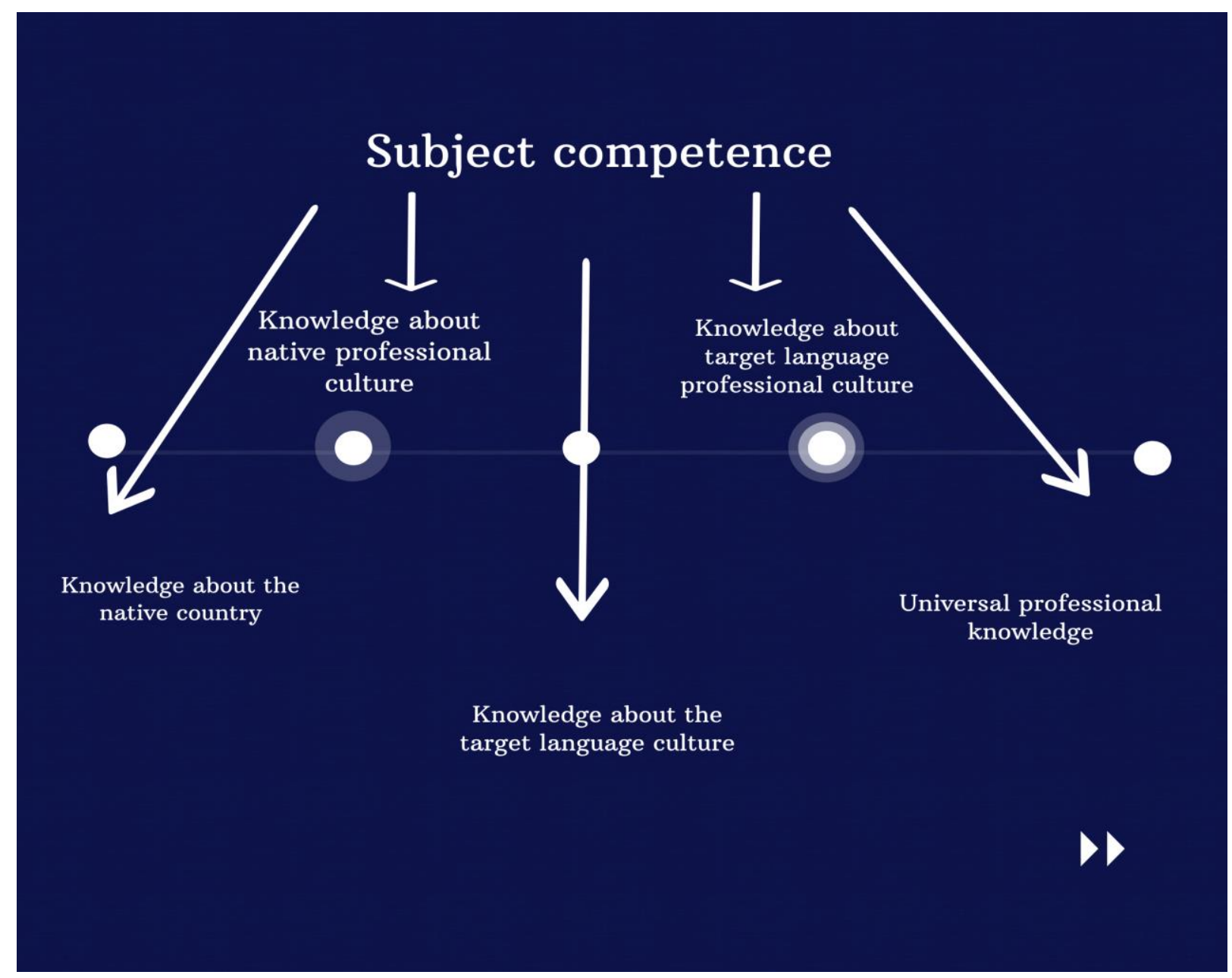

Fig. 9. Subject competence structure

It is worth noting what we mean by qualification - competencies that are directly related to a specialty or academic discipline. Therefore, it is quite reasonable to consider subject-specific competences. 
Subject-specific competencies are what individuals need to know, understand, and be able to do in relation to a particular discipline (SJUR Bergan, 2007).

In higher education, the term "subject-specific competencies" is usually used to refer to those who specialize in a given discipline, however, it can also describe competencies achieved in the same discipline by non-specialists. For example, if a law curriculum requires a competency in foreign language proficiency, this competence should be defined in the curriculum, for example, as "English for Lawyers". For example, a lawyer may be required to be able to speak and write in their native language, and at least one foreign language at some level. Calling such a competence subject-specific or universal is a matter of preferences and the dividing line will be extremely arbitrary.

The following principle can be proposed: to consider a competence as universal if its presence is implied by a qualification and the organized training program does not contain a course designed to develop this competence. If there is such a course, then the competence should be considered subject-specific. The program of study at the postgraduate stage establishes a certain level of proficiency in a foreign language as a requirement and provides for the study, for example, of English language for special purposes, in order for master students to acquire the appropriate competence, and in this case, we consider it as subject-specific. To a certain extent, subject-specific competencies are a special case of applying broader, universal competences to a particular discipline, and they are a collection of methodologies, mindsets and knowledge specific to that discipline.

Let us consider to what extent and of what nature professionally significant knowledge is given to students in the magistracy.

Analysis of the curriculum for the preparation of postgraduates in the specialty "Law enforcement", structured according to the State Educational Standards of the Republic of Kazakhstan, made it possible to establish the distribution of major disciplines, systematized on the basis of consultations with specialists.

The disciplines studied at this stage, by their nature, relate to narrowly professional (table 2), which, in fact, means studying not "in breadth", but "in depth". In particular, the course "Managing the activities of public security services" involves the formation of the master student's skill "to make managerial decisions on the organization of activities of units and public security services" (Public Security Management. Syllabus, 2016). The purpose of teaching the discipline is to expand and deepen the knowledge of postgraduates about the patterns of management activities, to form an idea of the role of public security services in protecting public order. The course "Criminal Law" is part of the fundamental 
training of postgraduates. The scope and depth of the knowledge presented in this course is great, and its complexity is potentially increased. In addition, as it turned out from consultations with teachers of these disciplines, information is given using concepts-universals (conceptual fields) (Problems of complicity in the criminal legislation of the Republic of Kazakhstan, 2016).

As a result, it seems that at the subject level the content of training should be based on a set of criminal, civil, procedural concepts with detail and problematic deepening. The problem of coordination of knowledge is closely related to the issue of concentration of interdisciplinary ties at different stages of training. The sequence of the appearance of certain concepts in the learning process is reflect retrospective, parallel, perspective stages.

Table 2. Distribution of major disciplines:

\begin{tabular}{|l|l|}
\hline$\#$ & \multicolumn{1}{|c|}{ Discipline name } \\
\hline I & BASIC DISCIPLINES \\
\hline $\mathbf{1}$ & History of philosophy and science \\
\hline $\mathbf{2}$ & Foreign language \\
\hline $\mathbf{3}$ & Management \\
\hline $\mathbf{4}$ & Psychology \\
\hline $\mathbf{5}$ & Pedagogy \\
\hline & Optional component \\
\hline $\mathbf{I I}$ & PROFILE DISCIPLINES \\
\hline & Required component \\
\hline $\mathbf{1}$ & Organization of operational-search activities \\
\hline $\mathbf{2}$ & Organization of investigation of crimes \\
\hline $\mathbf{3}$ & Public safety management \\
\hline $\mathbf{4}$ & Modern problems of the general theory of law \\
\hline $\mathbf{5}$ & Theory and practice of the application of criminal legislation \\
\hline $\mathbf{6}$ & Theory and practice of the application of criminal procedure legislation \\
\hline $\mathbf{I I I}$ & PROFILE DISCIPLINES \\
\hline & Compulsory component \\
\hline $\mathbf{1}$ & Organization of operative and search activities \\
\hline $\mathbf{2}$ & Organization of investigation of crimes \\
\hline $\mathbf{3}$ & Public safety management \\
\hline $\mathbf{4}$ & Modern problems of the general theory of law \\
\hline $\mathbf{5}$ & Theory and practice of the application of criminal \\
\hline $\mathbf{6}$ & Theory and practice of the application of criminal procedure legislation \\
\hline $\mathbf{7}$ & Management in law enforcement \\
\hline & \\
\hline
\end{tabular}




\begin{tabular}{|c|l|}
\hline $\mathbf{8}$ & Law enforcement activities to ensure human rights \\
\hline $\mathbf{9}$ & Information activity of law enforcement agencies \\
\hline & Optional component \\
\hline $\mathbf{I V}$ & RESEARCH PROJECT \\
\hline $\mathbf{V}$ & PRACTICE \\
\hline & $-\quad$ Pedagogical \\
\hline & $-\quad$ Professional \\
\hline $\mathbf{V I}$ & FINAL STATE CERTIFICATION \\
\hline $\mathbf{1}$ & Comprehensive exam \\
\hline $\mathbf{2}$ & Master's thesis defense \\
\hline
\end{tabular}

Different types of interdisciplinary connections are needed. It seems that the interdisciplinary connections established at the level of professionally significant concepts should be enriched and strengthened with new factual material, which can be obtained mainly by means of a foreign language. In this case, interdisciplinary communications will not function one-sidedly, only in the interests of teaching English at the postgraduate stage, but will also provide feedback, i.e. contribute to the expansion of the factual base of students, providing them with information that he can use both in the study of professionally oriented disciplines and in scientific research. Moreover, ultimately, the development and consolidation of the concepts used in the emerging system of knowledge.

Any professionally directed human activity is reflected in the language, the peculiarity of which is manifested, first, at the lexical level. The development of civilization is also characterized by far-reaching specialization in all areas of human activity. Each social or professional environment creates its own language, consisting of specialized terminology and established phraseology, often including grammatical forms that differ from the norm. The nature of these special languages, as well as their impact on the common language are obvious. Since these communities stand out against the background of the social life of society as a whole their language expresses not only what is associated with this or that type of activity, but also things and actions from life in general, which often receive new names (Balli, 2003). Each branch of knowledge has specific words and phrases inherent in it, terms denoting scientific concepts about objects, phenomena, signs, which together with other concepts of a particular branch of science or technology constitute one semantic system. The terms are formed in order to free speech from an ambiguous understanding of the phenomena of reality. Professional communication requires clarity, concreteness and brevity, which is largely made possible by the widespread use of terms. When translating terms that carry key information, attention should be paid to the terms and their foreign language 
equivalents. The English language has a developed terminological system for denoting legal realities. Therefore, for the designation of legal professions, there are terms many of which are synonymous: lawyer, law agent, jurist, jurisprudent, solicitor, advocate, attorney, legist, legalist, barrister, counsel, counselor, legal expert, man of law.

Legal professionals in today's world must be comparative and broad-minded to conduct any research, study or work in any other way in multicultural or multiple legal systems. Legal professionals need to know that they must free themselves from their own doctrinal and legal biases and free themselves from their own cultural context in order to find neutral concepts to describe problems (Zweigert \& Kotz, 1998). The legal professional must apply a combination of formal legal knowledge and formal legal skills in a more creative way (IBA International Principles on Conduct for the Legal Profession, 2011) whereas broker may have insider knowledge of the system but not proper formal skills and knowledge to advice client. The legal profession refers to the whole of occupational roles purposely oriented towards the administration and maintenance of the legal system, including judges, lawyers, counsellors, as well as experts of legal education and scholarship.

The most general term is lawyer, which translates as lawyer, advocate. A wide semantic field covers the terms: jurisprudent, legist, legalist, legal expert, man of law which depending on the specific situation are also translated as a lawyer, advocate. Law agent is used to refer to any lawyer, with the exception of a lawyer and translated as attorney, solicitor. The complex terminological system reflects the gradation within the lawyer's profession: advocate is the most general term in this series; solicitor is a lawyer who advises a client, prepares cases, but has the right to appear only in lower courts, an attorney; barrister is a lawyer who has the right to appear in higher courts, it is for him that solicitor prepares cases. Counsel and counselor are also used in the sense of a lawyer, but refer to a lawyer giving advice, a legal adviser. The closest synonym for these two terms is barrister. The term attorney has several meanings, including an attorney, but it is primarily used to refer to an attorney.

The obvious lack of legal terminology in the Russian language has forced lawyers to copy many names of legal professions. Therefore, in the Kazakh or Russian languages, the terms solicitor, barrister, attorney appeared, which, in turn, need interpretation for non-specialists (Zhetpisbayeva \& Uteubayeva, 2012). 
Below are the following examples of a comparative study of conceptual fields' maps of the legal vocabulary concerning native and foreign languages, which we use in practical classes in English in the magistracy (Figures 10 and 11).

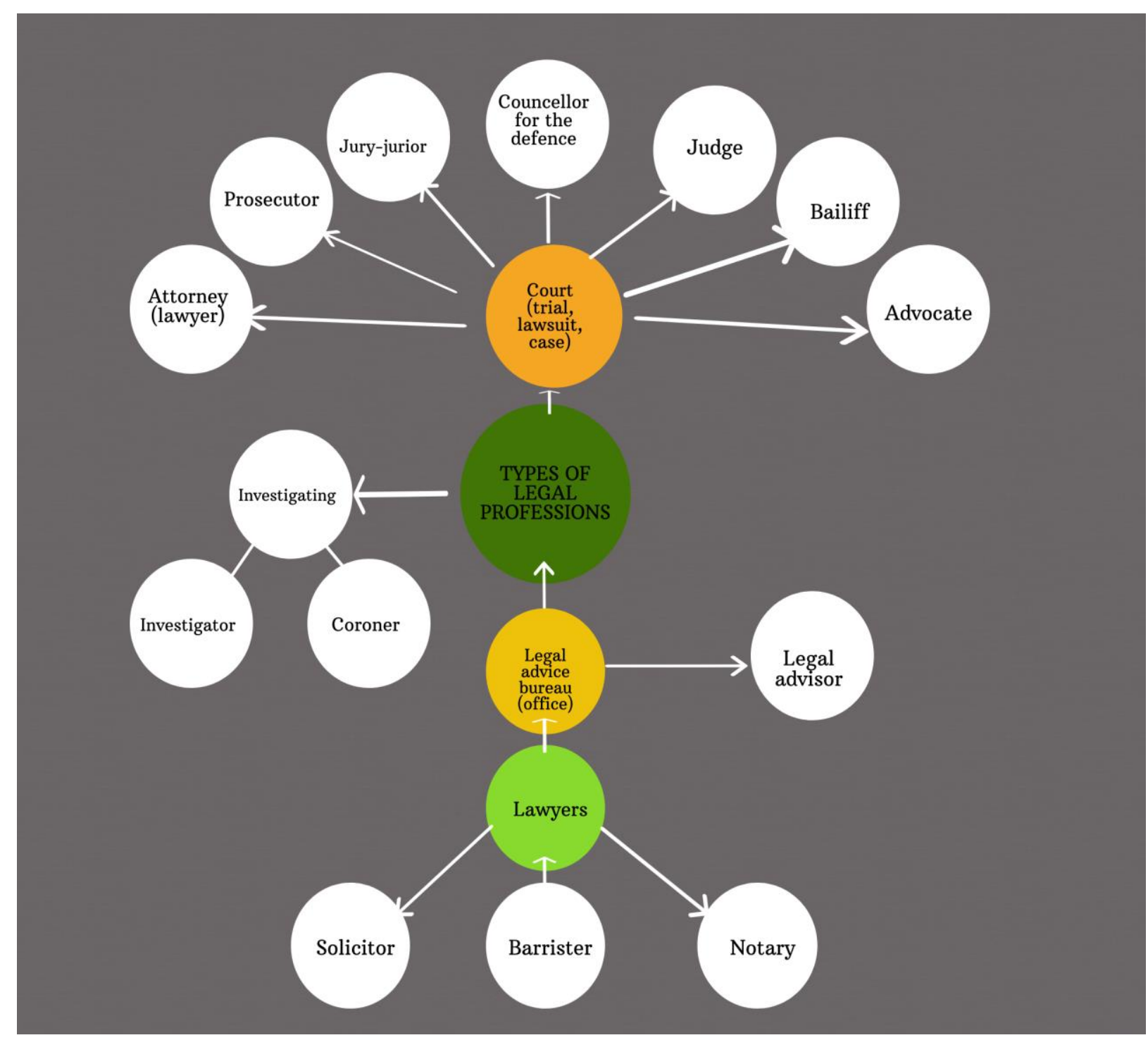

Fig. 10. Types of legal professions 


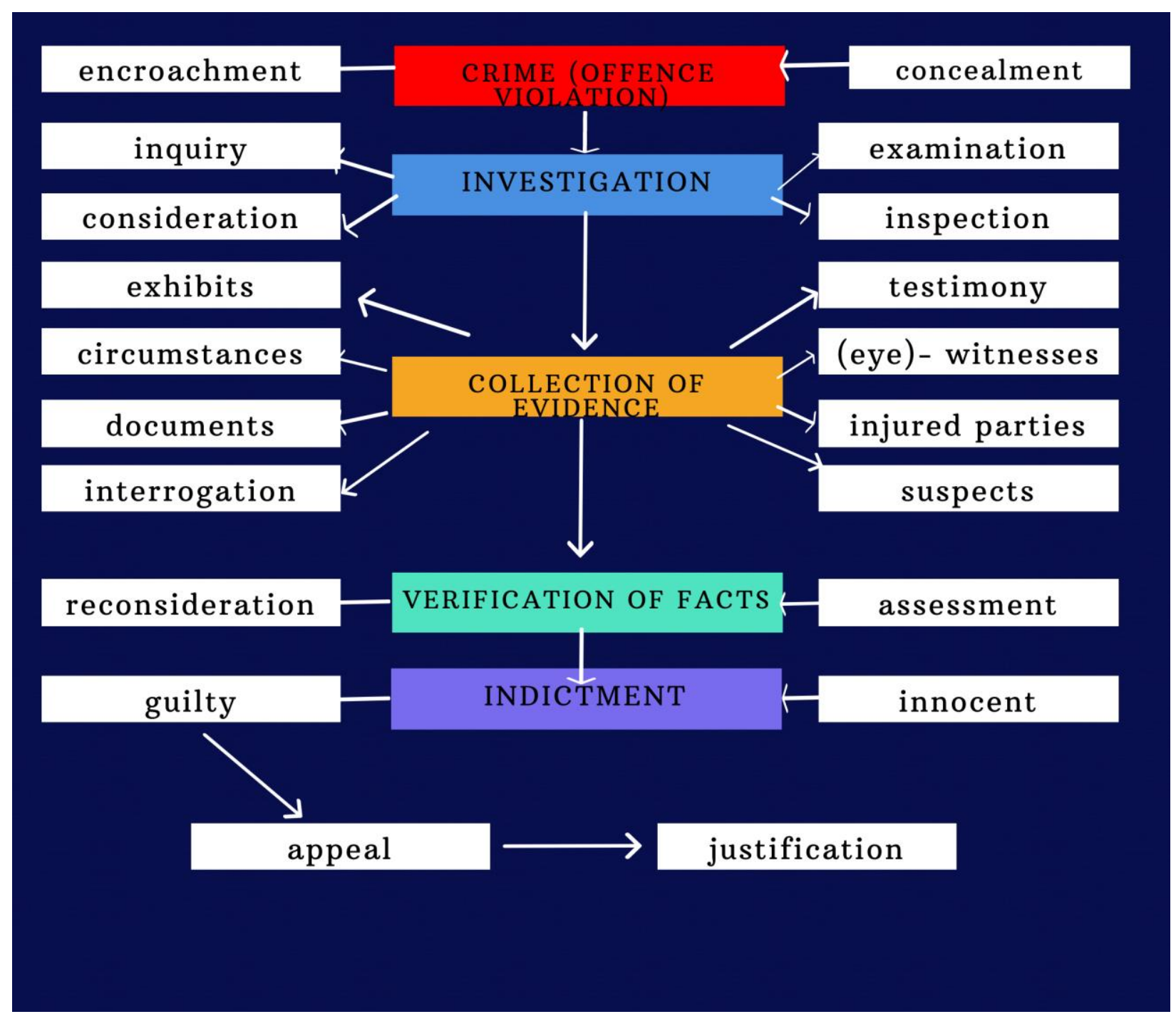

Fig. 11. Stages of criminal investigation

The next component of a foreign language communicative competence of a lawyer is pragmatic competence, which reveals the communicative intentions of the speaker, conditions of communication forming the communicants' ability to use statements in certain speech acts, correlating them with intentions and conditions (communication situations), namely:

a) knowledge of the conformity between communicative intentions and the statements that realize them;

б) knowledge of the conformity between the variable form of statements that realize the same intention, and the situational conditions of the speech act;

в) ability to implement a communicative intention by choosing a speech action in accordance with the requirements of the situation and the logic of the speech act. 
Thus, based on the requirements of the State Educational Standards of the Republic of Kazakhstan and our views about the goals and objectives of teaching a foreign language at the postgraduate stage, we can define the proposed concept as bi-communicative: a student in the process of cognition carries out bi-directional communication: interlocuter $\leftrightarrow$ text, interlocuter $\leftrightarrow$ situation (Figure 12).

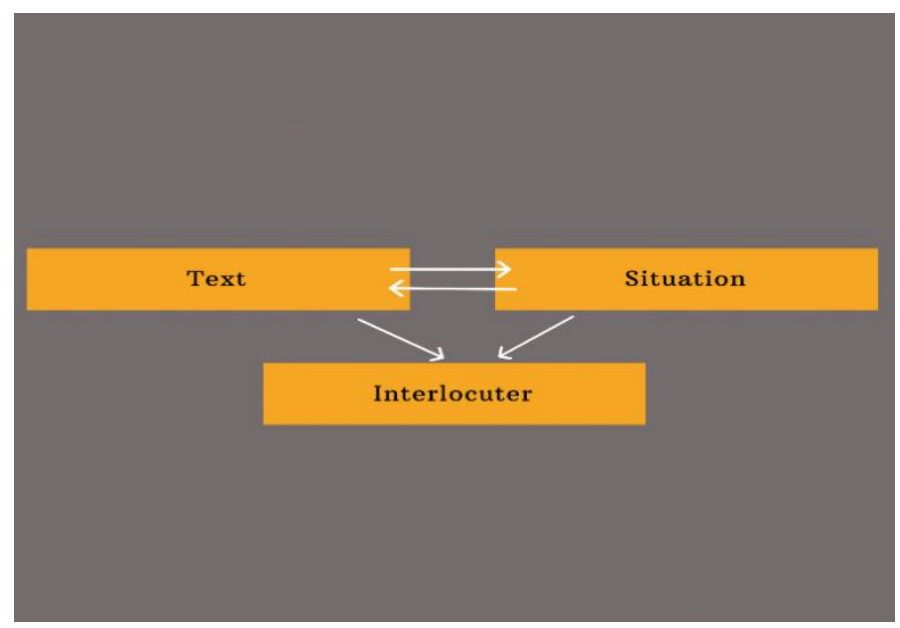

Fig. 12. Interaction of participants in the learning process

Moreover, at the heart of the proposed method the understanding of a text is due to the understanding of the meaning of its individual parts, multi-level components (word forms, lexemes, syntaxemes, etc.), which help students to form a summary idea of the general meaning of the text being studied. At the same time, the study of the meaning and significance of the structural units of the analyzed text presupposes the deployment in the student's mind of a preliminary projection of the meaning of the text as a whole.

On the other hand, the meaning of the text can be adequately understood from the situation that gave rise to the statement: any unit of the text be it a separate lexeme or phrase is initially "built in" into the context. According to M.M. Bakhtin behind each text there is a system of language. In the text, it corresponds to everything that is repeated and reproduced, everything that can be given outside the given text (given). On the other hand, each text is something individual, unique and inimitable, and this is the whole point of it (its intention, for which it was created). This moment (pole) is inherent in the text itself, but it is revealed only in a situation and in a chain of texts (in speech communication of a given area)" (Bakhtin, 1976).

The choice of language means for the experimental course of the specialty language to be quite difficult. The specificity of objects (topics) of communication to be discussed lies in the fact that situations are taken from real professional reality and their description, in principle, cannot be "captured" by a closed list of lexical minimums. 
It is impossible to ignore the fact that the general literary language is not assigned to any specific sphere of student's activity. The most frequent vocabulary in colloquial speech is general literary. The same can be said about nonterminological scientific vocabulary, and about other linguistic means. So, the main stylistic layers of vocabulary are not distributed in accordance with the functional types of speech, in various texts attributed to certain types of speech, in many cases it is impossible to find lexical "style indicators" (Shmelev, 1977). Functional styles are characterized, first, by "a special, specific organization of general linguistic means, conditioned by its functional orientation, and only then by a certain special set of linguistic means" (Kozhin, 1982). In other words, literary speech is notable for its universality in the sphere of functioning. On the basis of this speech, scientific essays, journalistic works, scientific and technical works are created, this speech is the basis of fiction, journalism, business writing (Order of the Ministry of Internal Affairs of the Republic of Kazakhstan "On approval of the Program for the development of departmental education of the Ministry of Internal Affairs of the Republic of Kazakhstan for 2017-2020, 2016).

In addition, the peculiarity of speech in the professional field of jurisprudence is that it combines prepared and spontaneous forms, often clichéd, which ideally are in the student's memory (and should be updated in the classroom). Characteristic of oral professional legal communication is the bookish character of speech due to its qualification. This fact limits the number of functional styles included in the program and the syntactic and grammatical constructions that can be worked out.

In the process of organizing classroom lessons on teaching scientific, professionally oriented communication the control of linguistic, communicative and professional communicative competence was carried out separately. As the experiment has shown, this makes it possible to make the work on each thematic series more meaningful for the postgraduate and allows in practice to implement the principle of individualization of training and control. Thus, postgraduates with different initial language training are assessed on different scales of requirements only three scales; for "strong", "medium" and "weak". That is a master's student with a weaker language background can receive a grade of "five", which will testify the progress he made in relation to his own initial level of knowledge.

For postgraduates, applicants and adjuncts, there is a need not only to read scientific literature in English, but also to present their ideas and results in English themselves at international conferences, on pages of an international scientific journal, in an application for a grant or a joint project. It is known that translation is not always the best solution. The purpose of practical exercises is to fill this gap: create a scientific text on your own, study the stylistic features of scientific presentation, use a ready-made phrase, expression, phrase or model from a specially 
compiled list, cliché. The organization of a scientific text offers speech formulas, turns and expressions that are actively used in English when a research problem is formulated its material and methods are indicated, literature on the topic is discussed, experiments and data obtained in them are described, their systematization and explanation (interpretation) is made, conclusions are drawn. And also, in the learning process speech models of reasoning are given containing speech formulas, models and samples that show how scientific reasoning is built and what means give it coherence and completeness. All these is called "rhetorical", they highlight the main and the secondary, set the structure of the entire sentence, allow you to briefly, clearly and simply express the author's opinion, justify or explain the choice, highlight accents, derive consequences from it and much more. One of the training parameters is "metatext" comments, i.e. the ability to give annotations, summaries, reviews, comments, thesis, reports, etc.

Practical lessons in a foreign language at the postgraduate stage simulate the language competence of a native speaker, speech of the author of a scientific presentation, peculiarities of a scientific style in English and rules of idiomatic expression of meaning in the English language system. It also has a self-learning mechanism, that is, the principle of analogy, reproducibility, self-development, embedded into the language itself and ensuring its automatic, subconscious assimilation.

In general, the modeling of the process of formation of a foreign language communicative competence of lawyers in the system of postgraduate education determined the specifics of the corresponding methodology, the leitmotif of which is the idea of problem learning. It is to this idea that all the components of this technique are subordinated. The problem approach is based on a special organization of the actions of postgraduates to understand and resolve a problem situation, to find ways to solve problem problems of varying degrees of complexity, relying on previously acquired experience. This approach is possible as a reorientation of the teaching process from teaching to leaning.

In this regard, the fact was discovered that in scientific use the concept of "problem task" and "problem situation" are widely used, which are considered as fundamentally different since they denote different psychological realities. The first of them is characterized by the presence of a contradiction, the overcoming of which is associated with the mobilization of cognitive activity and mental processes, inclusion of elements of creative search, thinking and speech activity on the one hand, mastering new knowledge and methods of obtaining them on the other. The second assumes that there are several possible ways to resolve it. In accordance with this it can be assumed that the effectiveness of the formation of foreign language communicative competence of lawyers in the system of postgraduate education 
largely depends on the application of a problematic approach based on the interrelation of problem situations and tasks, as well as on a certain sequence of stages: the acquisition of knowledge (here, theoretical and methods); development of speech skills and abilities (communicative-activity method dominates); development of professional communication skills (preference is given to methods of problem learning).

The specificity of teaching languages in a sense limits the possibilities of problematic approach and forces the use of combined methods, that is, the adequacy of the application of one method or another is always determined by the focus of a particular stage of training on the development of language, speech or communication skills. Therefore, in our case, we consider it appropriate and most effective to use 1) method of professionally oriented teaching and 2) method of case study. This will be discussed in more detail in the second section. 


\section{CHAPTER 2. \\ EMPIRICAL RESEARCH ON METHODS OF FOREIGN LANGUAGE TEACHING}

\subsection{ANALYSIS OF METHODS OF TEACHING FOREIGN LANGUAGES FOR SPECIAL PURPOSES IN POSTGRADUATE EDUCATION PROGRAM}

Postgraduate professional education in the departmental regulations of the Ministry of Internal Affairs of the Republic of Kazakhstan means postgraduate and graduate education the purpose of which is to train highly qualified scientific and scientific-pedagogical personnel for the internal affairs bodies (State compulsory education standard of the Republic of Kazakhstan. Master's degree. Specialty 6N0303 - Law enforcement, 2004).

The rules for targeted training of doctors of philosophy $\mathrm{PhD}$ in institutions of higher and (or) postgraduate education were adopted in accordance with the Order of the Minister of Education and Science of the Republic of Kazakhstan dated May 22, 2012 No. 235 "On approval of the Mechanism for targeted training of masters and $\mathrm{PhD}$ doctors in basic higher educational institutions" changes and additions from 8.10.2018 (On Approval of the mechanism for targeted training of Master's and $\mathrm{PhD}$ Doctors in Basic Higher Educational Institutions, 2018).

The state compulsory standard of postgraduate education "Master" as amended by the Resolution of the Government of the Republic of Kazakhstan dated May 13, 2016 No. 292 - describes how the educational process goes, the scope of the curriculum disciplines, requirements for the level of training, the content of the educational program. The goal of postgraduate education is to prepare, taking into account the country's development prospects, competitive highly qualified personnel with high spiritual and moral qualities, capable of independent thinking and ensuring progressive scientific, technical, socio-economic and cultural development of society. Determined by professional competencies - knowledge, skills and abilities necessary for the effective implementation of professional activities in the law enforcement system and the relevant position (State compulsory standard of postgraduate education "Master" as amended by the Resolution of the Government of the Republic of Kazakhstan, 2016), (On the approval of state compulsory education standards of the corresponding levels of education, 2012).

A competent person can have certain skills or can acquire them by instruction or learning. There is, nevertheless, a difference between skill and competence, since the competence relates to behaviors observed (Stoof et al, 2002) and the competence with actions. Words like skill, capacity and action are used like synonymous of 
competence, whereas knowledge, skill and attitude are part of the competence. Westera (2001) defines competence as higher order cognitive skills and behaviors that represent the ability to cope with complex, unpredictable situations. Therefore, competence includes other processes such as metacognition and the strategic thought and presupposes an intention and conscious use of both. To reach competences implies being an expert apprentice and lifelong learning, to adapt to a world in continuous change (OCDE, 2010). The OCDE Program Definition and Selection of Competencies (PDSC) alludes to the achievement of a high degree of integration between the capacities and the extent of social aims that an individual has when pointing out that the competence is more than knowledge and skills. It also includes the skill of facing complex demands, relying on and mobilizing psychosocial resources (including skills and attitudes) in a particular context (Noguera, 2003).

Noguera (2004) and Fernandez - Salinero (2006) offer a definition of competence in linguistic terms, indicating the combination of attributes that concerns diverse orders of the person related with: a) the knowledge, aptitudes and technical skills (to know); b) the methodological ways of proceeding in an activity (to be able to do); c) the guidelines and individual and collective forms of behavior (to be able to be); d) the forms of organization and interaction (to be able to be).

In accordance with the State Compulsory Education Standard of the Republic of Kazakhstan, the goal of teaching foreign languages in postgraduate studies is determined as deepening and developing the acquired knowledge, skills and abilities of active command of a foreign language in varying situations related to the professional activities of students. Knowledge of the general scientific vocabulary necessary to express your point of view on the read scientific literature related to the specialty of a master's student, and to conduct a conversation on scientific and socio-political theme, on the theme of master's work (On approval of the Regulations on Postgraduate Studies, on Doctorate Studies, on the preparation of doctoral and candidate dissertations outside of doctoral studies and postgraduate studies at higher educational institutions of the Ministry of Internal Affairs of the Republic of Kazakhstan, 1998).

It is obvious that the National State standard is conceptually related to the principles set forth in the World Declaration on Higher Education for the 21st Century, adopted at the UNESCO World Conference in Paris in 1998 where institutions of higher education should provide students with an education that fosters well-informed and deeply motivated citizens who are capable of critical thinking, analyzing social issues, finding and using solutions to the problems facing society and to take on social responsibility. Education Ministers have also adopted the Paris Communiqué (2018) highlighting priority activities in this area 
for the coming years. The Communique outlines the joint vision of education ministers from 48 European countries for a more ambitious European Higher Education Area by 2020. It calls:

- for an inclusive and innovative approach to learning and teaching

- for integrated transnational cooperation in higher education, research and innovation

- for securing a sustainable future through higher education

In turn, in the development of the provisions of the State Educational Standard of the Republic of Kazakhstan, clause 1 of the Order of the Ministry of Internal Affairs of the Republic of Kazakhstan "On Approval of the Regulations on Postgraduate Studies, on Doctoral Studies, on the Preparation of Doctoral and Candidate Dissertations outside Doctoral Studies and Postgraduate Studies at Higher Educational Institutions of the Ministry of Internal Affairs of the Republic of Kazakhstan" determines the goal of postgraduate training of lawyers deepening theoretical and special training of scientific, pedagogical and scientific personnel, taking into account modern requirements for highly qualified personnel, mastering methods and means of scientific research, developing the ability to independently conduct scientific, pedagogical and educational work at a high level (On approval of the Regulations on Postgraduate Studies, on Doctorate Studies, on the preparation of doctoral and candidate dissertations outside of doctoral studies and postgraduate studies at higher educational institutions of the Ministry of Internal Affairs of the Republic of Kazakhstan, 1998). An adjunct during his studies in the post-graduate school of a university within the prescribed period must pass the candidate exams in a foreign language, the main requirement of which is the demonstration of practical knowledge of this language, which allows it to be used in scientific work.

Practical knowledge of a foreign language within the framework of this course requires the following skills:

freely read original literature in a foreign language in the relevant field of knowledge (jurisprudence - E.U.);

arrange information extracted from foreign sources in the form of translation;

make messages and reports in a foreign language on topics related to the scientific work of a graduate student (applicant) and conduct a conversation on the specialty and socio-political issues.

Studying the best teaching experience at the world leading universities (Imperial College Business School (Great Britain), Frankfurt School of Finance \& Management (Germany), HULT Business School (Great Britain), University of Applied Sciences Mittelhessen (Germany), Rotterdam School of Management (Holland) showed that in the listed universities any subject is presented in a 
refraction with reality, and is not a set of basic knowledge that you can read in a textbook but various trainings with real examples from practice (Abdrasulov \& Seytaeva, 2016).

It should be noted that the overwhelming majority of teachers understand the need for systematic mobile adaptation of foreign language teaching technologies to the requirements of a dynamically changing social reality. In particular, in the essay "Cyborg Pedagogy in Cyborg Culture" K. Luke insists that since all of us will be swept off the side of the road and into the thick of the stream on the information superhighway, take responsibility for changing curricula and educational process taking into account these conditions (Luke, 1999).

Thus, returning to the terminology adopted in our research, it should be stated that both in the intradepartmental normative acts of the Ministry of Internal Affairs of the Republic of Kazakhstan and in the normative acts of the Ministry of Education and Science of Kazakhstan regulating the activities of the postgraduate training system for lawyers, we are talking about the further formation and optimization of the competence of law enforcement officers in Kazakhstan, which is especially clearly manifested when comparing the requirements of foreign language teaching programs at the university and postgraduate stages (Table 3).

Table 3. The content of teaching a foreign language in Law school:

\begin{tabular}{|c|l|l|}
\hline & $\begin{array}{c}\text { Requirements for GRADUATE } \\
\text { education }\end{array}$ & \multicolumn{1}{|c|}{$\begin{array}{c}\text { POSTGRADUATE study } \\
\text { requirements }\end{array}$} \\
\hline 1 & \multicolumn{1}{|c|}{$\begin{array}{c}\text { 2 } \\
\text { Dialogue. A student can maintain a } \\
\text { conversation on a given theme for } \\
3-5 \text { minutes using adequate } \\
\text { expressions at a normal pace, show } \\
\text { interest, justify his opinion. }\end{array}$} & $\begin{array}{l}\text { Dialogue. The master's student is } \\
\text { able to take a multifunctional } \\
\text { participation in a non-time-limited } \\
\text { dialogue (polylogue) of everyday } \\
\text { life, general scientific and narrow } \\
\text { professional nature. Is able to solve } \\
\text { in the process of communication } \\
\text { one of the selected tasks from the } \\
\text { problem-communicative field of a } \\
\text { legal orientation. }\end{array}$ \\
\hline $\begin{array}{l}\text { Monologue. The student can tell the } \\
\text { story, the plot of the book he read, } \\
\text { the film and express his judgment, } \\
\text { opinion, attitude. Can make co-say } \\
\text { your judgment, opinion, attitude. } \\
\text { Can make a report on a project, }\end{array}$ & $\begin{array}{l}\text { Monologue. The postgraduate is } \\
\text { able to evaluate and comment on an } \\
\text { authentic specialized text of a legal } \\
\text { orientation, make a presentation on } \\
\text { the topic of scientific research. }\end{array}$ \\
\hline
\end{tabular}




\begin{tabular}{|c|c|c|}
\hline & $\begin{array}{l}\text { contract, concept. Provide } \\
\text { clarifications. }\end{array}$ & \\
\hline$\frac{b}{2}$ & $\begin{array}{l}\text { The student understands the main content } \\
\text { of oral messages on everyday topics, } \\
\text { topics of general professional nature, if } \\
\text { the speaker's speech is clear and distinct. } \\
\text { Can highlight the main content of TV and } \\
\text { radio programs on topical topics presented } \\
\text { by a professional speaker. }\end{array}$ & $\begin{array}{l}\text { The postgraduate understands monologue } \\
\text { and dialogical polythematic speech with } \\
\text { varying clarity and tempo. Able to work } \\
\text { with any authentic scientific audio text of } \\
\text { a legal orientation. }\end{array}$ \\
\hline$\sum_{\frac{1}{2}}^{2}$ & $\begin{array}{l}\text { Introductory, search, viewing, studying } \\
\text { reading fragments of texts in the specialty } \\
\text { (with detailed understanding using lexical } \\
\text { and grammatical analysis or translation). } \\
\text { The student can extract interesting and } \\
\text { given information from the read text and } \\
\text { use it in oral and written speech. }\end{array}$ & $\begin{array}{l}\text { The postgraduate knows all reading } \\
\text { techniques, is able to extract information } \\
\text { explicitly and implicitly expressed in an } \\
\text { authentic scientific text of a legal } \\
\text { orientation and use it both in the } \\
\text { conditions of educational oral and written } \\
\text { speech, and in a situation of real } \\
\text { discussion and original scientific } \\
\text { research. }\end{array}$ \\
\hline ש & $\begin{array}{l}\text { Writing a small letter of a personal nature, } \\
\text { containing 2-3 paragraphs, annotations, a } \\
\text { short summary, an abstract of the text } \\
\text { read. }\end{array}$ & $\begin{array}{l}\text { A master's student is able to record in } \\
\text { writing information of a legal orientation } \\
\text { of any complexity, annotate, summarize, } \\
\text { abstract an authentic specialized text, use } \\
\text { the information received in an } \\
\text { independent scientific research both in its } \\
\text { original form and in the form of } \\
\text { translation. }\end{array}$ \\
\hline
\end{tabular}

It should be noted that, despite the obvious urgency of the problems of postgraduate professional education and even the presence of appropriate programs this aspect of the didactic theory has not been given due attention to this aspect of the domestic pedagogy: the concept, system-forming principles and model of the formation of foreign language communicative competence have not been defined and developed a professional lawyer. The State Educational Standards of the Republic of Kazakhstan for the preparation of postgraduates in the specialty 6N0303 "Law Enforcement" (Arthur, Brennan \& de Weert, 2016) has identified two main specializations of training, such as: profile and scientific and pedagogical (Figure 13). 


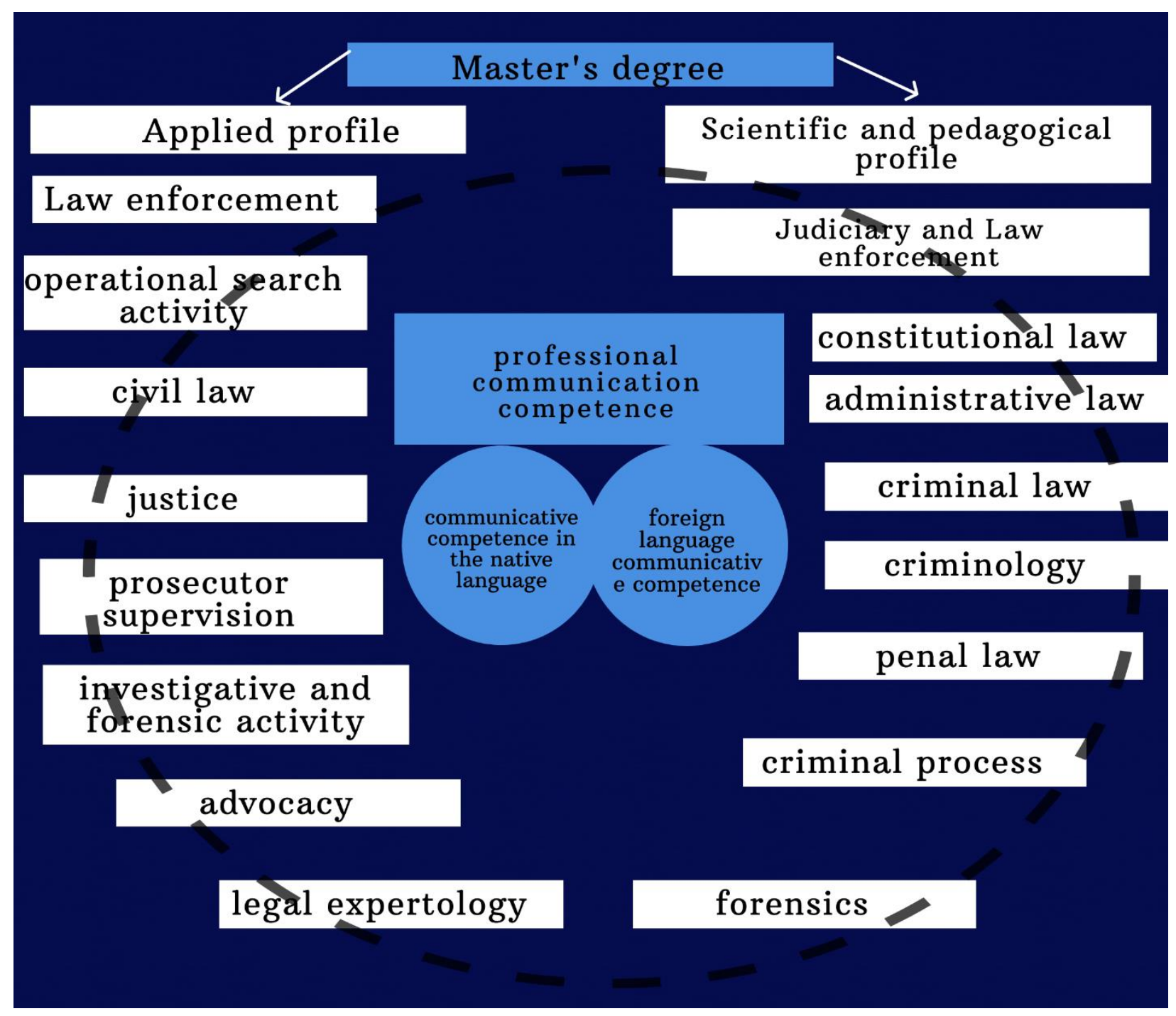

Figure 13. Model of a postgraduate program in Law

The content of the educational process of a master's degree program in Law and the foreign language communicative competence of the latter determined by State Educational Standards of the Republic of Kazakhstan is in complex dialectical relationships. On the one hand, the content of education is the subject component of professional communicative competence, on the other hand, the parallel formation and optimization of the communicative competence at the postgraduate stage is the most important. As it seems to us that, it is a condition for the creative development of the specified content and for the successful implementation of an original scientific research by a master's student (Uteubayeva, 2010). A similar picture, from our point of view, is observed in the field of professional spheres of lawyers (Figures 13 and 14). 


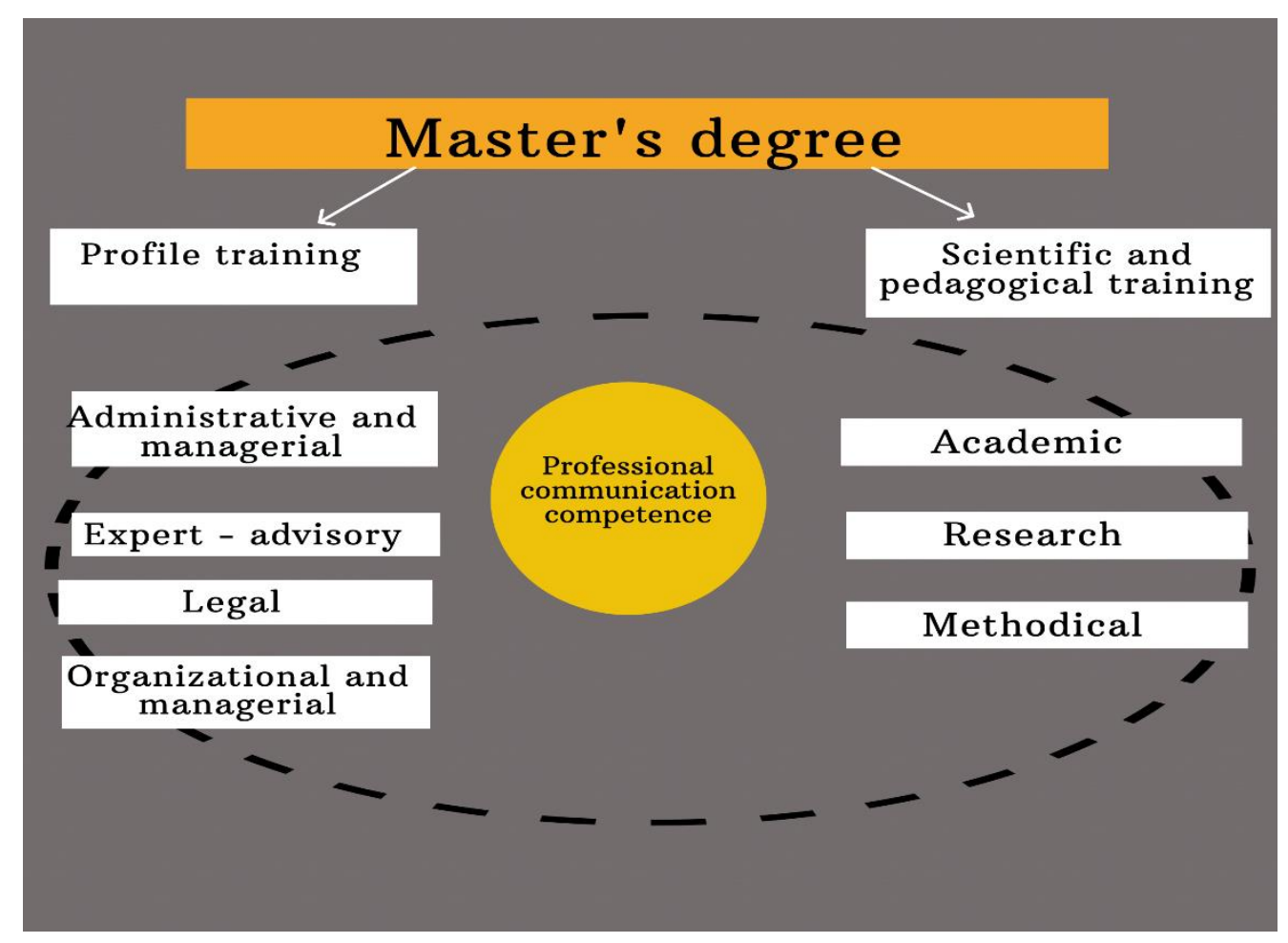

Figure 14. Types of professional activities of training lawyers

Mastering the general skills of interpersonal foreign language communication, without which successful scientific and professional activity is problematic, is certainly impossible without the practical mastering of the language and, in general, sign systems and conceptual apparatus.

Thus, the basis for optimizing foreign language communicative competence of lawyers at postgraduate stage is the mastery of linguistic competence in the process of university education carried out systematically and purposefully through special courses in the native language. All academic disciplines aimed at developing communicative competence among law enforcement officers, the term "literature" means that future lawyers should know how the language works; what patterns to use in oral and written form; and most importantly how to make the word an effective means of achieving non-speech goals in law enforcement and other legally significant situations.

To learn a conscious attitude to the word, to develop and consolidate the skills of fluency in oral and written speech in professional communication, we need:

- to characterize the word as an important component of the worldview of any educated person;

- to get acquainted with main provisions of general theory of a word and, with their help, explain the emergence and development of the textual form of law; 
- to present language as an innate ability of a person, a higher mental intellectual function, which is improved as new knowledge and experience are acquired in the processes of personality socialization;

- to show which properties of the word are most significant for the legal sphere of communication in general;

to formulate general and special rules of speech behavior of lawyers" (Gubayeva, 1995).

At the same time, theoretical knowledge is involved in the development of communication skills and abilities indirectly, as part of practical skills that serve as the basis for generalizing theoretical knowledge. Thus, theoretical knowledge about the language system, about certain grammatical categories contributes to the formation of linguistic intuition, which is a link of conscious or unconscious control over the correctness of speech activity, due to the presence of linguistic competence in postgraduates.

We rely on the already formed professional communicative competence teaching foreign languages at the postgraduate stage, the most important task of this period is to optimize the skills of postgraduate, using units of different language levels, to generate coherent texts adequate to the professional situation and within the limits of natural automatic error correction, admissible for a given language (General rhetoric, 1986).

In addition to the ability accurately form a coherent text, communicative competence also involves the speaker taking into account the sphere of communication, the situation and type of communication, the topic of speech, which together constitute the field of communication. Taking into account the goals of postgraduate stage of training lawyers (Leontyev, 1980), we believe it is especially important for the optimization of foreign language communicative competence to acquire the skills of judicial and scientific communication which have their own specific characteristics.

Scientific literacy is a major goal of science education at postgraduate level. In attempting to find an appropriate meaning of scientific literacy, several definitions have been proposed (DeBoer, 2000), (PISA, 2003). Science educators have relied on standards-based definitions of science literacy and have defined science literacy as scientific attitudes and the abilities needed to build an understanding of science in order to apply these ideas to realistic problems and questions related to science and law, as well as to inform and persuade other people to take action based on these scientific ideas. One important means of independent learning is the capability of successfully implementing high-order skills such as inquiry and problem-solving skills (Schneider et al., 2002) as well as thinking and learning skills (Campbell et al., 2000). Likewise, the Zohar and Dory (Zohar \& Dori, 
2003) emphasize that high-order skills and "high" literacy is essential to address the complexities of modern a life. Since information and knowledge grows much faster, than ever before, the meaning of the word "knowledge" has changed from the ability to remember and repeat information, find and use it effectively.

The scientific communication focuses on oral and written communication skills, e.g., the processes of speaking, listening, writing, and reading. These abilities are highly valued within the scientific community. Scientists who communicate well are successful in gaining support from members of their own communities, funding agencies, and society at large. These abilities are greatly needed also in scientists' daily lives. For example, the unique features of the free flow and the unedited World Wide Web increase the need for readers with sufficient knowledge, metacognitive awareness, critical thinking, executive control of their reading comprehension, and proficiency with diverse learning abilities (Yore et al., 2013). We tried to develop for postgraduates the level with the aim of acquiring learning skills by integrating them into the science studies. It supposed to be focused mainly on the following skills: information retrieval, scientific reading, scientific writing, listening and observing, information representation, and knowledge presentation. We refer to these skills as 'scientific communication skills'. Like Garvey and Griffith (1972), who argued that "Communication is the essence of science", we also refer to these basic skills that scientists need to master to communicate your findings and ideas. Since scientific literacy the main purpose of science education, an important component is teaching postgraduates to share their knowledge and ideas, as scientists do.

The characteristic features of scientific communication are determined by the fact that in a scientific discussion both the producer and the recipient of the speech are specialists working in a certain scientific field, and in the texts of scientific content they act as addressees, then addressees of speech with equivalent conceptual and logical thinking the nature of speech interaction, dominants of which should be recognized as generalized abstraction and emphasized consistency and due to the latter accuracy, objectivity, formality, standard scientific speech is not authoritarian unlike judicial communication the main task of which is in a broad sense, regulation of legal relations the main function of scientific communication is the message, fixation of results of knowledge of the world; in contrast to judicial communication, which contains an unconditional pragmatic program, reflecting the author's calculation of the perception of the text by the addressee, or, in a filmed form a certain model of the addressee, i.e. the connection "text - text recipient" (Matveeva, 1990) the dominant of scientific communication is a rational program that contains information about the subject of speech, i.e reflects the relationship "text denotation of the text". 
However, despite rather significant differences between the analyzed types of communication language proficiency presupposes at least three conditions:

1) ability to express meaning in many linguistic means;

2) ability to derive meaning from a given sentence, to unmistakably recognize synonymy and homonymy;

3) ability to distinguish true statements from false ones, correct sentences from incorrect ones.

Since the field of communication (the structure of judicial and scientific communication) is a set of interrelated elements that determine the choice of linguistic means and ensure the unity of processes of purposeful communication it is necessary to determine the complex of professional communication skills and abilities that must be actualized in the process of postgraduate education. To develop a foreign language course program for postgraduates and adjuncts of universities of the Ministry of Internal Affairs of the Republic of Kazakhstan a set of professional communication and speech skills and abilities of a law enforcement officer should be specified.

The professional activity of a lawyer (practice and researcher) is wide and varied: it includes both individual techniques (for example, specific methods of searching for information) and systems of techniques the possession of which is necessary in the process of implementing certain areas of a lawyer's work. Therefore, in the specialized literature, as a rule, the discussion of a complex of professional skills and abilities is directly related to a specific field of activity that is the selection and development of elements of a lawyer's working technique can be carried out depending on the real needs of professional work and the duties performed by a lawyer. This research is not intended to identify the full range of skills and abilities that the applicant should optimize. We will dwell only on those communicative skills and abilities that, in principle, can be correlated with the methodological concepts of linguistic and communicative competence. That is directly related to the knowledge of the system of the native and foreign languages and its adequate use in specific situations, so that they can be highlight the complex of requirements in the space of interdisciplinary training. The general concepts of communicative, in particular foreign language competence highlighted above (ability to speak, listen, read, write) should be filled with specific content that allows you to develop an adequate system of tasks.

Taking into account the above, the following main areas of work of a lawyer can be distinguished, directly related to the language:

- work with fixed information (including work with legislation and technology for studying documents, scientific publications);

- interpersonal communications of a lawyer (legal researcher). 
The effectiveness of legal (practical and scientific) activities is directly related to how the work with fixed (fixed in the relevant sources) information is organized. The object of work with fixed information turns out to be texts that are diverse in meaning and content: laws, decrees of the President of the Republic of Kazakhstan, resolutions and orders of the Government of the Republic of Kazakhstan, instructions, criminal cases, statements of claim, claims, objections to claims, contracts, various acts, statements, statistical data, scientific publications, etc. It should be borne in mind that the texts contain legal and non-legal, normative and non-normative, initial and processed, factual and evaluative information, i.e., firstly, texts can be written using a wide variety of functional styles, and secondly, fixed information is ambiguous, contradictory, in many cases incomplete and often needs to be transformed which requires extensive mental work.

The main stages of working with fixed information are:

- understanding of law;

- search for the necessary sources of information, i.e. publications and texts, unpublished or, in principle, unpublished documents and materials;

- selection of the necessary sources and texts of information mainly on the basis of relevance to the duties performed and the tasks to be solved;

- assessment of the state of sources and texts;

- analysis and processing of information in intellectual and technical terms;

- use of information.

Experts note that the stages of working with fixed information are filled with specific content also depending on the type of activity of the lawyer, the content of the information itself, the conditions for obtaining it, the nature of the problem being solved, etc. The goals and methods of work, the nature of the required qualifications, the amount of time required, the technical means used to work with information are changing. Thus, the investigator before referral to the prosecutor, the defense attorney when familiarizing himself with the case, the judge at the cassation instance, read the same criminal case differently and use different working methods (Minaeva, 2007).

We can single out some basic general rules and techniques for working with sources that do not depend on a specific professional field of activity without discussing specific professional rules for working with information. In accordance with the identified stages of work we will consider which techniques and techniques prevail at each stage.

The main problems of understanding law are solved by the theory of law and branch legal sciences. Understanding of the law is a prerequisite for working with legislation and must correspond to a level that meets the standards of professional training determined by the requirements of society for a lawyer. It represents work 
with the legal information itself, clarification of its internal meaning, i.e. the sum of meanings, content of prescriptions, establishment of possibilities for the implementation of the law, its managerial potential. This stage assumes not only a thorough knowledge of the main sources of law, but also the ability to analyze texts, compare newly published documents (when it comes to working with legislation) with the previous options.

The search for the necessary sources of information involves obtaining complete and consistent, relevant and systematized information. In the field of working with legislation, in particular, the following difficulties may arise:

- distinguishing between legal and law enforcement acts;

- recognition of the text as a source of law;

- search for text in an integral array of normative legal acts.

The above difficulties require a lawyer to be able to make an adequate selection of necessary sources and texts of information, assuming the applicant has:

- professional specific criteria for distinguishing sources;

- ability to clearly and correctly determine the tasks to be solved in each specific case.

Assessing the state of sources and texts, analyzing and processing information intellectually and technically require certain skills in working with written documents. Examining documents includes:

- identification of the author (collective or individual) of the document (source);

- assessment of its actual completeness, objectivity, truthfulness, consistency;

- understanding the meaning of the document (source);

- formulation of conclusions about the nature of the information contained in documents (sources) and the possibilities of its use.

All of the above can be translated into a set of working techniques for studying - reading documents. In special literature the following working methods are distinguished:

- determination of reading goals, selection of search orientation;

- isolation of semantic information blocks and here the identification of the actual circumstances and actual composition of the message is of paramount importance;

- identification of sources of information available in the dossier, their origin, legal value, meaning, etc.;

- fixation of the extracted information;

- writing a short summary / essay. 
Some of these techniques, in their specific embodiment are of a general nature and should be adapted to the work of a lawyer; others are inherently legal and technical. Nevertheless, rationality and quality of familiarization with documents (reading, studying a case, publishing) depend on professional preparedness of a lawyer (practitioner and researcher), his ability to read, differentiate and memorize legal information, highlight semantic connections, gaps, etc.

The following stages of reading work are distinguished:

- attribution (if it is unclear);

- determination of interests and positions of authors of the document (publication);

- understanding the reasons for the appearance of the document (publication) and its chains;

- Structuring the information received.

The result of studying the documents is the preparation of a short summary, which implies the mastery of the technique of describing documents and drawing up a summary of them. The final material can be put on in different forms using different styles. However most often it is carried out either in the style of a solution (project), or in the style of a conclusion (review, analysis, expertise, reference).

The highlighted methods of working with texts, mastering of which mainly takes place in the process of general training of a lawyer make it possible to determine methodological tasks in terms of methods and techniques of working with texts within the framework of a foreign language course at the postgraduate stage. In the present research, optimization of functional reading skills as a type of speech activity (in terms of the methods of teaching a non-native language) is determined by those requirements for the general techniques of a specialist's intellectual work which are presented in the general training program.

Communication in the work of a lawyer plays a primary role; almost all professional activities of a lawyer are implemented through interaction with other people, i.e. in the process of interpersonal communication. Suffice it to say that any text produced by a lawyer be it a draft agreement or a court verdict or a draft normative act must ultimately be announced brought to the attention, agreed upon, worked out jointly. Only in the process of interpersonal communication are negotiations between a lawyer and interested parties carried out, court cases are considered, opinions and decisions of lawyers are communicated to the addressees. We are not talking about those elements and qualities of interpersonal communication that are regulated by law (for example, procedural procedures for considering cases) and are embodied in status characteristics and procedural rules. Let us single out those cases of interpersonal communication of a lawyer that 
determine the effectiveness of solving the tasks undertaken (forming the court's opinion, convincing the client, reaching a compromise with a partner, etc.). Moreover, the professional training of lawyers is traditionally focused on the development of methods of various types of professional and non-professional interaction.

The methods of interpersonal communication common in the sphere of performance of official duties include: conversations, including explanations and interrogations; conversation; participation in peer discussion; speeches in colleges and collectives; receiving estimates, instructions, instructions from superiors or other subjects; joint implementation of any directions or types of work; informal contacts, consisting in mutual observation, informal assessments, informal assistance or opposition, work with participants in the legal turnover.

In this context, we can single out the general requirements for the interpersonal communications of a lawyer which should be based on some common language, the use of the widest possible set of formal and informal interaction tools, purposefulness, structural organization, information efficiency, consideration of possible interference from the participants in communication. In accordance with the general requirements listed above there are general interpersonal communication tools, namely:

$>$ language and in general used sign systems, conceptual apparatus, which should be, if not general, then at least perceived by all participants in the interaction; on the basis and within the framework of a common language, the choice of goals, awareness of interests and the formation of positions of the participants in the interaction are carried out;

$>$ strategy, tactics and demeanor of a lawyer in the process of interaction, including the ratio of persuasion, suggestion, selection and dosage of arguments;

determination of the system and limits of concessions in the process of interaction, acceptable results of interaction both for the lawyer and for his partners;

selection of suitable conditions for interaction, including place, time, presence of unauthorized persons, etc.

The specificity of using common communication tools depends on the goals of interaction, responsibility of a lawyer, degree of conflict of interaction, divergence of interests, personality traits of a lawyer, etc. The listing of some types of possible interaction shows not only the variety and levels of complexity of using communication tools, but also the variety of positions taken by a lawyer. The main types of interaction include interrogation of witnesses, suspects, accused; obtaining 
explanations from citizens, officials on various issues requiring the intervention of a lawyer; lawyer's explanation of his position on a particular issue, current law, and certain requirements for a particular subject of law is a participant in the legal turnover.

Professional oriented communication is a complex integral system that plays an important role in the life of a professional community.

The concept of communication in humanities is naturally associated with the concept of activity which has led to an active interest in these complex phenomena of researchers from various fields of knowledge about man. Fundamental studies of the theory of speech activity and functional stylistics Vinogradov V.V., Sirotinina O.B., L.V. Shcherba and others; culture of speech Golovin B.N., Ozhegova S.I. and others; teacher communicative behaviors Myers S.A., Martin M.M., Knapp J.L., A.A. Leontiev, G.G. Gorodilova and others; cognitive and affective learning Nasser F, Chory R.M., McCroskey J.C., Booth-Butterfield S., Payne S.K.; learning and academic performance Aylor B., Dobransky N.D., Frymier A.B., Tobell J., O'Donell V.L. and others provided an opportunity to improve methods of teaching languages, allowed to create methodological systems that are really capable of solving the problems of teaching communication skills and habits. In particular, Kazakh University of International Relations and Foreign Languages named after Abylai Khan developed a concept of foreign language education in the Republic of Kazakhstan (Kunanbayeva, 2005).

Speech communication distinguished at the beginning of the twentieth century by F. de Saussure as an independent component of interaction, became the object of close attention of researchers in the late 60s - early 70s of the XX century when structuralism as the dominant direction in linguistics (and in methodology) exhausted its possibilities. The works of J. Searl and J. Austin, creation of the theory of speech acts made it possible to radically change the focus of consideration of the statement. The statement traditionally viewed from the point of view of the relationship between content and plan of expression began to be considered from the point of view of extralinguistic reality. To simplify greatly we can say that from the point of view of a new approach central question in the study of the act of communication has become the question: what does X do when $\mathrm{Y}$ speaks? By what linguistic means does $\mathrm{X}$ achieve its pragmatic goals?

The Stanford Encyclopedia of Philosophy defines communication as the process of interconnection and interaction of common subjects, in which there is an exchange of activities, information and experience, abilities, skills and abilities, as well as the results of activities; one of the necessary and universal conditions for the formation and development of society and the individual (Cat, 2017). 
The specificity of verbal communication, its difference from other types of activity lies precisely in the fact that its object are other people, their consciousness, the system of motives, the emotional sphere, their attitudes and goals (Rubinshtein, 1946). As a kind of activity, all the features that distinguish any activity characterize verbal communication.

Accordingly, the main characteristics of communication as a type of speech activity is motive, goal, structure, means, object, product, result. The motivation underlying speech activity is closely related to needs. From the point of view of speech activity, needs are classified as follows:

a) needs focused on the object and purpose of interaction;

b) needs focused on the interests of another person or society as a whole;

c) needs focused on the interests of the communicator himself (Leontyev, 1974).

The need is objectified in the motive. Communication motives can also be divided into three groups. The first group includes the motives of communication, the purpose of which is to establish business ties with people for the implementation of the necessary joint activities. These are the motives of the so-called business communication.

The motives of the second group involve communication with another person or group of people to influence a person or a group of people. The motive of the third group is a person's need for communication which manifests itself in the desire to be understood, i.e. aimed at developing communication skills. For this research the most important was the identification of the goals of communication since the curriculum for the language course of the specialty was built in such a way that the postgraduates entered into communication with rather rigidly defined goals.

There are objective restrictions on the scale of methodological goals and ambitions in the process of teaching communication in the target language insufficient language training of postgraduates. Certainty (predestination) and limitedness of the content plan and possibilities of expression, controllability of the process by the teacher who is forced to constantly intervene in the communication process since he retains two plans: linguistic and communicative can lead to the emasculation of communication simple and primitive dialogical communication with control of lexical and grammatical correctness.

Communication activities in the process of teaching the language of the specialty are ideally focused on the development and improvement of professional communication skills. On the disclosure of intellectual and creative personal qualities and reserves of postgraduates in the process of discussing professionally significant information for them using forms of work organization that are 
meaningful to them the need and importance of mastering which for future professional activity they are aware.

Optimization of professional communicative competence at the postgraduate stage assumes that the applicant is able to determine his own position in the upcoming interaction and his own goals and objectives based on which he can extrapolate a possible script of action. In a reduced form this preparation can be described as finding out the following parameters:

- what will be sought from the lawyer;

- what the conversation will be about;

- what he himself wants to get from this conversation.

The very process of interpersonal interaction is also described in terms of technology, which includes:

- establishing contact based on an assessment of one's own position, the position of the interlocutor and meaning of the conversation;

- fixing the position of the addressee of the conversation (includes the inducement to transfer information, its differentiation, establishing a connection with the subject and goals of the conversation, its sufficiency and plausibility, reliability);

- formulation and justification of your own position (i.e., designation of the subject of the conversation, which is mandatory; indication of the competence and capabilities of a lawyer; putting forward proposals or requirements, the volume and nature of which may vary);

- opposition (questions, additions, elements of polemics, influence on the argumentation of the addressee of the conversation, that is, the addition of the interlocutor or objection to him);

- reaching a general conclusion.

In addition, as part of the general professional training of a lawyer it is necessary to pay attention and work out the general rules of conduct and communication. These include techniques that are honed through special preparation and targeted training, for example: showing self-confidence, reliability, benevolence, solidity; ability to instill in a partner a sense of greater security, freeing him from fear, feelings of hopelessness or uncertainty, an incentive to take the right action, etc.

The communicative skills highlighted in this subsection that are to be mastered in the process of training a lawyer, we called professional communication skills and abilities, and in the future it is this complex of skills and abilities that will be discussed as the basis for building a foreign language course program at the postgraduate stage. Thus, taking into account the general requirements for the formation of communicative competence in the native language of a law student it 
is possible to coordinate the tasks solved in the formation of a foreign language professional communicative competence of a lawyer at the postgraduate stage to specify the goals of teaching the language of the specialty to fill it with appropriate content and to determine the volume of communicative skills in accordance with what to choose adequate forms and types of educational activities.

Solving methodological problems special attention has to be paid to the formation of the types of speech activity defined in the space of interdisciplinary coordination and the field of research activities of the applicant which gives teachers the opportunity to choose ways to achieve their goals depending on the specific conditions of training and on the level of proficiency in the language being studied prior to the beginning of the course.

Motivation plays a special role in language teaching which is the motivation to take action to achieve personal or group goals. The problem of motivation is widely discussed not only in the methodology but also in all cases when it comes to managing the activities of other people. Motivation is a dynamic process undergoing constant changes and it is of utmost importance for the researchers to do their best to keep up with the dynamics. The social and educational context in which learners find themselves must also be taken into consideration. However, research work and gathering of research data is the best road to understand the phenomenon. In spite of many definitions of motivation there is still no single widely accepted definition of motivation. In 1972 Gardner and Lambert singled out two types of motivation: instrumental motivation and integrative motivation. They defined instrumental motivation as learning the language for more immediate or practical goals and integrative motivation as learning for personal growth and cultural enrichment. In 2006 Gardner defined motivation as a general characteristic of the individual that applies to any opportunity to learn the language. His socio-educational model conceptualizes motivation as the sum of the effort, desire to learn and positive attitudes. The development of the theory of motivation in the theory and practice of management ran into the following difficulty: a systematic study of motivation from a psychological point of view did not make it possible to determine exactly what prompts a person to work (Avanesov \& Karas,1996). However, studies of human behavior at work have made it possible to put forward some general explanations of motivation, which led to the creation of pragmatic models of employee motivation in the workplace. Meaningful theories of motivation are based on the identification of internal drives (needs) that make people act this way and not otherwise.

One of the most popular theories of motivation to this day remains the concept of Abraham Maslow, created in the 40s of the XX century, in which basic needs are reduced to five main categories:

- physiological needs the satisfaction of which ensures survival; 
- needs for security and confidence in the future;

- social needs, called the needs of belonging (include a sense of belonging to someone or something, a sense of social interaction, affection and support);

- need for respect (including need for self-esteem, personal achievement, competence, respect and recognition from others;

- needs of self-expression (need to realize their potential and growth as a person).

The last three categories of needs, according to A. Maslow, underlie many works devoted to the theoretical substantiation of the importance of taking into account motivation in language learning (Maslow, 2013). In the theory of P.Ya. Halperin, describing the main stages of the process of assimilating knowledge the first stage is also motivational (Galperin, 1988).

The success of training largely depends on how much the teacher is able to take into account emotional and motivational factors in his work (Leontyev,1972), Yakobson, 1969). For example, L.A. Yacobovitz (1976), discussing the problem "What does it mean to teach a language?" cites experimental data from research undertaken in the 60s to find out the share of various factors in the effectiveness of learning.

The results of the questionnaire can be discouraging for any methodologist because even with the most unjustifiably optimistic interpretation the role of methodology can be correlated only with those $14 \%$ that were included in the "Other". All the more important is the task of maximizing the use of motivational resources. The value of the results is determined by the fact that they are not tied to a specific educational institution, stage of training and applied approaches.

M. Fumadel determine the meaning of such a phenomenon as motivation, introduces the concept of valence, positive or negative. The individual, depending on his inclinations and aspirations receives a positive valence that corresponds to the goal set by him; on the contrary, negative valence is associated with phenomena that cause an instinctive or innate avoidance reaction in him (Shchepkina, 1976). Valence is determined by the individual's attitude to a particular event, that is, we can say that motivation determines the individual's behavior depending on which valence, positive or negative, certain phenomena and situations receive from him. Motivation leads to the desire to achieve personal results determined by the goal or motivating object, i.e. we can talk about the level of motivation (the limit to which the individual aspires to convince himself that he has achieved the desired result). M. Fumadel emphasizes that for an individual it is important to achieve success individually and not collectively (or from outside) the set goal. The initially set goal has a positive valence only if the individual is aware of his involvement and 
independence in achieving it, i.e. too easy or too difficult to achieve the goal acquires a negative valence.

M. Fumadel identifies several types of motivations that affect the effectiveness of teaching:

- cognitive motivations that meet the needs of perception;

- social motivations, manifested, first of all, in the desire for contacts with people and then, at the next stage, in the reaction to the environment;

- affective motivations, especially important between the ages of 10 and 18 , when an individual creates his own system of values and builds relationships in his sociolinguistic environment;

- negative motivations associated with the desire to eliminate or avoid the unpleasant (Vitt, 2002).

Considering that for the formation of motivations it is extremely important to know the goals that the student defines for himself, M. Fumadel (1976) suggests including the student in the process of developing control tasks. This conclusion is especially important today, when the coordination of the student's personal goals and the goals of the educational program becomes one of the primary educational tasks, the solution of which ensures the effectiveness of training.

The results of numerous sociological studies carried out in recent years and dedicated to clarifying the possibilities of increasing the effectiveness of teaching indicate that among the factors that improve the educational process, the following are the most important:

- introduction of new or correction of existing training courses and programs, taking into account the opinions of students;

- introduction of new teaching methods

The problem of motivation, i.e. systems of motives encouraging to learn a language is not new, we can say that the development of any technology for teaching a foreign language implicitly or explicitly includes the substantiation of the possible motivation of students. The problem of motivation is especially acute in nonlinguistic universities, where language acts as a non-core discipline.

Analyzing the problem of the role of motives and emotions in the success of language learning, Vitt (1973) singles out the main motive in the general structure of motivation, which may be the desire for knowledge, for obtaining a qualification, for the purposeful assimilation of knowledge and skills, for fulfilling the requirements for an applicant for an academic title. It is the main motive that prompts a person to act even contrary to his immediate desires, situationally developing emotions. The complex of private motives that form the whole system in the author's opinion are motives associated with the content of educational 
activity and its implementation, as well as motives determined by the relationship with the environment.

Motivation and cognition were treated as separate concepts throughout most of twentieth-century psychology. However, in recent years researchers have begun viewing the two as inextricably intertwined: not only does what we want affect how we think, but how we think affects what we want. In this innovative study, Beswick presents a new general theory of cognitive motivation, synthesizing decades of existing research in social, cognitive and personality psychology. New basic concepts are applied to a wide range of purposive behavior (Shchepkina, 2017)

N.I. Vitt (1973) proposes establishing direct links between educational activities characteristic of a particular university and educational work on mastering knowledge and skills in a foreign language class as ways to form high motivation in teaching a foreign language as a "non-core" discipline. A high level of motivation can be achieved by establishing links between the direction of scientific research of the applicant, related issues and topics and methods of conducting classes in a foreign language at the postgraduate stage.

The establishment of interdisciplinary connections seems to be decisive in the formation of a foreign language professional communicative competence at the postgraduate stage. By the term "interdisciplinary connections» we mean the didactic principle, specific structure of the content of training, a condition for increasing the effectiveness of training. It should be noted that interdisciplinary connections represent one of the specific forms of the general methodological principle of consistency, which determines a special type of mental activity systemic thinking. According to A.S. Belyaev (1997) interdisciplinary connections are not only a necessary condition for the development of a knowledge system but also the most important means of their implementation in the learning process contribute to the completeness and strength of knowledge and flexibility of their application. This is because interdisciplinary connections have the properties of universality their effect extends to all academic subjects and the study of each topic may include some form of connection with other disciplines. Different researchers carry out the systematization of interdisciplinary connections in different ways: based on the content of academic subjects and on aspects of general theory, laws and concepts, etc. Many science educators have perceived the necessity for a crucial paradigm shift towards interdisciplinary learning as shown in science standards. Interdisciplinary learning in science is characterized as a perspective that integrates two or more disciplines into coherent connections to enable students to make relevant connections and generate meaningful associations. There is no question that the complexity of the natural system and its corresponding scientific problems necessitate interdisciplinary understanding informed by multiple disciplinary 
backgrounds. The best way to learn and perceive natural phenomena of the real world in science should be based on an effective interdisciplinary teaching (Hye Sun You, 2017).

The types of connections are determined by the methods of mental activity and accordingly the establishment and substantiation of interdisciplinary connections based on the desire of postgraduates for cognitive activity is put forward as the main motive.

An almost simple and effective method is to establish a connection between the content of the educational material (texts) in the target language and the scientific issues underlying the research of the applicant. Taking into account the real conditions of teaching at the postgraduate stage (limited number of hours, lack of a language environment, different levels of language proficiency in one group, lack of time for the applicant, etc.) it is necessary that the student is aware that mastering a large number of units the sublanguage does not ensure at all mastery of a non-native language. On the one hand this may lead to a rejection of the desire to know really the target language; however, on the other hand, the motivation for learning the "sublanguage" will be high. In addition, one of the ways to form high motivation in language learning can be the intensification of learning meaning of which is to establish links between topics of foreign language classes and problems considered by the student. Undoubtedly, such an increase in the effectiveness of the process of forming professional foreign language communicative competence at the postgraduate stage is possible only on the basis of special abilities developed at the university stage of education the formation of skills and abilities defined in the educational standard $[69,1988 \mathrm{~b}]$.This includes the flexibility of thinking and the professional communicative competence of students implying the formation of complex intellectual skills in processing source material both orally and in writing.

An increase in the student's motivation at the postgraduate stage becomes real only if the tasks performed by the student are certainly necessary for the overall success of the further scientific activity of the subject of study. The applicant should be completely clear how he can achieve the goal, what he will learn and how useful the acquired knowledge and skills are [97, 2016b]. This approach does not increase motivation, language learning does not fall out of the general system of training a master's student and an adjunct who must be able to extract the main idea from the text material, briefly formulate the main content, be able to navigate in various texts [97, 2016c]. Among these skills for a lawyer engaged in scientific activity, such as compression, deployment, directed change of content, completion, addition, joining his opinion to the presentation of the text are especially important. During training in various types of "processing" of text, the applicant's speech is further developed since the latter optimizes the skill of clearly formulating thoughts, expands the 
thesaurus, complicates the generated speech structures and increases the level of functional literacy.

S. Epifanova (2000) proposes to differentiate the motives of the educational activity of students into two large groups:

1) immediate - motives directly included into the very process of activity and corresponds to its socially set goals and values. They include cognitive motives and motives of personality development, expanding the possibilities of its selfrealization and self-improvement;

2) mediated - motives associated with goals and values that lie outside the activity itself, but at least partially satisfied in it. This group included social motives, motives of achievement, success and incentive motives.

S. Epifanova (2000) noted that the most significant for the successful formation of professional foreign language communicative competence is the awareness of the need for knowledge to achieve success in life and material wellbeing, as well as the desire to expand horizons and erudition and raise a cultural level. Motivations are closely related and the most significant values such as success, freedom, money, self-improvement, interesting work are the most significant in the process of mastering knowledge.

Assimilation is a critical issue for knowledge management. Knowledge can be collected, created, or transformed, but if it is not absorbed, the organization will not be able to act on that knowledge or realize its full potential value (O'Leary, 2003). Modern research on this issue suggests using the principle of problem learning as one of the main tools that contribute to the emergence of intrinsic motivation.

The research is based on taking into account the cognitive motives associated with the very process of educational activities in classroom lessons; motives associated with personal interests and needs, and motives generated by the social order of society. It is assumed that the program for teaching the language of the specialty drawn up on the basis of clear links with the problems of scientific research and in accordance with the general requirements for the level of the formed communicative competence increases the general level of motivation.

In addition, the differentiation of postgraduates by the level of proficiency in the basic language "at the entrance" and the types of work in the classroom that are adequate to the level of preparedness. It allows taking into account the factor of achieving personal goals since the applicant is assessed by the real results of his work depending on the original knowledge.

An equally important factor that determines the success of the formation of professional foreign language communicative competence of a student at the postgraduate stage is the definition of the teaching methodology. The concept 
"method" traditional in domestic pedagogy means a way to achieve a goal, an activity ordered in a certain way aimed at achieving a goal. Teaching methods are at the heart of the course curriculum, they determine the choice of types of work. The problem of teaching methods has been studied in the methodological literature for many decades, numerous studies are devoted to it.

Teaching methods are defined as a way of interrelated activity of a teacher and students, aimed at solving learning problems, as a generalized representation of the scheme of interaction between a teacher and a student, as a model of activity. According to I.L. Bim the method is the main structural-functional component of the teacher's and students' activities, the way of achieving a certain goal in teaching and learning (Bim, 1977). The teaching method is considered in the form of two subsystems: method as a direction in teaching and method as a combination of teaching and learning. In this study the concept of "teaching method" is understood in the meaning of a combination a synthesis of teaching and learning which meets modern requirements for teaching methods in higher education and at the postgraduate stage and allows you to most fully realize the goals and objectives of the experimental course. Methodological systems differ from each other not in the description of technological actions but in the orientation, principles of system formation (in our understanding, hermeneutic).

As traditional learning styles evolve with the emergence of differentiated learning, more and more teachers are adjusting their approach based on the learning needs of their students. These learning styles highlight the five main strategies that teachers use in the classroom, as well as the advantages and potential disadvantages of each. As Eric Gill proposes a few fundamental teaching styles most educators tend to use (Gill, 2020).

1. The Authority or lecture style. This model is teacher centered.

2. The Demonstrator or coach style. The teacher shows students what they need to know.

3. The Facilitator or activity style. This style trains students to ask questions and helps develop skills to find answers and solutions through exploration; it is ideal for teaching science and similar subjects.

4. The Delegator or group style. Guided discovery and inquirybased learning place the teacher in an observer role that inspires students by working in tandem toward common goals.

5. The Hybrid or blended style. This style follows an integrated approach to teaching that blends the teacher's personality and interests with students' needs and curriculum-appropriate methods.

When developing a program for the language of a specialty motivational factors are considered one of which is the choice of adequate teaching methods 
which, in turn, depends on the specific learning conditions, goals and objectives. It has been repeatedly noted in the methodological literature that the principles, methods and recommendations that are adequate to the conditions of a specific experiment or specific training conditions cannot always give the expected result when even individual components of training change (for example, organization of space, number of training exercises, etc.). However, learning conditions in the broad sense of the word are primarily the goals and objectives of this language course. Therefore, it becomes an important task to distinguish courses, for example, B.A. Lapidus (1989) distinguishes the following three varieties:

I) courses with a purely utilitarian or highly specialized purpose in the field of speaking;

II) courses that pursue the goal of ensuring the formation of normatively and functionally correct skills, the formation of flexible skills and abilities that allow solving on their basis a wide range of tasks of verbal communication, that is, not tied to specific situations or strictly limited communicative intentions, and also pursue the goal of laying the basis for unlimited subsequent self-improvement in the language;

III) courses in which the general educational problem is put forward (school courses).

One can agree with the author that the distinction outlined above is of fundamental importance, because fundamental conclusions for the teaching methodology must be drawn from it. If we compare the conditions described under No. 2 (mainly a language university) and the conditions under No. 1. Then it is easy to see that many of the recommendations justified in conditions No. 1 minimum demands on the quality of pronunciation, tolerance for so-called noncommunicative errors and the appropriate technique for correcting mistakes, excessive concern for ensuring the memorization of common phrases, etc., are completely unacceptable under conditions No. 2".

In the proposed classification there is no such variety as a language course of a specialty at the postgraduate stage the purpose of which is to form a foreign language communicative competence of a lawyer at the postgraduate stage.

When choosing methods, we focus on the communicative method in teaching. The development of this method abroad began with the 1969 Congress "Psychology of Teaching a Foreign Language" held in Cambridge. The basic requirements for the organization of the training process were identified as follows:

1. The central role should be assigned to the personality of the student himself (it is assumed that training begins with the needs of the student, principle presupposes a change in the relationship between a student and teacher which 
should be equal partners, the need for individualization of training is recognized) (Kitaigorodskaya, 1986);

2. The communicative orientation of training involves teaching the solution of not only language and speech problems but also the mastery of certain sociolinguistic and behavioral skills through the inclusion of role-playing games, discussions, that is through the use of language for practical purposes to solve nonlinguistic problems (Palmer, 1970).

At present methodology of FLT widely uses the communicative-activity method the foundations of which were laid by the psychologists S.L. Rubinstein, A.N. Leontyev, I.A. Winter (2009). This method is based on the following provisions:

1) student is at the center of learning as a subject of educational activity which implies taking into account the interests, needs and motives of students when determining the purpose of classes and methods of organizing training;

2) activity-based nature of training: organization of the educational process is aimed at setting and solving a specific educational problem (the object of training is speech activity: listening, speaking, reading and writing);

3) language classes are focused on teaching communication using language for the purpose of exchanging thoughts. Particular attention is paid to maintaining the need for the assimilation of professionally significant and of general cultural value information;

4) organization of the educational process meets the following requirements: exercises are used that maximally recreate professional situations that are significant for students; in parallel grammatical form and its functions in speech are mastered; teacher's behavior in the class is communicative.

The development of the communicative-activity method is so complete, theoretically beautiful and exhaustively deep that it would seem one can neglect the well-known warning of D. Obrecht (1976) who said that an applied scientist must show maximum ingenuity, determining what not to support, which theoretical doctrines not to take into account at all and which ones to approach with caution. Communicative language teaching was developed by teachers and applied linguists as a response to the shortcomings of audio-language and grammatical translation techniques. One of the hallmarks of communicative language teaching is the emphasis on communicative activities that contribute to language learning. These actions use real life situations to induce communication. Communication activity pursues real goals, such as finding and sharing information, overcoming barriers, talking about oneself and studying theme (Rhalmi, 2010). 
The history of the application of the communicative-activity method shows that the implementation in practice of the main provisions and recommendations is not always effective enough. As an example, one can cite the work in which the author quite convincingly analyzes the existing methodological tradition and opposes "methodical squats" conditional communication exercises and all existing methods combined believing that a language can and should be studied in a real information process, knowledge of the language should be secondary the result of objective activity.

Such examples are not isolated and are presented not in order to doubt the value of the theory of the communicative-activity method but in order to once again emphasize the importance of an adequate translation of theory into practice (especially since the question of the advantages of a particular method as shown above can only be discussed in connection with specific learning conditions).

Since the problem investigation concerns teaching the language of a specialty at the postgraduate stage, when developing the course, it was necessary to consider the specific requirements for teaching professionally - oriented communication in the target language. These requirements and recommendations should take into account the current state of methodological developments. Modern requirements for specialty language courses are based on the communicative-activity method and consider the professional orientation of the university.

Language courses for specialty usually include two stages:

1) main - a basic course of a non-native language;

2) introduction to the specialty (preparation for listening, recording a lecture in a specialty in a foreign language, or the formation of certain intellectual skills).

Teaching a language, taking into account a professional orientation, is based on the following provisions: interest in learning a language which concentrates the attention of listeners and ensures the strength of memorization; understanding the ultimate goal of learning and the desire to achieve it; attention and associative connections; positive emotions in the teaching process; multiple repetition, providing the formation of speech skills and abilities; different types of memory; reliance on mental activity, the cognitive need of listeners, which is a source of motivation for learning a language. In the educational process techniques are widely used that contribute to the intensification of learning: problem and communicative situations, conditional and role-playing games that stimulate the unprepared speech of students; elements of suggestology and relaxopedia (Khmelevskaya \& Yarnatovskaya, 1990).

The use of problem situations in the teaching method has recently taken shape in a separate direction called the languages of the problem-based learning (PBL). The concept of an approach is broader than the concept of a method. In the 
methodological literature it is noted that it represents a certain system associated with the methods and means in which everything concerning the mass media is used.

One of the constructivist approaches which used well against lecture-based education is problem-based learning (PBL) (Hamdan et al., 2014). PBL is an innovative, student-centered, self-directed approach to learning in which learners are asked to solve real-life problems in order to encourage learning (Shin \& Azman, 2012). Among the main characteristics of PBL are learners taking an active role in learning diminished role of teachers to facilitators (tutors), involvement of cognitive and metacognitive skills scaffolding through peer feedback and various team dynamics in PBL groups and later well-structured and defined PBL processes.

The main problem with PBL in language classes, as stated by Larsson (Larsson, 2001), was the simultaneous use of language as learning target and learning tool. Simply put the answer to the question of whether language can be used to learn language was uncertain. Another problem with implementing PBL in language classes was its open structure. PBL like other inquiry-based approaches to learning does not assume one correct answer to learning problems. If an answer can be used to solve the learning problem, it should be accepted by the tutor. Educators have used main characteristics of PBL and designed their own PBL tutorship models. Some of them it was found have misunderstood the concept of PBL and misapplied it. The main problems with these studies are improper problempresentation not following rules of higher order thinking, excessive aid by tutors and ignoring delicacies of learning language through PBL (Loghman Ansarian \& Fateme Shir Mohammadi, 2018).

The peculiarities of using the problematic approach will be considered in the second chapter in connection with the description of those forms of work that formed the basis for the creation of a special course on the development and improvement of professional foreign language communication skills and abilities (Uteubayeva, Zhetpisbayeva \& Pastushenko, 2011).

It should be added that when discussing the problem of teaching the language of a specialty we are talking about a non-linguistic university, that is, about a special group of students in the methodology (about non-philologists) for which the language being studied is not a subject of professional activity and can only conditionally be considered a means of communication (means, application which may be limited to training sessions). This circumstance, on the other hand, can be effectively used in drawing up a program of a special course in the language of a specialty since in principle language is inherent like no other academic discipline the phenomenon of "meaninglessness": it is studied as a means of communication, filling content, forms of work, etc. carried out through the use of the knowledge and forms of work that are recognized as adequate for a particular course. 
So, the study and analysis of the educational documentation of the university, the lingua didactic aspects of teaching a foreign language in the system of postgraduate education allow us to state the fact that there are objective opportunities for the educational process of higher education in the formation of foreign language communicative competence of lawyers. These possibilities can be maximally realized under the condition of purposeful development and systemic application of the methodology for the formation of the required competence. 


\subsection{METHODS OF TEACHING ESP: RECOMMEN- DATIONS AND DISCUSSIONS}

Modeling the process of forming a foreign language communicative competence of future lawyers in the system of postgraduate education determined the specifics of the corresponding methodology the leitmotif of which is the idea of problem-based learning. It is to this idea that all the components of this technique are subordinated. The PBL is based on a special organization of the actions of postgraduates to understand and resolve a problem situation to find ways to solve problems of varying degrees of complexity relying on previously acquired experience.

Research shows that higher education develops cognitive skills as the ability to solve complex problems, reason in open-ended questions and in critical thinking environments. According to some studies $70-80 \%$ of the critical thinking abilities of young people under 30 are acquired only in higher education and $80 \%$ of general competencies are acquired in secondary school.

From this point of view there is no mismatch between the goals of employment and the ideals of university education contrary to what some experts argue that the university should be extremely professional and respond to the immediate needs of enterprises. Or, conversely, some believe that the university is an ivory tower.

There is a Humboldt expression: "knowledge forms". A truly university education must henceforth be based on science since the basic qualities expected from a high-level specialist are the same as the basic qualities of a researcher. As a consequence, the general culture is paradoxically very "utilitarian" in the area of training. The science should be placed in the very center of professional training, giving everyone scientific brightness, without which the chosen profession for each of them will remain gloomy and boring.

If we want to develop our competencies this means a deep revision of our teaching methods. Methods other than mainstream courses should be used. In this sense the modern movement towards "learning through problems" seems desirable. This approach places the mobilization of knowledge at the center of the educational process, starting from the first year.

PBL is a pedagogical approach that has been widely applied in multiple educational contexts to support students' problem-solving skills (Yew \& Goh, 2016). In Kazakhstan PBL has been studied extensively across various disciplines including law. Moreover, based on our review of the literature the findings are also contradictory regarding the effectiveness of PBL in CE education in Kazakhstan. These findings lead to a critical question: What are the current instructional 
practices in CE classrooms? However documented observations of teachers' pedagogical behaviors and evaluation of their implementation of PBL are limited. This gap in the domestic education field should be addressed with much-needed empirical studies that evaluate the instructional practice and effectiveness of PBL application in classrooms. Therefore, in this research we introduced a multidimensional and multi-categorical classroom observation protocol. Using the protocol, we then described and compared how a teacher allocated her instructional time and the instructional behaviors she utilized in two different approaches: Traditional and PBL. We examined the use of languages, strategies, communication mode, language content, and type of instruction.

One of the main benefits of PBL for postgraduates is the development of critical thinking skills something that has been studied by researchers. For example, four studies incorporated the California Critical Thinking Disposition Inventory (CCTDI) (Facione, 1992) and its revised version (Chen, 2013) to measure how PBL impacts college students' critical thinking dispositions and skills in learning English. Ding (Ding, 2016) found that PBL significantly improved college students' critical thinking dispositions including truth-seeking, open-mindedness, analyticity, systematicity, critical thinking, self-confidence, inquisitiveness, and cognitive maturity. The three other studies (Zhang, 2018) provided evidence that PBL supported students' development of critical thinking dispositions including openness, analysis, and fairness, and critical thinking skills, such as analyzing and explaining. We found that PBL also benefited postgraduates' critical thinking dispositions of truth-seeking, self-confidence, and maturity, and critical thinking skills of interpreting and evaluating.

In this regard the fact was discovered that in scientific use the concepts of "problem task" and "problem situation" are widely used which are considered as fundamentally different because they denote different psychological realities. The first of them is characterized by the presence of a contradiction the overcoming of which is associated with the mobilization of cognitive activity and mental processes, the inclusion of elements of creative-search, thinking-speech activity on the one hand, mastering new knowledge and methods of obtaining them on the other. The second assumes that there are several possible ways to resolve it. In accordance with this it can be assumed that the effectiveness of the formation of foreign language communicative competence of lawyers in the system of postgraduate education largely depends on the use of the problem-based learning based on the relationship of problem situations and tasks as well as on a certain sequence of stages: acquisition of knowledge; development of speech skills and abilities; development of professional communication skills. 
The methodological basis for constructing classes of different types, providing problem-based learning are the standard requirements for the organization of classroom lessons:

- determination of the goals and objectives of classes (goals for the development and improvement of linguistic, communicative and professional communicative competence are prescribed separately);

- determination of the goals of actions performed to solve problematic tasks and situations;

- choice of a method of action to implement problem situations (choice of an algorithm of work and type of work, depending on the stage of work on the thematic series);

- evaluation of the result of actions in accordance with fixed criteria for each type of work.

The structural components of classroom studies for each of the types of work within the framework of problem learning are in principle described above in the form of work algorithms. As a rule, 10-30 minutes is enough to solve a problematic problem (depending on the level of problematicity). When determining the time allocated in the structure of the lesson for solving a problem it is necessary to take into account its complexity and the level of language training of postgraduates.

The problem-based learning is used to teach a foreign language to postgraduates using theoretical data that have been developed in the domestic and world teaching methodology for many years (Salkhanova, 2004), (AbulkhanovaSlavskaya, 1980). The problem approach is based on a special organization of the actions of postgraduates to understand and resolve the problem situation.

The advantages of the problem-based learning are so obvious today that the introduction of this effective form of organization and conduct of the educational process which allows each student along with high theoretical training and practical skills necessary to be able to identify and resolve problems within the scope of professional activity is considered by the departmental legislator as one of the main directions of reforming departmental education (Minaeva, 1991). These advantages have been experimentally substantiated in numerous studies that have proven that the problem-based learning in teaching contributes to the development of intellectual, speech-thinking and creative independence of students. Z.M. Tsvetkova (1971) notes that in order to ensure the proper development of thinking in a language university it is necessary to make the process of teaching a language more independent and more problematic. Obviously, the specificity of the language as an academic subject requires a corresponding adaptation of this general didactic method the essence of which is to find ways to solve problems of varying degrees of complexity based on previously acquired experience. 
In the methodological literature the concepts of "problem task" and "problem situation" are considered as fundamentally different, because they denote different psychological realities. A.N. Leontyev (1959) characterizes the task as a goal given under certain conditions while the problem in didactics is presented as a question that has arisen or posed to the subject the answer to which is unknown and is subject to creative search. The problem task contains, as a rule, a contradiction the overcoming of which is associated for the student with the difficulties of solving it the mobilization of cognitive activity and mental processes, the inclusion of elements of creative search, thinking and speech activity on the one hand, mastering new knowledge and methods of obtaining them on the other.

Problematic tasks can also be applied at the postgraduate stage, i.e. when postgraduates have the basics of theoretical and practical knowledge in a foreign language at the bachelor's level which can be used to develop thinking and communication skills with setting problematic tasks for them.

A problem situation presupposes the presence of several possible ways to resolve it as well as the active involvement of the student in the process of finding an answer to the question posed in the problem. A problem-based learning is possible as a reorientation of the learning process from teaching to teaching. Therefore, A.E. Melnik (1989) characterizes the specifics of the problem situation that the unknown is the central element of the problem situation. In order to create a problematic situation, the student must be faced with the need to complete a task (practical or theoretical) in which the knowledge to be assimilated will take the place of the unknown. The unknown is always characterized by some measure of generalization. A problem situation is characterized by the degree of difficulty of the unknown to be assimilated. The main indicator of the degree of difficulty is the degree of generalization of the unknown unknown the degree of generalization that a student must achieve in the process of discovering new knowledge in a problem situation. The indicator of generalization thus becomes an indicator of a problem situation an indicator characterizing the student's capabilities (creative abilities and the level of knowledge, skills and abilities he has achieved).

The formulation of problematic tasks does not yet ensure and does not lead to the emergence of problem situations. Psychologists emphasize that a problem situation is always included in the conditions of theoretical or practical activity and arises only in the process of solving a problem by the postgraduates themselves.

Modern training programs include as a rule, sets of problem situations, i.e. specific situations. The practice of their application in teaching became widespread and stood out in a separate approach the so-called "method of specific situations". The essence of this method lies in the fact that in contrast to the task which involves the search for a solution based on the described initial conditions and in accordance 
with a certain methodology for finding the correct answer in a specific situation, questions can be posed and solutions are given. However, the most important thing for her is that everyone is looking for his own answer to the questions posed and each decision presented in the situation may have a different assessment and be interpreted differently by those discussing the situation. The specific situation is not an example of the correct or wrong decision or behavior. It is a specific example for discussion. Therefore, a particular situation does not live on its own without a group discussion process. Even if the text of a specific situation or additional information hidden from students but known to the teacher contains an answer showing how life solved the problem, the answer to a specific situation in the educational process will be the discussion that took place in the process, and the conclusions that according to the results of the discussion will be done for themselves by the students (Vihansky, 2001).

Obviously when teaching communication skills (reading and speaking) the teacher's goal is to organize the speech activity of students in such a way as to create conditions for the performance of the posed speech problem task. This methodological task according to A.A. Leontyev (1999) is necessary to assume the subordination of the teaching methodology to foreign language speech communication to a system of interrelated problems acting as a chain of tasks of gradually increasing complexity. These tasks should permeate all aspects of learning and all methodological techniques used in it should be aimed at stimulating creative activity and minimizing mechanical, meaningless operations.

The methodologist strives for the use of problem situations in teaching communication skills not only activates the intellectual abilities of postgraduates in the broadest sense of the word but also makes it possible to organize classroom lessons as a process of developing communicative skills carries out with the help of cognitive motives of the postgraduates and in accordance with requirements for modern training programs taking into account the focus of scientific research of trainees.

The specificity of language teaching limits the application of the problematic approach forcing the methodologist to use combined methods, i.e. the adequacy of the application of a particular method is always determined by the focus of a particular stage of training on the development of linguistic, speech or communication skills.

Synthesizing the existing classifications of teaching methods, we can say that the course program uses two groups of methods:

deliberately practical methods;

combined methods. 
In the process of mastering foreign language communication thinking plays a leading role therefore, researchers note the need to take into account the principle of consciousness and communication, i.e. the need to organize training in such a way as to create conditions for learners' awareness of linguistic factors creating adequate ways of using the studied material in communication situations.

Thus, the experimental course is built in a certain sequence of using the methods: acquiring knowledge - theoretical and cognitive method; development of speech skills and abilities - communication and activity method; the development of professional communication skills is a problematic method.

During the experiment, a large place is given to the activity of postgraduates in the process of mastering lexical and grammatical material because conscious assimilation of linguistic phenomena is achieved provided that the learners actively and not passively master the educational (linguistic, speech, theoretical) material and operate on it in an independent professionally oriented speech. Orientation towards solving problematic tasks and problem situations is inextricably linked with the development of the cognitive abilities of students with the activation of their speech-and-thinking activity therefore, all methods and techniques of teaching are focused on stimulating mental and creative activity which involves the use of a system of tasks sequentially arranged according to the degree of complexity. Solving problematic tasks and situations requires the activation of knowledge and skills previously formed in postgraduates and the development of new ones. The issue of continuity in the formation of lexical and grammatical skills is solved using combined (at the stage of preparation for solving problem problems and situations) and problem methods. In addition, the use of special types of work within the framework of problem-based learning allows solving the problem of optimizing professional skills and abilities which is an integral part of the development of communicative competence in the target language (Slesareva, 1980).

Obviously work with problem situations is aimed at developing professional communication skills, i.e. is the final stage in the algorithm for working on a thematic series. Therefore, the degree of complexity and the set of possible solutions to the problems posed depend on how effectively the work on the development of language and speech competence was organized. In the methodological literature the question of which types of assignments can be considered "already problematic" and which only "preparatory" is resolved ambiguously. The same diversity of opinions is noted in the proposed classifications of problem tasks and problem situations. In this regard, the proposed by A.P. Babenko (1968) is a list of requirements that tasks included in a series of problem situations meet:

1) tasks that determine the motivational nature of students' speech;

2) tasks that determine the purposefulness of students' speech; 
3) tasks that orient students towards emotional and personal speech;

4) tasks orienting the speaker towards the listener

It is possible to take the proposed list as a basis despite the fact that the formulation of the requirements listed above allows their broadest interpretation and content. The advantages of this list are that it allows you to distribute and typologies problematic tasks and situations in accordance with the stages of work on a thematic series ensuring the controllability of the educational process, giving it a cognitive and search character, creating conditions for the active involvement of students in work at each lesson. The algorithm for working on a thematic series involves a gradual increase in the complexity of problematic tasks the complication of communicative tasks of vocational guidance.

Each thematic series includes a set of problematic tasks, a formal game and a set of specific situations which makes it possible to activate both the professional knowledge of the trainees and develop professional communication skills. It means the ability to evaluate one's own position the position of the interlocutor and the purpose of communication; the ability to fix the position of the addressee of the conversation; ability to formulate and substantiate their own position; ability to oppose (ask questions, supplement, influence the argumentation of the addressee, supplement the interlocutor or object to him), ability to reach a general conclusion. Each thematic series includes a set of problematic tasks, a formal game and a set of specific situations which makes it possible to activate both the professional knowledge of the trainees, and develop professional communication skills, i.e. the ability to evaluate one's own position, the position of the interlocutor and the purpose of communication; ability to fix the position of the addressee of the conversation; ability to formulate and substantiate their own position; ability to oppose (ask questions, supplement, influence the addressee's argumentation, supplement the interlocutor or object to him), ability to reach a general conclusion.

Methodological system for the formation of foreign language communicative competence among postgraduate lawyers was developed proceeding from the theoretical foundations of the research.

The main directions of this technique are:

- use of the communicative-activity method within the framework of the problem approach;

- combination of two methodological tasks in the educational process (improvement of language and the formation of professional communicative competence);

- creation in the process of teaching the language of the specialty of actual motivation for professional communication; 
- allocation as a goal of training activities for the purposeful formation of professional communication skills and abilities;

- gradual formation of professional communication skills and abilities, which implies a gradual increase in the complexity of problematic tasks and the complication of communication tasks of vocational guidance;

- orientation towards practical training, which involves the use of modern forms and methods of teaching, ensuring the readiness for the effective performance of professional activities.

The process of forming a foreign language communicative competence of lawyers is divided into three stages each of which has its own tasks, methods, process content and control of results. At the first, preparatory stage, activities are organized aimed at improving language competence and at developing the ability to build a statement in a given form. At the second stage, a systematic activity is organized to form the ability to assess and maintain one's own position; the ability to assess and fix the position of the interlocutor in communication; ability to oppose. At the third stage, purposeful activities are carried out to improve the skills formed at the first and second stages, as well as work on the formation of the ability to participate in collective work and reach a common conclusion.

Thus, it can be assumed that the optimal method of forming the foreign language communicative competence of lawyers at the postgraduate stage is the active application of the problem-based learning based on the relationship between problem situations and tasks solved in the learning process and the scientific problems of the master student himself.

Problematic tasks determine the purposefulness of postgraduates' speech. Despite the fact that the possibilities for the development of communicative professional skills are limited using these tasks. The use of problematic tasks is justified by the fact that when solving the emphasis is shifted from difficulties of a cognitive nature to the difficulties of the content plan (finding the correct only correct answer) and the expression plan (the need to use adequate language facilities). The stage of work on problematic tasks is focused on the assimilation of new linguistic and theoretical material allows you to organize work on the development of communicative and speech skills using those motivational resources that are contained in this type of task. According to the degree of complexity problematic tasks are designed for the intellectual capabilities of the speaker and for the achieved level of communication and speech skills. Therefore, we can say that in the process of solving problematic problems the teacher manages the process of improving speech skills. The intellectual abilities of postgraduates are developed, communicative professional skills and abilities are improved due to 
the systematic complication of the plan of the content and forms of work (transition to solving specific situations and a business game).

It is obvious that in contrast to work on a specific situation when the task of postgraduates is to search for several possible solutions, this type of work orients students to a specific answer which involves the demonstration of communication and speech skills and knowledge of thematic material. The methodological factors of creating problem situations are based on an orientation towards teaching an unprepared foreign language statement; considering the specifics of the processes of mental activity aimed at overcoming these difficulties.

The work on the formation of foreign language communicative competence is carried out in different languages studied at the postgraduate stage. In this research, this provision is realized on the material of the English language. An introductory step in technology is a series of exercises to teach interpretation. The specificity of the legal system of Great Britain and the United States lies in the fact that the law is of a precedent nature which requires the presence of certain ways of interpretation to be learned. The peculiarities of interpretation within the framework of the legal norms of Great Britain are worked out on theoretical material (Interpretation of the norms of the law).

Since words are an imperfect means of communication many aids have emerged throughout history to help judges interpret laws. The following passage gives an overview of some of the tools. It should be remembered that statutory interpretation is not a scientific process and these "rules" of interpretation are general principles rather than strict norms.

There are several presumptions that govern the judge when he should interpret the Act. There are the presumptions that a certain Act applies to the whole of the United Kingdom, but no more than the sovereign is obligated to that the law is not retroactive that the law cannot be changed. There are certain aids to the judges in addition to the presumptions. In appropriate circumstances the internal means can be considered the title of the law the preamble of the law, the headings of sections and annexes. Marginal footnotes cannot be counted as such as they are not introduced by Parliament. Acts usually contain their own definitions sections. External tutorials are usually not used. The Oxford Dictionary may be referred to as a guide in clarifying the meaning of words used in the Act. The Interpretative Act of 1978 gives specific meaning to the various words and expressions used in the law, unless there is an illegal intent. For example, the Act says that the masculine includes the feminine and the singular includes the plural (section 651), and the time is referenced in GMT (section 9). Preparatory work may be envisaged if it is available to the public and if it unambiguously indicates a specific intention of the legislator (The Interpretation Act, 1980). 
Of particular interest is the ejusdem generis rule (of the same kind). When two or more words are followed by common words, those common words should be read in relation to those specific words. For example, the Betting Act of 1853 prohibited the provision of "a house, office, or other place" for wagering with persons who came there. The question arose as to whether Tatersalls Ring (open annex for bookmakers) fell under this statute. The court applied the ejusdem generis rule that "other places" refers to other indoor habitats and therefore does not include Tatersalls Ring.

Moreover, finally, a few words can be said about the so-called canons (norms) of interpretation. The "restrictive rule" states that when there is no ambiguity then literal or ordinary meaning can be given to words even in spite of the result. An extension to this rule is the "golden rule" of interpretation which can be expressed as it is necessary to strictly adhere to the grammatical and usual meaning of words until this leads to absurdity, contradiction or incompatibility with the rest of the document. In this case the grammatical or ordinary meaning of words can be transformed in order to avoid absurdity, contradiction or incompatibility no more (The Interpretation Act, 1857).

Another rule of interpretation is the "rule of harm". It states that in the event of ambiguity the court can revert to the old law in search of the harm that the Act intended to repair and then can interpret the words of the Act in the light of this knowledge.

Postgraduates are offered situations for interpretation using the canons after a thorough study of the text (traditional non-problematic methods of work are used). Examples of situations for analysis:

1. The law imposed a duty on the company to provide an adequate warning system whenever rail workers did not "repair or relocate" a railway line. The plaintiff's husband died while lubricating and cleaning parts of the railway tracks.

2. The defendant was charged under one Official Secrets Act, which stipulated that no person should "near a prohibited place" interfere with any member of Her Majesty's Air Force from performing his or her duties. George walked into the Defense Department airfield.

3. Section (1) of the Street Torture Act 1959 made it a criminal offense for "an ordinary prostitute to stand or make a sexual offer for a fee in the street or in a public place for the purpose of prostitution." The law also contains the following words: "to ensure that people during their walks in the streets will not be annoyed or made sexual offers for a fee by ordinary prostitutes." The defendant was charged with making a sexual offer for a fee from the balcony of her own use. She filed an appeal. 
A series of problematic tasks is structured in such a way that the level of problematicity of tasks is not the same and depends on how much speech activity is controlled by the teacher. More management guidelines are used then lower the level of problematicity (the requirement to use certain language means or to use the content of a specific previously read text when finding an answer). Therefore, problem tasks in the research process were proposed as the complexity increased. The final stage of each task is the implementation of a written work summarizing the activities of the trainee on the problematic task - essay writing, resume writing and referencing the text using previously entered language and clichés.

Since solving problematic tasks is a systematic work teaching writing consists of a number of cyclical processes. In addition, the teacher must be ready to turn a blind eye to grammatical and spelling errors and openly support the writer not entirely and completely but at the beginning postgraduates must safely submit their creations to academic analysis. Even if the superficial structure of these texts is replete with grammatical errors, we must see deeper, delve into the essence of the ideas embedded in the text. We need to give trainees the opportunity to "bring their thoughts to the surface" and develop their own style for presenting them.

We rely on the principles proposed by Karen L. Greenberg (1988] in accordance with this task. K.L. Greenberg lists ten problems in writing that learners must master:

1) choose and limit the theme;

2) formulate and clarify ideas;

3) organize ideas logically;

4) find and synthesize adequate material to prove ideas;

5) create and evaluate rhetorical moves that are appropriate for the topic and audience;

6) consistently line up paragraphs;

7) use the structure of phrases in accordance with the purpose;

8) use the style in accordance with the purpose;

9) edit what was written in accordance with the conventions of educational writing and literacy requirements;

10) quote or reference in accordance with official requirements

The first series of problematic tasks with a low level of problematicity was aimed at monitoring the assimilation of language and theoretical material in the thematic series.

Postgraduates were offered a list of terms and definitions for the past thematic series and a specific text (texts) was indicated which contains a fundamental answer to the problem posed. Postgraduates were offered a list of terms and definitions for 
the past thematic series and a specific text (texts) was indicated which contains a fundamental answer to the problem task. The work is organized as a traditional quiz involving monologue statements controlled by:

1) correctness of the task solution;

2) use of terms and definitions on the theme;

3) grammatical correctness of the statement

Examples of problematic tasks of the first series:

Assess the following situation and justify your assessment.

Topic: The Law of Armed Conflict

Situation: Syrian Civil War (Ray, 2018)

Terms:

\section{armed conflict}

The terms "war" and "armed conflict" may be used interchangeably to mean any armed conflict of a certain degree between the armed forces of states. The degree of conflict required to constitute a war will vary depending upon the circumstances, the states involved, and other similar factors.

As the Arab Spring swept through the Middle East and North Africa, popular uprisings toppled the authoritarian regimes in Tunisia, Libya, Egypt, and Yemen. In Syria, however, Pres. Bashar al-Assad responded to protests with a combination of political concessions and escalating violence against his own people. The uprising became a civil war that spread violence into neighboring Iraq and provided a fertile breeding ground for militant groups such as the Islamic State in Iraq and the Levant (ISIL, also known as ISIS). Rebel groups seized huge swathes of territory, and the area under government control was reduced to a small strip of land in western Syria. Assad resorted to increasingly desperate and savage measures to maintain power, dropping crude "barrel bombs" on urban populations and using chemical weapons on rebel-controlled territory. As regional powers and Western countries assumed a greater role in the conflict, it seemed inevitable that Assad would be forced from power. Kurdish militias advanced from the Kurdish autonomous region in northern Iraq, and the U.S. conducted air strikes against ISIL forces in both Syria and Iraq. In 2015 Russia, a longtime supporter of the Assad regime, began a bombing campaign in support of Syrian government forces that reversed the tide of the war. Cease-fire agreements failed to stop the violence, and by 2016 it was estimated that 1 in 10 Syrians had been killed or wounded by the fighting. Four million people fled the country, while millions more were internally displaced. At least 470,000 deaths were caused directly or indirectly by the war, and life expectancy at birth experienced a shocking plunge from over 70 years (preconflict) to just 55 years in 2015 . 
During the 19th century, every sovereign state was considered to have the right to wage war. In the 20th century, however, many international agreements, most notably article 2(4) of the UN Charter, have renounced force as a policy and encouraged first resort to peaceful settlement of disputes.

\section{Text (thesis):}

The requirements for a valid act of self-defense were stated by Daniel Webster over 150 years ago in the Caroline case and reaffirmed in Nicaragua v. United States, 1986 ICJ Rep. 14. These requirements apply whether a nation is acting in self-defense against an attack which has already occurred or acting in anticipatory self-defense. First, there must be a «necessity of self-defense, instant, overwhelming, leaving no choice of means, and no moment of deliberations Second, the actions justified by the necessity of self-defense «must be limited by the necessity and kept clearly within it». These two requirements are often referred to as the requirements of necessity and proportionality.

Text (antithesis):

«Older men declare war. But it is youth that must fight and die» (Herbert C. Hoover)

The second series of problematic tasks with an average level of problematicity is focused on knowledge of all the material in the thematic series. The attention of postgraduates is focused on the form of the answer, that is, on the way of reasoning. They are offered a reduced general scheme for constructing an answer, which must be followed:

- formulate the answer to the question;

- explain what this means (use basic vocabulary);

- prove, give arguments. Arguments like "so, because everybody does it" are not taken into account;

- make a conclusion.

\section{The Criminal Prosecution of Terrorists}

Situation: Transnational Terrorist Groups and International Criminal Law

Attacks by network-based transnational terrorist groups cause on average 25,000 deaths every year worldwide, with the law enforcement agencies of some states facing many challenges in bringing those responsible to justice. Despite various attempts to codify the law on transnational terrorism since the 1930s, a crime of transnational terrorism under international law remains contested, reflecting concerns regarding the relative importance of prosecuting members of 
transnational terrorist groups before the International Criminal Court (Brennan, 2019).

\section{Terms:}

\section{Presumption of innocence}

Everyone charged with a penal offence has a right to be presumed innocent until proved guilty according to law in a public trial at which he has had all the guarantees necessary for his defense...

\section{Interpol}

Interpol is an international police organization for helping national police forces to catch criminals. Interpol fights against drug trafficking, smuggling, terrorism and other international crimes, coordinates the police activities of member nations.

\section{Venue}

Venue is a statutory limitation on geographical location of litigation to prevent a plaintiff from suing where it would be burdensome for the defendant to appear and defend.

Text (thesis):

«If your only opportunity is to be equal, then it is not equality». (Margaret Thatcher)

Text (antithesis):

«This is not the end. It is not even the beginning of the end. But it is, perhaps, the end of the beginning». (Winston Churchill)

Use the following words and constructions to solve the problematic problem:

Conjunction: because, in order to, therefore, though, despite, nevertheless, regardless, unless, notwithstanding; but, if, whether, hence, since, rather than, namely, that is

- $\quad$ It is important to compare $\mathrm{X}$ and $\mathrm{Y}$... because $\mathrm{Z}$

- $\quad \mathrm{X}$ is a problem ... because it requires $\mathrm{Y}$.

- $\quad$ This confusion arose ... because of a lack of information / misuse of Y.

- $\quad$ This view is rejected ... because it cannot be verified

- $\quad$ All XX are substitutes ... because they are alternatives

- $\quad$ Our goal is to survey X ... in order to study $Y$.

- $\quad$ In order to measure $X \ldots$ it is crucial to calculate $Y$.

The verbs used in learning objectives or learning outcomes should correspond to the level of thought at which the postgraduates are expected to perform or action. Bloom's Taxonomy classifies critical thinking according to six cognitive leveks of complexity: knowledge, comprehension, application, analysis, synthesis and 
evaluation. The categories are ordered from simple to complex and from concrete to abstract (Anderson, 2001). Here we can measure as the principle of hermeneutics.

Analyzing: Analyze $\bullet$ Assume $\bullet$ Categorize $\bullet$ Classify $\bullet$ Compare $\bullet$ Conclusion

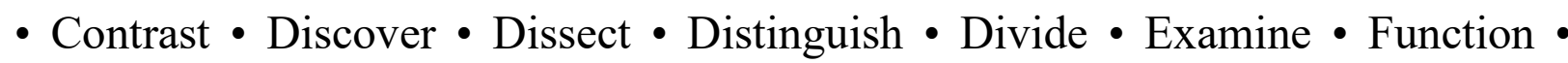
Inference $\bullet$ Inspect $\bullet$ List $\bullet$ Motive $\bullet$ Relationships $\bullet$ Simplify $\bullet$ Survey $\bullet$ Take part in $\bullet$ Test for $\bullet$ Theme

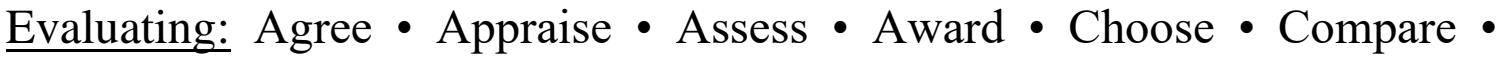
Conclude $\bullet$ Criteria $\bullet$ Criticize $\cdot$ Decide $\bullet$ Deduct $\bullet$ Defend $\bullet$ Determine $\bullet$ Disprove - Estimate $\bullet$ Evaluate $\bullet$ Explain $\bullet$ Importance $\bullet$ Influence $\bullet$ Interpret $\bullet$ Judge $\bullet$ Justify - Mark $\bullet$ Measure $\bullet$ Opinion $\bullet$ Perceive $\bullet$ Prioritize $\bullet$ Prove $\bullet$ Rate $\bullet$ Recommend Rule on $\bullet$ Select $\bullet$ Support $\bullet$ Value

Creating: Adapt $\bullet$ Build $\bullet$ Change $\cdot$ Choose $\cdot$ Combine $\cdot$ Compile $\bullet$ Compose - Construct $\bullet$ Create $\bullet$ Delete $\bullet$ Design $\bullet$ Develop $\bullet$ Discuss $\bullet$ Elaborate $\cdot$ Estimate $\bullet$ Formulate $\bullet$ Happen $\bullet$ Imagine $\bullet$ Improve $\bullet$ Invent $\bullet$ Make up $\bullet$ Maximize $\bullet$ Minimize - Modify $\bullet$ Original $\bullet$ Originate $\cdot$ Plan $\bullet$ Predict $\cdot$ Propose $\cdot$ Solution $\cdot$ Solve $\bullet$ Suppose $\bullet$ Test $\bullet$ Theory

It should be noted that in the first lessons when solving problematic tasks of the second level of complexity a typical mistake in the answers of postgraduates was the inability to build a logical statement. So, after formulating the answer to the question, explanation and reasoning were replaced by examples.

The work is carried out in groups; preparation time is limited to 5-7 minutes. Master students are allowed to use the basic textbook due to which conditions are created for the "involuntary" repetition of the past material in a rather extreme educational situation.

Controlled by:

- correctness of solving the problematic task;

- form of constructing the statement;

- language competence.

The third series of problematic tasks is considered by us as tasks with a high level of problematicity. The main emphasis when planning tasks in this series is placed on the development of the thinking and cognitive abilities of postgraduates. Completing the tasks of the third series involves a detailed understanding of the material covered.

Postgraduates are invited to come up with a problematic task on a thematic series themselves. The work is carried out in groups (teams). The effectiveness of this type of tasks lies in the fact that they allow you to assess the degree of comprehension of the material the ability to highlight and anticipate the problem 
based on the degree of controversy of the legal norms presented in the section under study.

The logical conclusion of the work is the promotion of a number of the best topics proposed by postgraduates as a result of the third series of problematic tasks for discussion in the group.

As this research has shown working with problematic tasks allows to achieve the following methodological tasks:

- improve communication and speech skills including the ability to plan an utterance, choose the most adequate means of verbal formulation of an utterance;

- to increase the level of assimilation of new lexical material, remember its linguistic forms, meanings, methods of use in a certain context;

- systematize knowledge on the topic;

- transfer knowledge from passive to active stock.

The results of the research showed that the methods of problem learning used also allow involuntary memorization due to the fact that when solving a problem task postgraduates turn to the studied texts compare a familiar and a new context determine the peculiarities of the use of lexical and terminological units in different contexts, comprehend their meaning and content (Passov, 1983).

The positive results of the experiment show that when teaching communicative professional skills combining multi-type tasks within the framework of the problematic approach allows purposefully and systematically to achieve the goals set within the interdisciplinary approach.

The specificity of language teaching in a sense limits the possibilities of problem learning and forces the use of combined methods, i.e. the adequacy of the application of one method or another is always determined by the focus of a particular stage of training on the development of language speech or communication skills. Therefore, in our case we consider it appropriate and most effective to use 1) the method of professionally oriented teaching and 2) the method of case study.

Method of professionally oriented teaching. In the process of implementing professionally oriented teaching of a foreign language in an educational environment, there should be an exchange of relevant professionally significant information informing about the current level of development of professional and industrial culture containing relevant figures and facts. From the information of the historical plan it is recommended to include the information that helps to better understand and assimilate the features of modern professional and industrial culture. The use of obsolete information is unacceptable since it can lead to the formation 
of an incorrect distorted idea of the professional and industrial reality of the country of the target language a decrease in interest in the information received.

The source of the selection of texts as indicated above should be a synthesis of authentic disciplinary publications. It is proposed to divide the array based on purpose that is the purpose of creating and using the publications included in it. Firstly, educational literature and secondly, business documents, materials of scientific and technical conferences, brochures, magazine publications, annual reference publications. Guided by the proposed criteria we concluded that the use of only educational literature seems inappropriate. First, the criterion of repeatability and variability of subject content is not satisfied: why read in English what can be read in the native language. Second, the criterion of modernity and contemporary historicism is not fully observed. In addition, it is unlikely that in the future students with such knowledge will turn to such sources and if they do it will be easier to obtain information from sources in their native language. In our opinion, another group of sources has much greater potential in terms of the criteria put forward materials from seminars, scientific and technical conferences, magazines, brochures. In order to confirm our assumption, we turned to a typical genre of such publications, an article.

So, let us analyze the genre of the article in terms of the proposed criteria.

First, as already indicated a scientific article acts as the primary source of information about scientific knowledge is at the very forefront of research. This means that the genre of the article will conform to a high degree to the criterion of modernity and actual historicism the observance of which is extremely important for the formation of the trainees' ideas about the current level of development of a different professional production culture.

Secondly, it is possible to comply with the criteria for variability and repeatability of subject content based on the texts of articles borrowed from an authentic publication. As it was established the professionally oriented disciplines taught in the course of study serve for the trainees, basically, as a source of knowledge of two types: universal and reflecting the specifics of the professional and industrial culture of their native country. Authentic sources fill the information vacuum if the subject concepts contained in them correspond to the level of subject competence of the learners.

Thirdly, in order to identify the texts of authentic articles corresponding in their subject characteristics to the level of subject competence of the trainees a substantive analysis of the texts of articles taken from authentic periodicals presented in the textbooks "International Terrorism", "Drugs" (Uteubayeva, 2002, 2013) and "Professionally oriented English for lawyers" as well as articles published in collections of scientific and practical conferences. As a result, the types 
of authentic texts were identified that satisfy the named criteria. These included: 1) texts of articles, as a rule of a large volume (at least 1500 words); 2) texts of brief informational articles (at least 500 words) of similar content. Let us present the results in more detail.

By the nature of the tasks being solved the texts of articles functioning in the scientific sphere of communication are legal. The volume and combination vary depending on the purpose of the speech utterance. The general structure of texts of large articles is shown schematically in Figure 15.

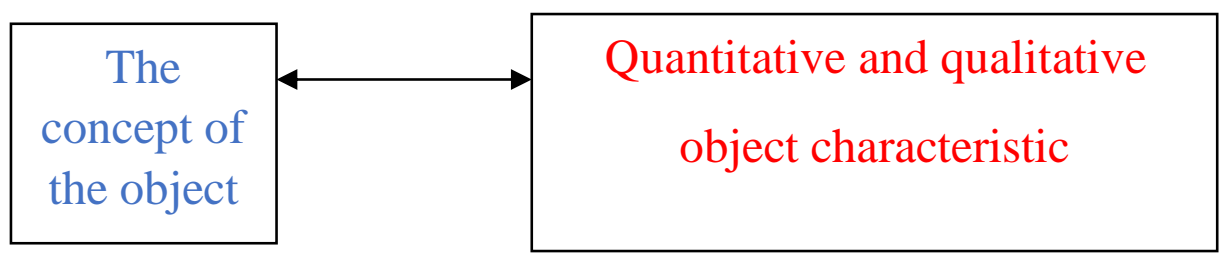

Fig. 15. Typical structure of articles of large volume

The texts of short informational articles are distinguished by the concise presentation of information.

As one of the factors that affect the use in the process of learning a foreign language selected text, perform their speech characteristics. Therefore, it is also important to study the speech organization of a scientific article and the degree of its correlation with the nature of the speech competence of postgraduates. We mean a certain level of proficiency in reading skills and abilities by speech competence.

The most important speech characteristic of a text is its logical and compositional structure. Proceeding from this that in principle, taking into account the specific purpose of the article genre we conclude that description and communication are the main tasks of scientific articles. The communicative purpose of both the description and the message is to present sufficiently complete and adequate information about the object (s) or event (s) of the external world with the difference that the narrative type of information conveys information that is characterized by the sign of dynamism and the descriptive type the sign of static (Grishina, 1982). The communicative purpose of scientific articles is fully consistent with the activities of postgraduates, as well as the empirical nature of this activity at a certain stage of training.

Descriptive texts are the easiest to understand. According to observations creating the correct direction of thought based on knowledge of the topic provides a reliable basis for understanding that omission of any small detail can lead to some incompleteness of understanding but does not lead to a misunderstanding of the entire description as a whole since the theme itself largely determines the content of the description (Milashkina, 1988). The dynamic nature of the message moreover 
often accompanied by descriptions can complicate the understanding of information lead to incorrect conclusions about the cause-and-effect, temporal and other relationships of events. However, messages with developing action according to the observations of many researchers and our own are of greater interest and, therefore a greater concentration of effort and attention which positively affects the level of understanding.

The legal description and message are always substantive and objective they are characterized by concreteness and brevity. They strive to give the recipient of information a specific idea of an object / subject or event, therefore their integral component is specific data. The text definitely gives digital data and an overview indicates dates, etc., which serves as a means of correlating abstract knowledge with an object of reality a means of displaying not only the external but most valuable the internal structure of an object given in the subject plan of the text. Specific data summarize, systematize, illustrate scientific knowledge, optimizing the understanding of the text up to $100 \%$ in completeness (Shergina, 1983).

The elements of the text of a scientific article are ordered in such a way that all relatively independent units that have scientific and informational value which are part of it are integrated into a single textual whole both at the informational and structural levels. Informational integration provides the content unity of the scientific text and is focused on the full disclosure of its content. Structural integration provides the existence of a scientific text as a linguistic whole, which is a hierarchy of linguistic units (Chulkova, 1980). It is interesting that the information of scientific articles is almost completely encoded according to the external structure and therefore is explicit. This undoubtedly has a positive effect on the level of understanding of the message especially if we take into account the data obtained on the initial level of the speech competence of trainees. When determining the initial level of speech competence, it seemed expedient to rely on the level of formation of speech skills that are important for the implementation of the main types of professionally oriented reading: informative and reference.

T.S. Serova (1989) identifies and describes 7 skills of reference and 9 skills of informative professional-oriented reading. All skills of reference reading are based on the ability to determine the topic of the source by key concepts, referents, and on this basis:

1) carry out a general orientation in the subject of the source, its referents and refer it to a specific objective reality;

2) carry out orientation in the source, to distinguish their themes and refer to objective reality;

3) carry out orientation in sources of different genres and content;

4) carry out a targeted search for a source according to the given text 
referents;

5) carry out targeted search and distinguish between topics, text referents;

6) carry out the selection and concretization of all referents, referring the source to objective reality;

7) distinguish between topics, clarify and generalize them in order to refer to a specific reality.

The main skill of the informative type of professionally oriented reading is the extraction of information on a topic in order to:

1) compare it with your research theme;

2) evaluate it;

3 ) change the volume and quality of your information;

4) reproductively formulate and record in order to gain individual experience;

5) plan, regulate and organize all types of its activities;

6) productively formulate and record information and create your new text (new information);

7) exchange new information with communication partners;

8) when exchanging it, change the motives of the partners' activities, induce them to other types of activities

Given the basic elements allocated abilities in determining the level of foreign language speech competence trainees taken into account the level of formation:

1) ability to determine the topic by key concepts and as a special case predict the theme and the main content of the source by the title which has a key position in the communication of scientific information;

2) ability to extract factual information from the source.

The skills listed above naturally involve the parallel development of writing skills. Learning to write at the school and university stages involves mastering spelling and performing training (language) exercises in writing. Writing training includes speech exercises for teaching writing a written message, writing and speaking exercises in working with printed text due to the process of reading, listening and oral communication.

However, at the postgraduate stage of study it is assumed that the required level of written proficiency at least corresponds to the European level B1 of advanced language proficiency. The goal of optimizing the written component of the foreign language professional communicative competence of a master law student is to achieve the European level B2 of advanced proficiency which significantly changes the teacher's approaches to the form and content of written assignments.

The main emphasis in teaching writing at this stage should be done on helping 
postgraduates to express their thoughts on paper. It should be done logically, reasonably and concisely to develop an author's voice within the framework of their scientific problems, and this voice can be cut through only due to nothing unrestrained writing process, when the writer allows himself to take risks and experiment with the word (Randall, 2002). It is these parameters that the teacher should take into account when definition of subject and focus of written work.

On the other hand, many teachers and even postgraduates, accept the traditional hierarchical structure of the relationship teacher - contributor of knowledge and postgraduate is a vessel for knowledge (Freire, 1972). Therefore, the freedom of discourse is limited as it is limited in any hierarchical and especially the academic community which does not contribute to free self-expression and the development of the author's voice of the trainees. We believe that the optimal formation of the foreign language professional communicative competence of postgraduates is possible only when the initial level of language proficiency the student's motivation and the scope of his scientific interests are taken into account.

\section{Use of case study}

The case study method began to be used first in management education at the School of Business, Harvard University in 1908 which is considered the year of the appearance of this method. For almost a century of application of this method many theoretical and practical guidelines for working with this method have appeared (Naumov, 2020).

Today it has become generally accepted that this method is most conducive to the development of postgraduates' inventiveness the ability to solve problems taking into account specific conditions and in the presence of information. The case study method develops the ability to analyze and diagnose problems. With its help postgraduates develop such qualifications as the ability to clearly formulate and express their position, ability to communicate, discuss, perceive and evaluate information received in verbal form. Finally, this method contributes to the development of trainees' sense of self-confidence, helps to overcome excessive shyness and excessive self-confidence" (The Interpretation Act, 1996).

Among the advantages of this method are the following:

1) specific situations tie learning to reality analyzing the situation postgraduates get acquainted with what happened in real life and not with an abstract theoretical generalization.

2) working with a specific situation leaves the trainee freedom in finding ways to analyze, identify and solve the problem. The educational process is carried out in the form of a creative search.

3) when analyzing a specific situation, postgraduates take an active position. 
At the same time, they actively interact with each other which as it were imitates real communication dependencies that arise between people in the process of their joint activities.

4) in the process of analyzing a specific situation postgraduate themselves come to the creation of a certain generalized knowledge that has a conceptual character.

Situation analysis requires trainees to pose questions for themselves and look for ways to answer them while generalizing for themselves the idea of methods for solving problems (Uteubayeva, 1977).

The effectiveness of the application of this method in law faculties has not decreased moreover it became obvious that the use of this method in teaching legal disciplines allows not only mastering a large amount of information but also comprehending it training it in situations that imitate real life.

Considering that teaching by practical examples as a way of development is considered one of the best methods for the development of analytical and critical thinking as well as creativity, it is proposed to more widely implement such a teaching method as case study in special educational institutions. During the process we invite teachers to develop descriptions of certain non-standard situations which the law enforcement agencies actually encountered in their activities in order for the postgraduates to get acquainted with the problem and find a solution to the case independently and in the course of collective discussion. It is impossible to learn investigation and judicial practices from existing textbooks. An alternative to textbooks was interviews with leading lawyers' practitioners and detailed reports (descriptions of situations) written on their basis on how the specialists solved a particular situation as well as on the factors affecting their activities. One of the most effective tools for improving educational activities is the functioning of the Courtroom in special educational institutions. The purpose of the activity of such a training hall will be to provide postgraduate with professional skills and abilities in conducting practical classes in disciplines and special courses related to criminal, civil, arbitration proceedings, labor disputes, etc.

Considering the functions of the CS as a component of the content we find in the methodological literature an assumption to consider the situation as the main core around which the material is organized. This assumption is substantiated as follows in the works of E.I. Passov (1983) and his students that the situation sharply narrows the choice of speech units precisely because it is a system of relations that a person in the process of solving operational problems, structures according to the goal which directs his activity. This streamlines the selection of speech material for each situation. In the domestic methodological school of foreign language education there is not only a tendency to expand the boundaries of the professional and 
production communicative sphere but also focused attention to the category of professional situation to the specifics of its competent composition in the conditions of professionally-based subject content to the creation their variable modeling to ensure flexibility of context-based skills (Tepley, 2014).

The problem that the trainees must solve as a result of the general group discussion is not constructed by the teacher on the basis of the material passed and subject to consolidation. The specificity of a particular situation lies in the fact that it contains a description of real facts and events and the description does not contain, in principle evaluative moments that could guide the creative search of the postgraduates. In addition, specific situations used in the framework of the problem approach can be focused on illustrating the theoretical material covered can be aimed at teaching decision making or learning to analyze and systematize information (Belyaev, 1997).

Methodological developments for training based on specific situations recommend analyzing situations in three stages.

The classical scheme for parsing the case study method assumes that at the first stage, the trainees individually study the text of the situation in detail independently highlight the problem, look for ways to solve it and prepare for a group discussion.

The second stage consists in the fact that trainees in small groups discuss the highlighted problem and ways to solve it thereby expanding the vision of the situation and the content of the problem.

The third stage is a group discussion which is conducted under the guidance of a teacher who acts as the organizer of the discussion. The purpose of the third stage is a meaningful analysis of the case study method fixing several possible ways to solve the problem, focusing on the theoretical substantiation of the solutions found on the conceptual solution of the problem. The peculiarity and complexity of the third stage is that the teacher must organize the discussion in such a way as to involve the maximum number of participants in it. In addition, the teacher must ensure, firstly, to correctly distribute the roles of the participants taking into account the level of training of the trainees and secondly, to regulate the course of the discussion in such a way as to direct it in the right direction. We can say that the effectiveness of the third stage largely depends on the skill of the teacher (Belyaev, 1997).

Each CS contains questions that correspond to the problem discussed in the text, but the following are distinguished as the general format of the questions:

a) What problem did the company / individual face?

b) What way out or ways out of the situation would you recommend to the company / individual? 
c) What are your recommendations? Make a convincing case for your recommendations.

The analysis of the case study in the classical version of this approach is preceded by practical exercises that contribute to informative saturation and preparation for the search for conceptual ways to solve the problem when analyzing the case. The knowledge of the postgraduates is updated if the situation is illustrative then its analysis and discussion automatically give the master student a lot of additional information.

The use of the case study method in the classroom in the language of the specialty has its own specific features. On the one hand the method carries a strong motivational charge orienting trainee to mastering special professional communication knowledge, skills and abilities. The application of this method allows us to say that with this approach to teaching the language becomes not the goal of teaching but a means by which postgraduates learn to communicate and in addition, study the features of the legal system of the country of the target language. On the other hand, the teacher solves the problem of improving the level of postgraduates' proficiency in the studied language; the program for the course of the language of the specialty contains a certain set of lexical units and grammatical phenomena subject to assimilation and control. The combination of the listed tasks in the course on the language of the specialty required the adaptation of this method and determined the specifics of its application in classroom lessons in the process of conducting experimental training in professional communication skills and abilities.

The analysis of the CS in the target language is preceded by work on reading the corpus of texts on the selected topic. Thus, the analysis of the Constitutional Court on the topic "The features of the English legal system" is preceded by a thematic series of texts telling about the characteristics of the English legal system, historical path of its development, specifics of civil law. Work with the text carried out using synthesizing methods based on the corresponding maps of conceptual fields allows you to systematize lexical and grammatical material. A series of language exercises focused on the development of language competence is accompanied by a series of communication exercises aimed at developing communicative-speech competence. (Uteubayeva, 2005). Thorough study of the lexical, grammatical, and importantly theoretical material presented in the text is the key to the success and effectiveness of the use of CS. It should be emphasized that the work with the texts was carried out according to a single program within the framework of traditional classes in the language of the specialty both in the experimental and in the control groups. A special course on teaching professional communication skills was "built on" over the postgraduate's program by allocating 
hours of classroom lessons from the total volume intended for mastering the thematic series. In the control groups the last four hours were allocated to traditional forms: preparation and referencing control tests.

PBL instruction in the classrooms consisted of:

1. Module 1: Warm-up and vocabulary. The instructor taught vocabulary and new content knowledge by asking questions. The teacher provided postgraduates with opportunities to discuss within a small group. The postgraduates responded to the questions and received evaluation and cognitive feedback from the teacher.

2. Module 2: Essay structure. The instructor divided postgraduates into three large groups. They were requested to discuss the main idea and structure of the text. After the discussion a representative of each group presented their findings.

3. Module 3: Writing. The instructor provided postgraduates with opportunities to discuss the structure and writing techniques of corresponding reading materials. A representative of each group presented their ideas to the whole class and received feedback from the instructor. The instructor introduced one or two writing samples and evaluated their strengths and weaknesses. In the end, the teacher summarized writing techniques and assigned postgraduates a writing template for practicing writing after class.

Consider a number of interactive teaching methods, the introduction of which will help achieve this goal - increasing the efficiency of the educational process and achieving results for all students.

\section{Group discussion}

Discussions in groups are usually conducted on a specific topic and aimed at finding the right solution and achieving better mutual understanding. Group discussions contribute to a better assimilation of the studied material. At the first stage of the group discussion, postgraduates are given an assignment for a certain time, during which they must prepare a reasoned, detailed answer.

The teacher can set specific rules for conducting group discussions:

- implementation of an algorithm for identifying a consensus opinion;

- $\quad$ appointing a leader to guide the group discussion.

At the second stage, such discussions with the teacher lead to group decisions. A type of group discussion is the "Round Table", the purpose of which is to exchange information about the problems and their own understanding of the issue in order to familiarize themselves with the experience and achievements in this area. 
Training. Training is a form of interactive learning, the purpose of which is to develop interpersonal skills and professional behavior in communication. The advantage of the training is that all participants are actively involved in the learning process.

Training requirements:

- $\quad$ the optimal number of participants is 15-20 people, according to the number of participants in the audience, which contributes to the active cooperation of its members;

- familiarizing participants with the objectives of this lesson at the beginning of the training;

- conducting at the first lesson the training-exercise "acquaintance" and the adoption of the "agreement", that is, the rules of the group work;

- creating a friendly atmosphere of trust and maintaining it throughout the training;

- inclusion of all participants in active work during the training;

- $\quad$ respect for the feelings and opinions of each participant;

- technical support of the educational process;

- $\quad$ effective combination of theory and interactive exercises;

- compulsory summing up of the training results after the completion of the training.

The teacher conducting the training must have psychological and pedagogical knowledge and be able to skillfully use it in the learning process, know the methods of obtaining information, collect and present it to participants, influence their behavior and their relationships.

Discussion. In the process of discussion, postgraduates must discuss problems publicly or freely exchange knowledge, opinions, ideas on controversial issues. Its essential feature is the combination of dialogue-discussion and disputecollision of different points of view and positions. Discussion is a student -centered learning. It is characterized by active interaction of postgraduates with each other and intensive, person-centered learning on the part of the teacher. The merit of a discussion is that it shows how well the group understands the issue. Any discussion usually takes place in three stages:

1. Formulation of the problem;

2. Solution to this problem;

3. Summing up.

The first stage is the adaptation of the participants to each other, through which it is possible to formulate the problem, the goals of the meeting, the rules and 
rules of the debate. The second stage involves the participants speaking, their answers to questions, collecting as many ideas and proposals as possible, suppressing the personal ambitions of the participants in the discussion and deviations from the discussion of topics by the teacher. And the third stage is the analysis of the results of the discussion, agreement of opinions and views, joint formulation of decisions and their adoption. During the discussion, students can either complement each other or oppose each other. The type of discussion chosen by the teacher, depending on the task assigned to him, can be a combination of different types of discussions (classical discussion, express discussion, text discussion, problem discussion, role play, round table). The merit of the discussion is that the discussion for a short period of time (and the volume of hours for postgraduates is limited) allows the teacher to model real problems, develop the ability of postgraduates to listen and share their thoughts with other participants, interact and analyze a real situation, separate the important from unimportant. Thus, the discussion makes it possible to understand and evaluate the diversity of existing views on any problem, to conduct a comprehensive analysis of each of them, after hearing the individual opinion of each participant in the discussion on this topic. This form of work with postgraduates is very important and productive, since during the discussion they acquire the communication skills they need for their further professional development.

Brainstorming method. This is a fairly popular method for solving problems by stimulating creativity. According to this methodology, the teacher invites a group of postgraduates to give as many answers to the question as possible. Brainstorming takes place in three stages (Safonova, 2015). Brainstorming consists of implementing a method of gathering together whereby a group tries to find a solution to a problem by pooling all the ideas which are presented at the same meeting (Riziab et al., 2013). The brainstorming method was founded in 1938 when Alex F. Osborn applied organized idea-seeking. The brainstorming session has rules which serve as guides for knowledge and are not restricted at all. (Harir Foroush \& Sadeghi, 2010).

\section{The rules are as follows:}

1. Withhold criticism: While expressing their ideas, none of the members should criticize an idea presented by others, even if it is totally absurd. At the end of the session opinions about different ideas will be surveyed.

2. Flying in dreams: this means that during the session, members should put aside all restrictions of thinking. In fact, for creative thinking, one should trespass ordinary logic and usual red lines. 
3. Focus on quantity: The more the number of ideas, the easier their classification.

4. Documentation: each idea, however banal, should be recorded and then exposes to others to see it.

5. Combine and improve ideas: Before ideas are evaluated, they should be completed. Through this, the ideas which were recklessly presented are now revised and completed.

Stages of Brainstorming:

Stage one: introducing the brainstorming rules.

Stage two: stating the subject or problem.

Stage three: expressing ideas.

Stage four: exhibiting ideas for combination and improvement.

Stage five: evaluating ideas.

\section{Research question:}

The current study aims to answer the following question:

Is the educational achievement of the students who are trained through brainstorming higher than the achievement of those who are trained via explanatory method? (Harir Foroush \& Sadeghi, 2010)

So, at the first stage, a problem is posed to the group. Participants make guesses one after another in an accurate and concise manner, the teacher writes down their ideas on the board or poster without any comments. The second stage is to discuss these ideas and exchange points of view. At this stage, the group needs to find a way to use or improve their ideas. At the third stage, the group presents the results of the work. For Brainstorming, you can divide the participants into several groups:

- generators of ideas that formulate various proposals for solving a problem;

- critics who try to find the negative in the proposed ideas;

- analysts linking the developed proposals with real conditions, considering critical comments, etc.

This method is one the modern methods of teaching and learning. It begins with forming several groups, division of duties, and active participation of postgraduates. Taking to account the current research, the positive effect of this method on the educational achievement of postgraduates is confirmed.

Problem learning. In the context of problem-based learning, not only the problem of education or problematic task is important, but also the skillful presentation of questions by the teacher. Questions in the process of organizing the 
activities of postgraduates will help teach them to reproduce the information received from memory and to perform reproductive actions. As a result, this will stimulate the creative thinking of trainees, allowing them to discover and acquire new knowledge, skills and abilities.

Basic principles of working in an interactive lesson:

- all participants are the same regardless of age, social status, experience and place of work;

- each participant has the right to express his opinion on each issue;

- there is no room for direct criticism of the personality (you can only criticize the idea itself).

Once again, we emphasize that when analyzing the CS, the teacher focuses the attention of postgraduates and directs them to demonstrate professional communication skills. Language and speech errors made by trainees during presentations are analyzed in a separate lesson, held after the analysis of the CS.

In the first chapter we highlight the requirements for the communicative competence of a lawyer. In addition, we identified standard criteria for general rules of behavior and communication, which according to experts should be paid attention to. Among these standard requirements there were techniques that are honed through special training and purposeful training, for example, showing selfconfidence, reliability, benevolence, solidity; ability to instill in a partner a sense of greater security, freeing him from fear, feelings of hopelessness or uncertainty, motivation to take the right action, etc. Analysis of the discussion in the future allows drawing the attention of postgraduates to errors of this kind.

The situation is proposed considering the topics of scientific research in civil proceedings (The Interpretation Act, 1977).

Facts: The plaintiffs owned a house adjacent to a cricket court. Cricket was played on this site long before the house was built. The plaintiffs complained about the damage caused by the cricket balls and that they were unable to enjoy their property. They sued the cricket club for private malice, seeking damages (a common law remedy) and an injunction (a court of justice remedy) for playing cricket on the court. The cricket club argued that it did everything it could to prevent balls from entering the plaintiffs' garden, including erecting a 15 -foot fence.

The court decided that the cricket club was guilty before the plaintiffs for private malignity. They were awarded compensation for damages, but the appellate court rejected the injunction to play cricket. Lord Denning, Head of the Court of Appeal: "The court must remember that it has a duty to take the public interest into account when it decides whether to exercise equity jurisdiction and to issue an injunction. When an injunction would result in an end to the game of cricket on a court that has been played for 70 years or so, it must be borne in mind that the public 
interest must prevail over the troubles of private owners who have been prevented from enjoying themselves in silence and peace, home and garden, while playing cricket."

Do you see a difference in the court's approach to common law versus equity court remedies? To obtain redress, the plaintiffs only had to prove that the defendants were guilty of malignity. Having proved this, the court did not have the power to deny a common law remedy (although the judges must determine the amount that the defendants must pay). Conversely, since the court's remedies for fairness are discretionary the court could refuse the order because it considered that it would be unfair and in bad faith to do so.

This stage of work is characterized by the fact that it allows to actively involve into the work of postgraduates with weaker language training. It is important that the analysis of the COP allows transforming the role of the teacher in the teaching process from didactic (when the main thing is to give "new knowledge" and check its assimilation by the students) to motivational (the main thing is the formation of specific skills of a lawyer the ability for self-development, decision-making, leadership, communication skills) (Uteubayeva, Kultanova \& Pastushenko, 2012).

Here, at the first lesson on the CS postgraduates are presented with the goals and objectives of this stage it is shown how it is necessary to work with the content of the CS. What are the main structures in the text that should be highlighted so that the identification of the problem, its analysis and the subsequent argumentation of the proposed solutions meet the requirements for this type of occupation, and not turn into an ordinary educational polylogue, the purpose of which is to demonstrate language and speech skills. The most important thing in the first lesson is to orient the trainees to the fact that this type of work is the development, first of all, of communicative professional skills.

It is necessary to present to postgraduates an algorithm for working on a situation, to emphasize the admissibility of not one, but several optimal "solutions" of the situation.

Algorithm for working on the CS:

1. Individual study of the situation in the text by postgraduates (homework);

2. Control detailed understanding of the text (translation, frontal survey) and knowledge of the material.

The following questions can be suggested to check the understanding of the problem:

- Who is the character of this situation?

- Whose interests are represented?

- What is the main problem discussed in this situation?

- What section of law illustrates this situation? 
The duration of this stage depends on the complexity and size of the CS. Experience shows that at this stage it is advisable to involve postgraduates with weaker language training in the work. Since the questions require non-detailed answers already contained or implied in the text and those less prepared have a good opportunity to activate their speech skills and abilities which creates the effect of general participation in the discussion, increases their self-esteem and activates motivational resources.

3. Introductory word of the teacher, fixing questions to the CS, for example,

- What problem did the company / individual face?

- What way out or ways out of the situation would you recommend to the company / individual?

- What are your recommendations? Make a convincing case for your recommendations.

4. Distribution of trainees in small groups (no more than 3-5 people in each group). The composition of the groups changes at each lesson which allows master students to develop communication skills, to perform in different roles in different groups.

5. Work of listeners in a small group according to a typical scheme, choice of a "speaker". In order not to create a situation when the same speakers act as speakers in the classroom (work experience shows, as a rule, this role is willingly "captured" by master students who have the qualities of a leader not always with stronger language training therefore it is advisable to appoint speakers).

An important point in the work on the CS according to the classical scheme is the isolation of groups that is, members of one group should not hear the discussion of another group, which creates a picture of the work of independent experts.

6. Discussion in small groups is accompanied by the fixation (on sheets of large-format paper) of the decisions made and argumentation in the form of diagrams or in the form of a concise presentation of the discussion. It is advisable to appoint a master student in advance who will record the course of the discussion on paper. Those who have weaker training are appointed as "secretaries". Experience shows that the entire group closely monitors the content being recorded correcting mistakes made by the "secretary" during the recording. Thus, in the process of fixing the discussion, grammatical structures, spelling are trained and, no less important summarizing skills are developed which are an integral part of the professional communicative competence of a lawyer.

7. Presentation of the "solutions" of each small group. The presentation should meet the general requirements for public speaking: contain an introduction, main content and a short summary of the proposed solution. An obligatory 
requirement for the content of the presentation: it should not be "everyday" in nature, it should be made from the standpoint of an appropriate theoretical approach. The substantive part certainly includes the analysis of situations and the argumentation of the decision made using previously studied material on the topic.

8. General discussion, questions, presentations from the field. At this stage, it is very important that the found ways of solving the problem that is the core of the situation are different. Only in this case a lively discussion arises questions from members of other groups to the speaker. The result of the general discussion is finding the optimal solution to the problem under discussion. The teacher is in this case the direct organizer of the discussion; he not only guides the discussion, but also, if

9. Moderator's speech, his analysis of the situation. At this stage the moderator gives his own analysis of the situation thereby demonstrating presentation techniques. In addition, the teacher's presentation aims not only to analyze mistakes made or to celebrate successful performances. From the point of view of language training the teacher's presentation is a good repetition of new lexical and grammatical material. As mentioned above, the language mistakes of postgraduates understand in a specially designated lesson.

Subsequent classes on the analysis of the CS are carried out according to the standard scheme, but with a full-fledged second stage (discussion in small groups) with a general group discussion. Taking into account the specific features of the work on the CS, which consists in the fact that there are restrictions on the volume of the situation determined by the level of language proficiency mini-CSs are used in the future. For example, after analyzing the typical sample of work on the CS, given above, mini-situations for working in the classroom in the same thematic series are also proposed: read the facts of the next case and prepare a short judgment based on your knowledge of the maxims (canons) of justice. Discuss your decision with other members of your group and be ready to justify it by referring to the canons.

The next stage of the formation of professional communication skills in the language classes in the conditions of master's degree preparation we refer to the classroom studies conducted according to the scheme of a formal game conventionally called "Discussion". This type of work is the most complexly organized, includes tasks directly aimed at the formation and development of professional communication skills. It should be noted that it is the complexity of the forms of mental work, and not the linguistic or informative richness that makes it possible to single out this type of work in a separate stage. In addition, the business game provides for the operation of theoretical and linguistic material studied in previous classes and presupposes the obligatory appeal of postgraduates to new 
texts on the same subject. The theme is determined taking into account the research work of the students themselves. To find the correct and convincing detailed argumentation during the game postgraduates turn to other sources of information. Thus, because of an experiment carried out at the Karaganda Law Institute of the Ministry of Internal Affairs of the Republic of Kazakhstan, Karaganda State University named after E.A.Buketov, it was recorded that $70 \%$ of postgraduates in search of missing information, turn to materials posted in Internet. That is, they carry out cognitive and search activities; they independently work through numerous texts (viewing and search reading). Such regular work initiated (but not specifically supervised and not required) by the teacher is built on the use of cognitive motives of the students activates their interest in studying the course and creates conditions for competition in learning. All of these points have a positive effect on the improvement of the linguistic and communicative competence of postgraduates noticeably affect the level of argumentation and evidence and allow us to speak of a higher level of comprehension and study of problematic tasks.

Taking into account the goals and objectives of the course in the language of the specialty which were the basis of the experiment in this research we turn, first of all to the consideration of those opportunities that develop professional communication skills. As the research of G.A. Bekmukhamedova four levels of communicative competence in the polylogue can be distinguished:

- skills of the first level - the ability to compare your own point of view with the point of view of the interlocutor;

- skill of the second level - the ability to request information, clarify and supplement the speech of the interlocutor;

- skill of the third level - the ability to keep the purpose and topic of discussion, as well as leadership in the discussion;

- skill of the fourth level - the ability to formulate the essence of the problem and convince the interlocutor that he is right.

The formal game takes place according to a certain scheme and allows you to work through the studied material, gives effective results since postgraduates are offered creative problematic tasks that require mental tension their activity is activated and the emotional mood rises (Figure 16). 


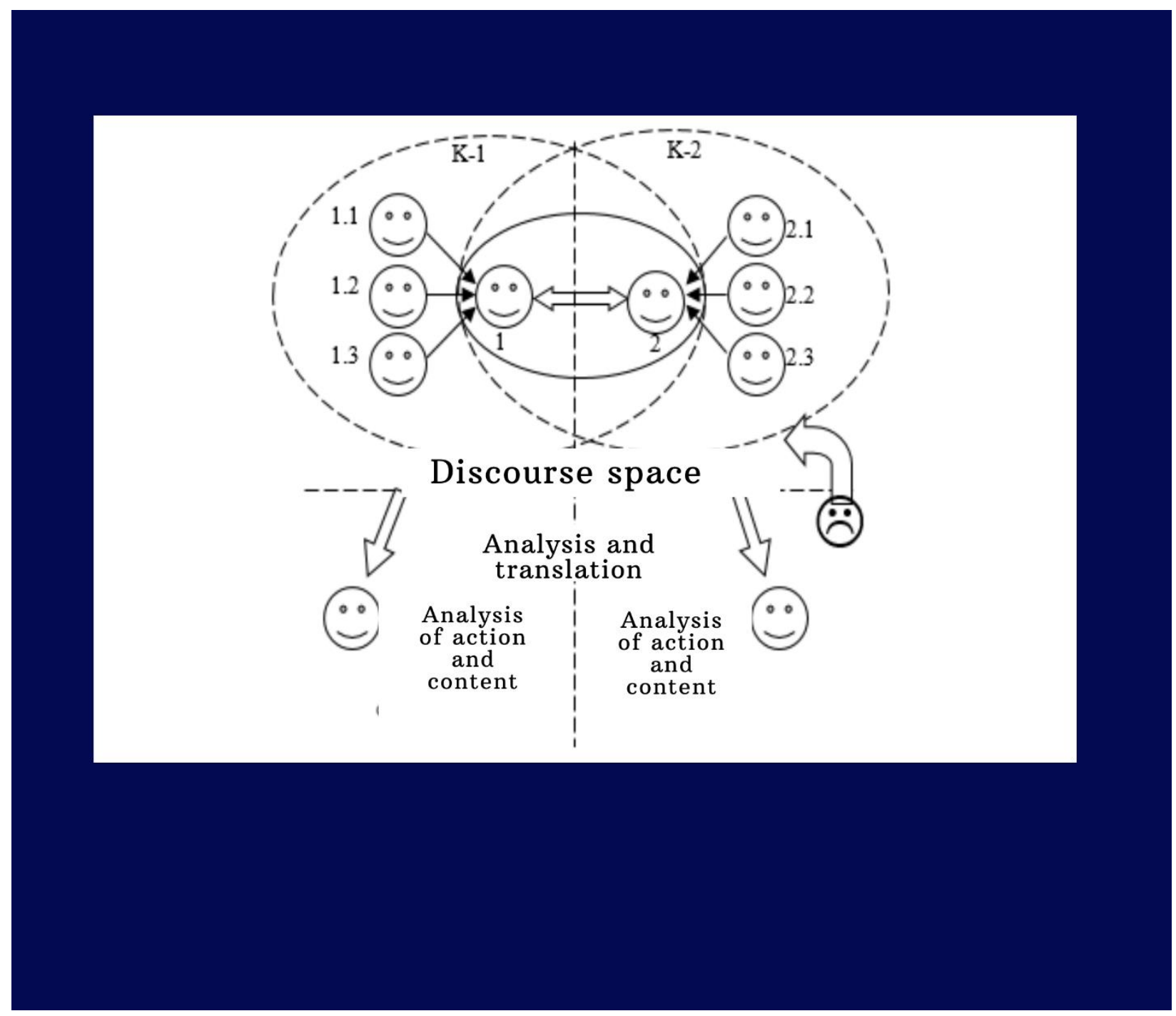

Fig. 16. "Discussion" game organization scheme

1. The discussion proceeds according to a given problem situation. Each team defends its own point of view. A prerequisite for a successful presentation is the structuredness and validity of the text which presuppose the presence of examples that illustrate the precedents of the solutions proposed by the team. The team consists of four members each of whom takes and maintains a certain position during the dispute. The theses that the team will defend are determined in advance.

1) Figures 1 and 2 are the main participants in the dispute, speakers representing the main theses. The position of the speaker can be occupied by postgraduates with any level of language training, since the text of the presentation is prepared at home. Speakers are constantly on the "arena of struggle". The main functions of figures 1 and 2 :

- presentation of the approving (for figure 2 - denying) team;

- presentation of the topic, justification of its relevance;

- definition of the terms used in the approval; 
- presentation of the point of view of the approving party;

- substantiation of aspects of consideration of this topic;

- presentation of arguments that will be proved during the game;

- transition to the proof of the arguments put forward; (Figure 2 refutes the arguments put forward);

- presenting a clear statement of the general line of approval.

The work of the denying team has its own characteristics which must be paid attention to postgraduates. First, the denying party must respond to all components of the affirmative thesis and respond to each argument. If an argument (or aspect) cannot be refuted more important arguments and comparisons are given.

When refuting the essence of the debate all components of the speech are considered: definitions, aspects, arguments, evidence, reasoning of the opponent, etc.

Each of the main figures (1 and 2) has a support group. The support group is out of the battlefield and is engaged in "understanding" what is happening in the arena. Nevertheless, at the same time, each figure from the support group can having understood what is happening, enter the arena, temporarily take the place of the main speaker and help him in the discussion, complementing, clarifying the argumentation.

2) Figures 1.1 and 2.1. - closely follow the course of the discussion focusing on finding weaknesses in the opponent's speech and argumentation. Their task includes:

- refutation of the denying thesis (new arguments cannot be given);

- posing cross-cutting questions.

3) Figures 1.2. and 2.2. substantiate and develop the main thesis of the team presented by the speaker, which involves the following actions:

- restoration of the team's point of view using hypotheses, examples, evidence;

- bringing new evidence;

- repetition of important aspects of previous evidence;

- detailed development of the team thesis;

- Bringing a clear formulation of the team's thesis;

- answer to cross-sectional questions.

4) Figures 1.3 and 2.3 highlight the moments when the dispute reaches a dead end, arguments are repeated or when a situation of misunderstanding arises. 
2. Organization of the space for translation and analysis (work begins after the end of the dispute).

There are two figures working in this space:

1) The figure of the content analyst - the task of the postgraduate (postgraduates) occupying this position includes the analysis of the discussion from the point of view of content. He should briefly describe what the essence of the problem under discussion was, what substantive justifications of the theses had one and the other team. The speech of the master student holding this position is, in fact, a summary.

2) The figure of an action analyst - the task of a master's student speaking in this position is to analyze the discussion from the point of view of the participants' actions.

He should describe who and what did during the dispute (for example: attack, defense, retreat, ridicule, resentment), and how the process of dispute unfolded as a result of these actions. In addition, this figure records a violation of the rules on which the debate should take place. The purpose of highlighting this position as a separate element of analysis is to focus on the features of behavior during a dispute and on compliance with the formal rules of communication. Special attention is paid to fixing possible errors in reasoning analyzing an action. The list of errors is in front of the postgraduate:

- question instead of an answer;

- walking in a circle;

- confusing statistics;

- cause and effect are confused;

- conclusions were made incorrectly;

- poor generalization;

- bias;

- attack not a position, but a person;

- argumentation like "it happens always and everywhere."

These two figures are external observers of the dispute and do not take part directly in it. Nevertheless, they can ask comprehension questions like:

"Did I understand correctly that ...?"

"I don't understand ... Could you clarify (repeat)?"

\section{Organization.}

The whole game is regulated by the figure of the organizer who monitors the sequence of theses and questions and ensures that the figures comply with their functions. The teacher in the subsequent performs the function of the organizer in the first lessons - the most "advanced" postgraduates. 
What is evaluated during the game?

1) Language competence:

- level of proficiency in linguistic and grammatical units worked out in the thematic series;

- no language errors;

- speech skills.

2) Intellectual competence:

- ability for critical thinking;

- cultural level (the ability to attract knowledge from different sources during a dispute).

3) Professional communicative competence:

- ability to evaluate (and maintain) one's own position, the position of the interlocutor;

- ability to fix the position of the opponent (includes separation, establishing a connection with the subject and purposes of the conversation, its sufficiency and credibility, reliability);

- ability to formulate and substantiate their own position;

- ability to oppose (questions, additions, elements of polemics, influence on the argumentation of the addressee of the conversation, that is, the addition of the interlocutor or objection to him);

- ability to reach a general conclusion.

4) Teamwork Ability:

- ability to assess their capabilities and take an optimal place in collective work (in the event that the participants in the dispute are not appointed by the teacher);

- ability to perform the function assumed (a sense of duty and responsibility).

The organization of a formal game according to the presented scheme allows you to directly work out and improve communicative professional skills as evidenced by the list of parameters that are subject to control.

It is not so much the result presented in the general conclusion that is important as the process of the dispute itself conducting a formal game, the observance of the forms of organizing the game since productive activity is stimulated professional communication skills are improved.

The main characteristic of formal debate is the existence of rules according to which opposing points of view clash. The organization of the debate according to certain rules allows you to keep the topic of discussion, does not allow you to turn the discussion into an argument when the opponent is attacked, and not considering the arguments. 
The productivity of the systematic inclusion of this type of work in the curriculum of the specialty language course was proved in the course of the experiment. A survey of postgraduates conducted at the end of the experiment shows that postgraduates rate the formal game «Discussion» most highly they note that during the course they acquire the skill of reflection which allows them to achieve better results when studying other disciplines.

Self-preparation of postgraduates for a discussion involves repeating all the material in a thematic series the need to rethink the content of familiar texts taking into account the request of a specific situation, a deeper penetration into linguistic and subject information. The effectiveness of this type of work is also carried out due to the need to constantly compare and analyze the familiar (practiced in the classroom) and new (independently obtained in other sources) information. In addition, differences in the legal norms of different countries allow a more meaningful approach to understanding the norms of Kazakhstani Law to see and rethink the nuances that become obvious thanks to specially organized forms of work.

The process of preparing for classes (independent work of postgraduates) is built on the basis of cognitive motives. The learners are distracted from the already familiar texts and enter the circle of new questions, which are a powerful stimulus for the formation of search and cognitive activity.

Thus, in the classroom carried out within the framework of problem-based learning to solve the basic objective of the course defined in the framework of a multidisciplinary approach: development and improvement of professional communicative abilities. It has already been repeatedly noted in this monograph that this course in the language of the specialty is designed for postgraduate students with a sufficient level of language training which is determined during the entrance control before the start of the course. This circumstance explains a certain methodological courage in setting the course objectives.

The fact that the course is elective and can be ignored by postgraduates forces them to look for new, more effective ways of working that attract the postgraduate not with the spectacular forms of work or the entertainment of the content of the course, but because they are based on cognitive motives.

All the efforts of the teacher are directed at satisfying the cognitive needs of the students in the independent solution of problem situations, in a higher level of independent creative activity.

Thus the teacher solves an important task: to develop and improve the linguistic and communicative competence of postgraduates at the postgraduate stage in the target language. Work on communication and speech skills and abilities are included in the process of forming and improving professional communication 
skills and constantly monitored by the teacher. In the description of the forms of work we have already noted that an indispensable criterion for the successful completion of an assignment for any of the types of work within the framework of problem-based learning is a systematic control of language and speech competence. The main methods of working with text were identified as follows: a) determination of the goals of reading, the choice of search orientation; b) isolation of semantic information blocks; c) identifying sources of information, d) fixing the extracted information; drawing up a short or short summary. These techniques correspond to such stages of working with the text as defining the interests of the positions behind the authors; understanding the purposes of the document; structuring information by the circle of persons referred to in the document.

After a circle of subject concepts has been established on the basis of which it is recommended to build a practical lesson in English at the postgraduate stage in the educational process the task of selecting texts for training is brought to the fore. Since according to the data obtained the dominant type of speech activity in the conditions of the native culture is professionally oriented reading it seems legitimate when selecting text materials to focus on the texts of formal publications serving it. We emphasize that in our opinion it is advisable to select text materials for teaching English from authentic English-language publications of the corresponding profile. The texts used in the English language-training manual for lawyers are taken from the materials of legal literature, both foreign and Kazakhstan and English speaking countries. Material (print, audio, video, visual) is considered to be authentic, which is a piece of real communication, i.e. created by a real author, a native speaker of the target language for a real audience, and not specifically for educational purposes (Voronina, 1994; Safonova, 1996; Hutchinson \& Waters, 1987; Robinson, 1991). Only in this case the use of a foreign language for communication purposes has real value and can be considered motivated and it is also possible to achieve the main goal of learning from the standpoint of intercultural communication. The achieving mutual understanding based on assigned professionally significant knowledge, correlated with knowledge about the world of a foreign language representative of the profession. Moreover, the university often as the last link in teaching a foreign language must provide an opportunity for students to get acquainted and work with real authentic sources of information.

It is important to formulate clear criteria that should guide the selection of texts. Since the teaching of a foreign language is interpreted as an organic part of the professional socialization of postgraduates adjuncts and applicants focused exclusively on the subject level of text organization. In this regard, speech and language characteristics should be considered at the final stage of selection as 
additional factors that most likely affect the processing of texts selected for training, the formation of an educational vocabulary, etc. (Uteubayeva, 2012).

Conformity of the subject characteristics of the source to the level of subject competence of learners. This criterion takes into account the basic value of the level of subject competence of postgraduates in the formation of content for teaching English. It is known that the postgraduate has certain information, knowledge about the described phenomenon, process, and things necessary to understand the offered professionally significant information, as a result of studying professionally oriented disciplines or scientific themes of postgraduates. It is especially important to consider this criterion when teaching since the subject matter of the texts should be selected because of studying the scientific research of postgraduates. It should be remembered that content that emphasizes incompetence weakens intrinsic motivation (Iskandarova, 1997), and hence the effectiveness of learning while meaningful content, other things being equal is better remembered (Rubinstein,1989). This criterion also involves taking into account the evolution of the subject competence of postgraduates which leads to a gradual complication of the subject content of education in general.

Repeatability and variability of subject content. The criterion for the variability of the subject content is due to the need to avoid a simple duplication of the content of professionally oriented disciplines. It assumes that when choosing a source of professionally significant information, preference will be given to the one that contains the subject characteristics familiar to the postgraduates at the time of reporting the information reported in the classroom in special disciplines. In other words, a method of informing is in demand in the process of teaching a foreign language in which new information is communicated about the elements of professional and industrial culture known to postgraduates. On the other hand, this criterion also assumes the repetition and variability of the subject content within the framework of the course itself.

Given these considerations it was decided to resort to the tactics of the dosed introduction of meaningful material, based on which it is possible to ensure a gradual saturation of educational communication with language primarily terminological material and a gradual increase in the volume of educational texts.

When highlighting topics, the emphasis was placed on the criteria proposed by Yu. A. Kudryashov, P.B. Gurvich (1982). These include:

1. Focus on the constant extraction and appropriation of new information. Information has the property of variable repeatability, each time it is supplemented by some new, previously unreported element of professional reality;

2. The capacity of theme that is, the predisposition of a theme to include elements of another theme as well as the possibility of using speech and linguistic 
(including professionally significant lexical) elements of a given theme in subsequent ones with a relative homogeneity of content;

3. The degree of thoroughness of the interpretation of the theme and in connection with this its division into smaller units;

4. The degree of richness of the theme with terminological vocabulary suggests that associative links should be strong: new terms should be thematically related; non-terminological words should be linked to the context of terminological vocabulary; vocabulary can be divided into smaller "portions" in accordance with the meaningful division of the theme;

5. The theme's predisposition to the comparison of key facts within the framework of the "dialogue of two cultures".

As a result, the following themes were included in the training content:

1) Justice and law in the RK, Russia, UK, USA.

a) Criminal proceedings;

b) Police Service;

c) Wanted. The pursuit. Investigative actions;

d) Criminal courts. Trial;

e) The system of punishment for crimes in the Republic of Kazakhstan, Russia, Great Britain and the USA;

f) Authorities administering punishment;

2) Civil proceedings;

a) Courts for civil cases in the Republic of Kazakhstan, Russia, Great Britain, USA;

b) Administration of justice;

c) Legal professions;

3) International aspects of modern law;

a) Activities of international organizations;

b) Democracy;

c) Human rights;

d) Conflicts and settlement agreements;

e) Drug business;

f) International terrorism.

For the disclosure of certain topics and their optimal assimilation by postgraduates, a set of different types of texts was created, including:

1. Verbal graphic texts:

a) introductory texts representing the main content information which 
constituting the core of the theme is gradually enlarged and detailed in the process of further assimilation of the theme by postgraduates;

b) microtexts - texts of short informational articles that provide development, concretization and illustration of thematic information which is presented in the introductory texts. Their thematic correlation with introductory texts determines the functioning of a certain range of terminological units with a situational variation of their use which contributes to their assimilation and the development of strong and flexible skills of abstracting and interpretation;

2. Texts - a part of the macrotext, providing a transition from the perception of monothemic to polythematic texts by increasing the volume and complication of their thematic content by combining two or three themes typical for macrotexts;

3. Macrotexts which are the most difficult for postgraduates in terms of volume thematic and terminological diversity. Working with them should complete the study of a specific theme and serve the purpose of strengthening and improving the skills of professionally oriented communication;

4. Verbal sign texts:

a) tables, maps and diagrams that visualize and supplement the information extracted by postgraduates from different types of verbal-graphic texts;

b) maps of conceptual fields which are independent communicative units that systematize and clarify the professional terminological base of the topic being studied.

The totality of educational texts is a system and each text in it has a professional, pragmatic, communicative and sociocultural value.

The professional value of the texts is due to the fact that all of them serve the activities of the international professional community, being part of its communication and information system satisfy the professional interests and needs of specialists providing relevant information in the specialty. From this point of view, they are focused on the future scientific and professional activities of postgraduates and serve as a standard of speech works that they may encounter in their future research activities.

The pragmatic value of the texts used is determined by the fact that postgraduates as additional in further research activities can use the information extracted from the study of the latter.

The communicative value of a set of authentic texts is determined by their orientation towards a specific sphere of communication within the framework of jurisprudence, as well as by the presence of communicative tasks aimed at assimilating the information with which the texts are saturated which activates the 
initiative of postgraduates to extract professionally and pragmatically significant data.

The socio-cultural value lies in the replenishment and deepening of the knowledge of postgraduates about the professional and industrial culture of the countries of the target language.

The foregoing has determined the choice of forms of work in the classroom. Classroom lessons on teaching professional-oriented communication were built according to the degree of increasing complexity. At the first stage, problematic tasks were chosen as the main type of work at the second stage the method of specific situations which involves improving the techniques of analysis, comparison, argumentation, etc.; at the third stage, a formal game was used. The professional and business sphere of communication, singled out as an educational base unit, determined the choice of certain linguistic and communicative means. Traditionally, linguistic means include a set of lexical and grammatical units that reflect a certain functional style, units of speech etiquette; to communicative - a set of speech actions, communicative tasks and situations that are implemented within the framework of this sphere of communication.

The definition of the goals of classroom and extracurricular activities and the choice of communication tasks were determined depending on the stage of work on a specific thematic series in such a way as to ensure a logically justified increase in the complexity of the organization of managed professionally-oriented communication.

Professional-oriented communication of lawyers follows its own rules. By definition it does not coincide with the features of natural communication which unfolds as a sequence of cycles, each of which is a conjugate act of communication partners. In natural communication the sequence of cycles is not predetermined by any the formed program, outlined plan. It is very labile and is directed by the very course of the joint activities performed. An important aspect of the communication process is the coordination of actions performed by its participants in time (The Interpretation Act, 1979).

As a result, we can provide the list of the communicative competences according to the model of postgraduate of law enforcement activity:

To argue the reasons that slow down the process of formation of the rule of law in Kazakhstan. Explain through the analysis of the main criminal law measures the implementation of criminal policy in this area, ways to solve problems, as well as improving national criminal, criminal procedure legislation, theory of evidence, criminology. 
To determine the problems associated with the application of the theory of evidence in the formation of the concepts of criminal procedure and national criminal procedure legislation.

Contextualize the National anti-corruption strategy in its relationship with the development of International law as a single integration process.

To determine the actual problems of criminological prevention the system of measures and methods of prevention of criminal offenses. To reveal the main problem aspects related to the victimological security of the individual.

Interpret the main categories the main institutions of the penitentiary system of the Republic of Kazakhstan. To regulate the risks of criminal activity as well as the prevention the role of administrative measures in ensuring the security of the state and people is high. Identify the main criminal risks in the field of economic (business and other) activities.

Analyze non-standard situations of law enforcement practice and develop various solutions to improve the theory and practice of criminal procedure legislation. To practice the basic theoretical approaches to innovations in conducting covert investigative actions.

To conduct a comparative analysis of the activities of law enforcement agencies of Kazakhstan and CIS countries, Europe, America and other foreign countries.

To formulate the reasons for the problems of law enforcement of justice and pre-trial investigation. To evaluate the features of the organization of the investigation, planning of the process the typical investigative versions, actions of investigator in typical investigatory situations.

Analyze the problems of systematization of information in the investigation of crime and decision-making for tactical impact on the investigative situation in the investigation.

Use the correct understanding of the role and purpose of penal policy in the aspect of combating crime. Compare public relations arising in connection with the Commission of crimes in the financial sphere using information technology. To determine the nature and distinctive features of the investigative action and operational investigative measures.

Formulate the author's concept of victimological security of the individual with the study and analysis of the main problems arising in this area. Use the basics of forensic technologies in the investigation of crimes by the internal Affairs bodies.

Simulate the production of investigative actions and operational investigative measures. Explain the reasons for the development of the penal system in Kazakhstan to solve the problems of execution of criminal punishment. 
Analyze qualified legal opinions and consultations on issues related to the activities of law enforcement agencies in the field of combating corruption, the economy. Use the basic methods and recommendations for the detection and investigation of crimes. Use the existing legal and organizational mechanism to ensure information security.

To recommend conceptual ideas and concrete proposals for the improvement of legislation and law enforcement practice in the field of combating organized crime and corruption; to formulate conclusions, proposals and recommendations to address the pressing problems of the organization of law enforcement. Predict the theoretical and practical problems associated with the organization of the investigation of a particular type of crime.

Develop recommendations to improve the activities of investigators at the initial and subsequent stages of the investigation of different categories of crimes. To find administrative and legal means and bases of ensuring public safety. Make a decision on a controversial scientific issue and find ways to resolve problematic situations in the criminal law sphere.

To propose concrete proposals on improvement of the current legislation regulating judicial and expert activity in the Republic of Kazakhstan, departmental normative legal acts and creation of a unified system of state expert institutions. To evaluate reasonable proposals to improve the activities of institutions and bodies executing criminal penalties in the Republic of Kazakhstan. To organize scientific research using the provisions of the rule of law, to be able to fruitful research and teaching activities.

To present information technologies in the financial and legal sphere, in the state sphere, in the civil sphere, in the criminal law sphere and in other spheres. Present the results of the work done in the form of reports, articles, designed in accordance with the existing requirements with the involvement of modern editing and printing. Propose management decisions related to the activities of law enforcement agencies in criminological planning and forecasting. Convince opponents of the most likely areas of scientific thought in the field of law enforcement.

It is obvious that judicial communication (in the broadest sense of the word) is a complex multifaceted process, conditioned by many factors and having a complex structure. This circumstance forces us to admit the impossibility of complete and accurate recreation of the selected type of communication in classroom lessons in the studied language. In this research an attempt was made to maximize the use of the basic components of judicial communication. We did not pursue the goal of imitating real-life processes bringing learning situations closer to the natural conditions of communication. On the contrary, the course on the 
development and improvement of professional communication skills and abilities was based on the types of work. The form of organization of which presupposes a reflexive attitude to one's own activity focuses not on the form of the statement but on its pragmatic orientation (which I do when I say X) which allows the transfer of professional communication skills and abilities to other areas of activity of postgraduates. In addition, classroom lessons built with an orientation towards speech professionally oriented communication are a condition for the formation of the psychological properties of a person, consciousness and self-awareness (Lomov, 1981).

The main goal of communication is the impact on the interlocutor achieved through careful planning of speech. So, the accusatory speech is built taking into account such elements as:

- $\quad$ socio-political characteristics of the crime, an indication of the characteristic features of the case, a statement of the program of the speech;

- $\quad$ statement of the factual circumstances of the case (plot of the case);

- $\quad$ analysis and assessment of the evidence collected in the case;

- $\quad$ substantiation of the legal qualification of the act;

- $\quad$ characteristics of the personality of the defendant;

- analysis and assessment of aggravating and mitigating circumstances;

- analysis of the conditions conducive to the commission of a crime and the formulation of proposals for their elimination;

- justification of the proposal for punishment;

- conclusion.

The defensive speech facing such elements as:

- $\quad$ socio-political assessment of the significance of the case, an indication of the specific features of the case, the introduction of general prerequisites for substantiating the position chosen by the defense, a reference to certain provisions put forward in the speech of the prosecutor;

- $\quad$ analysis of the factual and legal circumstances of the case;

- moral and psychological characteristics of the personality of the defendant and the motives of his behavior;

- final part.

It should be added that at present in the Kazakhstani court "competitiveness" is excluded in the speech of the prosecutor and the lawyer which increases the importance of intellectual work on the text. 
Another type of professionally prepared speech activity in an official situation is represented by such investigative actions as interrogation and confrontation (with the accused or suspect, witness, victim). Interrogation during the investigation of a crime is an investigative action. The essence of which is the receipt by the investigator from the interrogated information about the crime being investigated. Its circumstances and participants and on other issues related to the case, with the entry of this information into the protocol with strict observance of all established by the criminal procedure law rules.

For the investigator, interrogation turns out to be a prepared speech activity in a certain way. Since is a pre-predicted and planned formalized communication between the investigator and the interrogated during which a legally regulated effect on the mental, emotional, volitional spheres of the latter is carried out in order to obtain from him complete and correct information about the investigators' interests and the events, facts, relations known to him (Dulov, 1975).

The speech of the investigator in the process of preparing for interrogation or confrontation, although it is thought over in advance, is not written in advance (the protocol is written during the interrogation). His distinctive speech (as opposed to writing) is characterized by the presence of a specific addressee of the message and the possibility of influencing through feedback channels; high expressiveness and emotional richness of the speech message, the irreversibility of the speech message in time; the possibility of complex use of various sign systems - linguistic (language), paralinguistic (intonation), kinetic (facial expressions and gestures) (Komarkov,1975).

Another feature of judicial communication is its polylogic in content and monologue in form. O.E. Berkalova (1989) notes that in civil litigation the concept of an audience can be subdivided into several types of "interlocutors": the court, opposing parties, their representatives (lawyers); witnesses, prosecutor, expert, persons present in the courtroom. The statement of each with the exception of the latter presupposes an answer or a question from other participants in the process. In civil proceedings we are faced with dialogue both in the broad sense of the word understanding everything that was said during the consideration of a civil dispute and with the concept of dialogue in the narrow sense In this study we consider "dialogue in the broadest sense of the word" as a polylogue.

As the researchers note the judicial polylogue has its own characteristics. So, non-judicial communication is characterized by the fact that in the process of verbal communication there is an exchange of information, thoughts, judgments, mutual questions and answers. Moreover, all participants are in an equal position. The judgments of one side can be analyzed and criticized by the other side. The most 
typical example of such a dialogue can be considered a scientific debate, seminar, etc.

For any of the subspecies of judicial communication the peculiarity is that the addressee of information, person for whom the communication is carried out becomes not its participants but a third party, the court. Even when questions to the parties come from the court, and it seems to become an equal participant in the dialogue, there is no "pure" dialogue because no one can ask the court questions; express their opinion during the trial, the court is deprived of the right. $\mathrm{He}$ as if rising above the dialogue of the parties only directs it with his questions in order to briefly become a full participant in the dialogue at the end of the trial to make a judgment and announce it and thus give a final answer to all participants in the judicial dialogue in this process (Slesareva, 1980).

Thus, the sphere of professional communication of lawyers regulates the verbal and non-verbal behavior of communicants in the process of communication. Training in professional communication skills involves the allocation of a separate task of teaching prepared oral speech compiled in accordance with a given structure.

This circumstance determined the choice of types of work dominating in classroom lessons in the language of the specialty. The preference was given to works that allow developing and improving the following skills and abilities: analyze, compare, argue their own opinion, refute the arguments of the opponent, work in a team, and maintain a position (Uteubayeva, 2012).

Thus the organization of subject and speech actions in the classroom becomes the basis for teaching as the management of the postgraduate's mental activity since those subject and speech actions from which thinking is born and which direct it, in principle, are subject to control (Itelson, 1970). Achieving this goal is possible with the help of an appropriate setting of tasks; adequate forms of control as well as making the analytical-synthetic part to the external plan. The specifics of professionally oriented communication can be reflected in the program of the language of the specialty when using a problematic approach to build the course program.

As noted in the first chapter modern requirements for education and for the qualities that a modern specialist should possess suggest a change in teaching methods at the postgraduate stage.

The question of methods is especially relevant in the situation of training postgraduates, when the emphasis is shifted from the traditional question of methodology "how to teach" to the question "what to teach".

Speaking about teaching a language within the framework of an interdisciplinary approach we say that the goal of learning is to master professional communication skills that is, to master the ability to purposefully communicate, 
adhere to clear formalized (canonized) rules that ensure the achievement of goals. As has been repeatedly emphasized in this research, professional communication skills in the legal profession are both a tool and an essence of activity: the ways of implementing communicative intentions in professional communication are limited.

In the first chapter of the monograph, it was shown that when building a program in the language of a specialty at a law faculty it makes sense to focus on two basic communication skills: the ability to extract the necessary information, and ability to interpret (the limited number of hours devoted to language learning forces the methodologist to strictly observe the principle "Necessity and sufficiency"). The following reading criteria were identified as the content of the highlighted skills:

- determination of the goals of reading, the choice of search orientation;

- isolation of semantic information blocks;

- identification of sources of information available in this dossier by their origin, legal value, meaning;

- fixation of the extracted information.

Communicative "speaking" refers to the techniques used in interpersonal communication and includes:

- establishing contact based on an assessment of one's own position, position of the interlocutor and meaning of the conversation;

- fixing the position of the addressee of the conversation (includes the inducement to transfer information, its separation, establishment of a connection with the subject and goals of the conversation, its sufficiency and credibility, reliability);

- formulation and justification of their own position;

- opposition (questions, additions, elements of polemics, influence on the argumentation of the addressee of the conversation, that is, the addition of the interlocutor or objection to him).

For this purpose in one of the first classes a questionnaire was conducted which aimed to determine the nature of motivation for learning and the scope of scientific interests of postgraduates, and testing, which due to the lack of experience of professional communication was also considered by us as a pre-experimental section.

The material for the test was textual excerpts with a general scientific orientation and focused on general erudition and acquired knowledge at the university by postgraduates. One hundred twelve postgraduates took part in the testing. As a result, it was found that the fundamental skill of referent professionally oriented reading, the ability to determine a topic is formed much worse than the 
ability to extract information from the source on which informative reading is based (Table 4 and Figure 17).

Table 4. Reading comprehension test results:

\begin{tabular}{|c|c|c|}
\hline \multirow[b]{2}{*}{$\begin{array}{l}\text { Number of tested } \\
\text { postgraduates }(\%)\end{array}$} & \multicolumn{2}{|c|}{ Skills } \\
\hline & Defining the theme & $\begin{array}{l}\text { Extracting the } \\
\text { information }\end{array}$ \\
\hline 100 & 33 & 67 \\
\hline
\end{tabular}

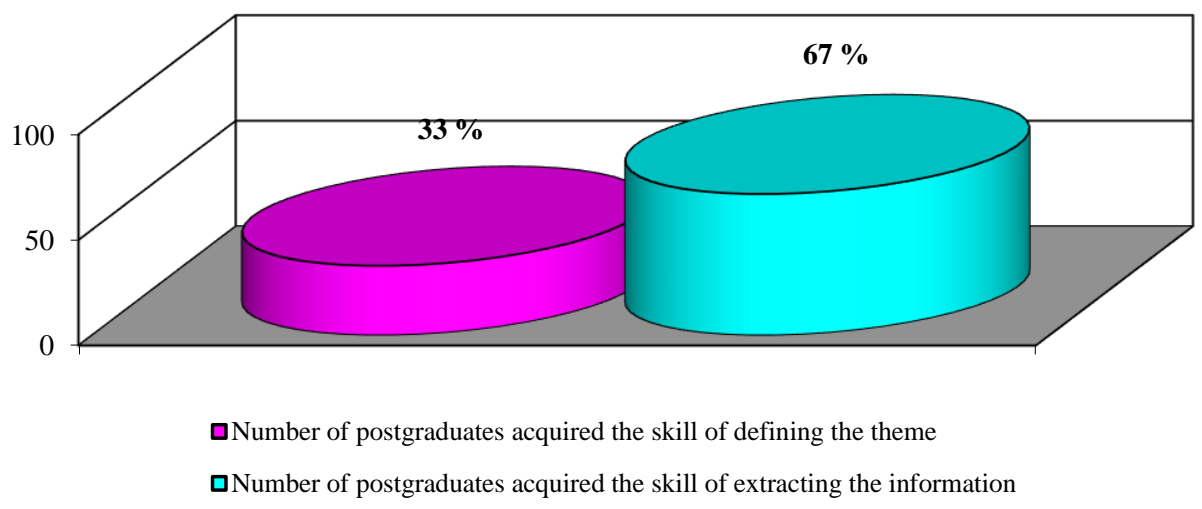

Fig. 17. Reading Comprehension Test Results

Nevertheless, there was a significant gap in the formation of the main skill, depending on the explicitness / implicitness of the topic and the presence of support in the form of the given choice (Table 5 and Figure 18).

Table 5. The achievement test results of the main skill of reference reading:

\begin{tabular}{|c|c|c|c|c|c|}
\hline \multirow{2}{*}{$\begin{array}{c}\text { Skill of } \\
\text { defining the } \\
\text { theme }\end{array}$} & \multirow{2}{*}{$\begin{array}{l}\text { Accordi } \\
\text { ng to the } \\
\text { title }\end{array}$} & \multicolumn{2}{|c|}{$\begin{array}{l}\text { Explicitly } \\
\text { expressed }\end{array}$} & \multicolumn{2}{|c|}{$\begin{array}{l}\text { Implicitly } \\
\text { expressed }\end{array}$} \\
\hline & & $\begin{array}{c}\text { in the } \\
\text { beginning } \\
\text { of passage }\end{array}$ & $\begin{array}{l}\text { at the end } \\
\text { of the } \\
\text { passage }\end{array}$ & $\begin{array}{l}\text { without } \\
\text { choice }\end{array}$ & $\begin{array}{l}\text { with } \\
\text { choice }\end{array}$ \\
\hline $\begin{array}{l}\text { Number of } \\
\text { tested } \\
\text { postgraduates } \\
\qquad \%)\end{array}$ & 11,55 & 51,3 & 2,85 & 6,1 & 28,2 \\
\hline
\end{tabular}


$\square$ according to the title

口explicitly expressed at the end of the passage

- Implicitly expressed with choice
Dexplicitly expressed in the beginning of the passage

口Implicitly expressed without choice

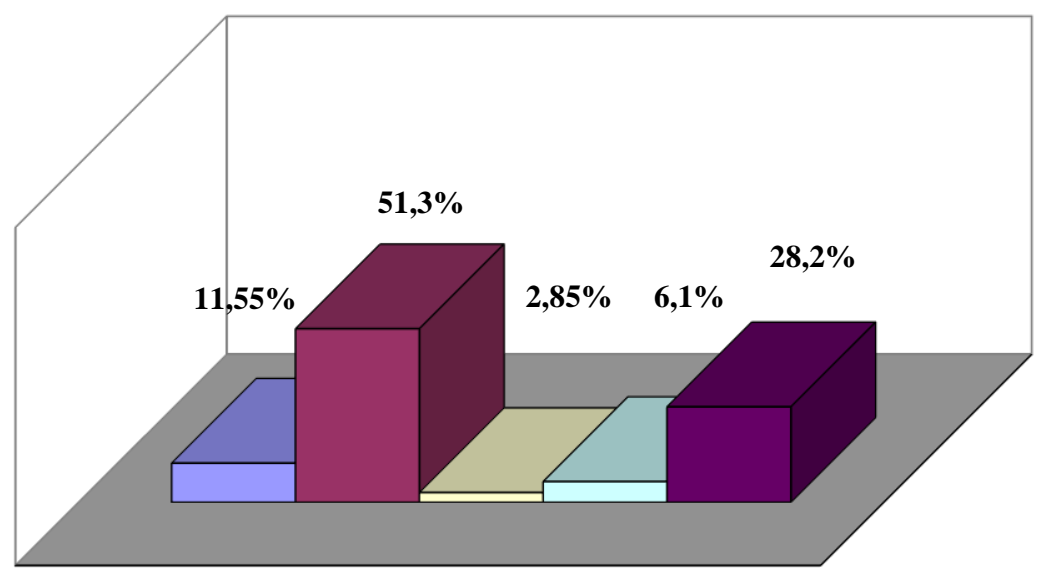

Number of postgraduates acquired the skill of defing the theme

Fig. 18. The achievement test results of the main skill of reference reading

The best indicator of understanding was given by the passage in which the explicit referent was located at the beginning of the passage. Almost equal difficulties in determining the theme of the read were caused by the location of the explicit thematic referent at the end of the text passage and the establishment of the implicit theme. However, an increase in the latter indicator by seven times in the presence of a choice of already formulated themes indicates not so much a lack of understanding of the topic but rather an inability to formulate it independently by almost a third of the postgraduates. In addition, significant difficulties arose in determining the topic and general content of the title as a result of which most of the test takers limited themselves to translating them.

In general, the test results indicate unequal and moreover very different levels of formation of skills depending on the way of expressing thoughts while the ability to navigate sources in a deductive way of expressing thoughts is developed much better than others. Thus, the texts of scientific articles best correspond to the nature of the former foreign language speech skills of postgraduates from the point of view of the logical and compositional structure which once again confirms their suitability for use in teaching a professionally oriented foreign language.

As a result, the organization of teaching a professionally - oriented foreign language based on the texts of authentic scientific articles allows the postgraduate to gain real experience of special communication at the postgraduate stage. 
Thus, the main directions of the methodology developed by us for the formation of foreign language communicative competence of lawyers in the system of postgraduate education are:

$>$ use of the communicative-activity method in the framework of problem-based learning;

$>$ combining two methodological tasks in the educational process (improving foreign language and professional competence);

creating in the process of teaching a professionally oriented foreign language an actual motivation for professional communication;

$>$ gradual formation of professional communication skills and abilities which implies a gradual increase in the complexity of problem tasks and the complication of pragma-professional tasks in a foreign language in accordance with the level learning model.

The effectiveness of the developed methodology was tested in the course of an experiment, the contingent of which included postgraduates of the 1st faculty of the Academy of the Ministry of Internal Affairs of the Republic of Kazakhstan (training of senior management personnel for the bodies of the Ministry of Internal Affairs of the Republic of Kazakhstan), adjuncts of the Academy of the Ministry of Internal Affairs of the Republic of Kazakhstan postgraduates and adjuncts of the Karaganda Law Institute of the Ministry of Internal Affairs of the Republic of Kazakhstan.

By a pedagogical experiment, modern pedagogy of higher education understands a research method that is used to clarify the effectiveness of the application of certain methods and means of teaching and upbringing.

An important place is given to pedagogical experiment, since in its orientation it is designed to solve at least two main tasks:

1) obtaining experimental data characterizing the pedagogical conditions for the formation of foreign language communicative competence of lawyers at the postgraduate stage of preparation and allowing the introduction of targeted methods for solving research problems;

2) testing hypotheses about the effectiveness of the methodology for the formation of foreign language communicative competence of lawyers.

The hypothesis of our research was that the effectiveness of the formation of foreign language communicative competence of postgraduate lawyers studying in the specialty "Law enforcement" increases if the learning process is carried out:

- based on the pan-European levels of communicative competence;

- taking into account the professional and communicative needs of learners; 
- on the basis of educational materials and methods, formed in accordance with the basic principles of pedagogical hermeneutics.

Based on the requirements of the State Educational Standard of Foreign Languages for specialists undergoing postgraduate education (master's students), the manuals and different resources aimed at developing postgraduates' skills of independent reading, interpretation and interpretation of the original scientific literature in the specialty, quick extraction of the necessary information within the legal subject, conducting scientific mono- and polylogue, annotation and abstracting of the authentic text of the legal profile. The structure and content of the aforementioned manuals were completely focused on achieving the set goal - the formation of a foreign language communicative competence of a lawyerresearcher.

The author strictly adhered to the principles of pedagogical hermeneutics defined above, which led to the structural uniformity of each lesson. The structure of the lesson, calculated on average for 3 practical sessions, includes:

- an overview of the lexical material of the current lesson;

- introductory and viewing reading of the microtext;

- special exercises for the formation of the skill of interlingual and intralingual transformation;

- comparative analysis of parallel microtexts in English and native language;

- introduction of clichés for the construction of original microtexts by master students on the topic of the lesson

- performing exercises for compiling microtext (essays);

- reading, abstracting macrotext;

- discussion of issues for discussion or a specific situation;

- drawing up a summary (annotations) based on the materials of the passed theme.

The skills of comprehension of the written text for further presentation of its main content and interpretation are developed with exercises that require simultaneous switching from written text to oral speech and from one language to another. The skills of cross-lingual and intra-lingual transformation of text are worked out by exercises in which you need to select synonyms, find descriptive phrases, highlight words with key information, find lexical units for pivotal points of memory and for records.

Active proficiency in a language implies the speaker's ability to express the same meaning in a variety of ways - synonymously, peripherally and idiomatically - observing the rules of lexical and grammatical compatibility. In most cases, these 
rules are of an intralingual nature - they operate only in a given language and therefore, when mastering a foreign language, they require memorization.

Postgraduates ideally perform exercises for writing essays or abstracting both micro- and macro-texts by introducing clichés. To achieve the goal of working with the means of scientific presentation, which are not separate and disparate classes of words, not connected to each other, but a single system of deeply and actively interconnected means of translating communicative intentions, postgraduates are offered schemes of annotations and resumes. So, a text from twenty to seventy pages was replaced by a summary of two to three pages, forming a microtext that includes a minimum number of terms, does not have repetitions ("noise") and is composed of key phrases and fragments characteristic of a given text containing several words of a general scientific nature and therefore relevant to the organization of the scientific text in principle. Postgraduates are offered schemes for writing resumes or abstracts, as a result of which scientific articles and monographs are processed, which are subsequently required to pass examinations in English in the amount of the candidate's minimum Level $\mathrm{C} 1$ / C2.

We offer one of the schemes:

- This book is a collection of articles on P / the diversity of kinds of information/ reflects the current knowledge in P/ educational uses of $P$.

- The author frames the central question of his monograph as follows: " $P$ "; in answer to the question, the author proposes $Q /$ pursues the question whether $P$ by breaking it down into two sub questions, $R$ and $Q$.

- Accordingly, the book/paper falls into two halves: $P$ and $Q$.

- The (methodological) (sub)questions discussed in Part 1 of this monograph, reads as follows: $P$.

- The bulk of this chapter is devoted to $P$.

- In F's approach $P$ is viewed as $Q$.

- $F$ develops an account which brings out a picture of $P$, treating a number of problems that have traditionally been recognized to be especially problematic areas for $Q$ theories, and then proceeds to investigate the nature of $R$ and sets out to provide a definition for $Q$.

- The author brings the following example into the discussion, $P$.

- The book has a variety of virtues; I do, however, have certain criticisms. One concerns $P$. Another criticism concerns F's discussion of $Q$. The most serios weakness of the book is that $P$. The author fails, in my view, to make a convincing (enough) argument for his proposed reduction of $P$.

- F's book is timely and important / is clearly written and the arguments convincing. Many possible research questions are raised. F's book has raised many intriguing problems and will be a stimulus for a great deal of productive theoretical 
and descriptive research on the phenomena. The book's style is engaging and light. $F$ 's book is very well done in every respect/ F's endeavor is successful.

- I recommend it highly. In brief, the theoretical value of the book is obvious.

- This is a useful introduction to the discipline / field / sphere, which can be recommended to anyone who wants a way in two the full range of definite problems.

Thus, these exercises contain in detail and systematically ordered expressions, phrases and examples characteristic of the English scientific style, allowing you to simply, clearly and clearly express your thoughts and link them into a single whole - a summary, abstract, thesis, report, review, etc. The exercises also model the linguistic competence of a native speaker, the speech of the author of a scientific presentation, features of the scientific style in English and the rules of idiomatic expression of meaning in the English language system.

In the performance of these exercises, a self-learning mechanism is incorporated, and not the one that imitates the traditional "school" teaching a foreign language, based on explanation and memorization, but one that naturally assimilates the native language, and which acts on the basis of analogy, reproducibility, selfdevelopment, inherent in the language itself and ensuring its automatic, subconscious assimilation.

Useful for the development of switching skills and the implementation of several actions at once is an exercise in which the trainees are invited to simultaneously familiarize themselves with authentic microtexts, located in parallel columns. Many exercises work out the technique of oral speech, since a master's student (lawyer) must have good diction, a high culture of oral speech, a significant supply of reproductive material in both native language and English. A lawyer has to work under the conditions of initial data, so a number of exercises are specifically focused on developing skills for working with incomplete information and developing language guesses (intuition).

To study certain themes and achieve their optimal assimilation in practical classes, verbal graphic and verbal-sign texts were used. Verbal graphic texts contribute to the assimilation and development of strong and flexible skills of abstracting and interpretation, while verbal-sign texts systematize and clarify the professional terminological base of the studied theme.

Illustrative material, i.e., diagrams, maps, statistical data, which are independent communicative units, served not only as examples, but also as one of the main sources of information, on which, in turn, the manual is based, therefore and processing has been given special attention. The importance of this section in the study of the topic, in our opinion, is extremely high, since video graphic materials act as a symbolic support in the production of a monologue (a summary 
on the topic under study) and dialogical speech (specific situations, discussions) by a master student.

During the experiment, a testing method was also used, the purpose of which was to identify the level of formation of a given feature using a pedagogical test.

Each lesson, which took place in the experimental groups, consisted of three stages: theoretical, practical and control.

At the practical stage, postgraduates were offered exercises that involve them in the communication process: discussions (dialogues) on a given topic, analysis of practical situations, role-playing games, as well as listening, during which the postgraduates were asked not only to repeat the text of the audio recording, but also to express their opinion about the set in the text of the recording of the problem or offer your point of view from the position of one of the participants in the recorded dialogue. Usually, discussion of discussion themes took place in groups of 2-3 people, then a collective opinion formed in a group or several individual opinions was presented to the entire audience of students, if the students could not come to a common opinion on the issue under discussion. Such discussions made it possible for students to develop the skills of both dialogical and monologue speech, acquire the ability to logically formulate their thoughts and defend their opinions. $t$ should be noted that postgraduates were always offered several scenarios of speech exercises, which made it possible not to repeat the same scenario several times in class. This ensured constant involvement in the learning process not only of the direct participants in the game, but also of the audience.

At the final, control, stage, the trainees were asked to complete either a written Writing task, for example, writing a plan, press release essay or article, or to answer in writing questions regarding their own solution to a particular problem posed within the text material.

When assessing the oral and written answers of trainees, we were guided by the following criteria for the formation of a foreign language communicative competence:

Reconstructive-variable:

1) the ability to listen to information;

2) the ability to adequately respond to questions;

3) skills of establishing contact with colleagues in a foreign language for organizing joint activities;

4) the ability to correctly present the listened or read information;

5) the ability to highlight the main idea of information when viewing the reading of scientific legal literature in a foreign language;

6) the skill of determining the meaning of a word based on knowledge of legal terminology; 


\section{Partial search:}

1) listen to scientific legal information, highlight and abstract;

2) be able to isolate the main information in the text;

3) the ability to highlight additional information in the message;

4) be able to clarify the main points of the listened (read);

5) be able to analyze the content of a scientific legal text according to diagrams, figures, statistical data;

6) be able to select sentences containing the main information;

7) the ability to highlight sentences containing additional information;

8) be able to isolate keywords from the text;

9) be able to extract information from a scientific legal article for compiling a message on a topic in a foreign language;

Transformational:

1) the ability to find confirmation of certain facts, events in a scientific and legal text;

2) the ability to find in a scientific and legal text a refutation of certain facts, events;

3) be able to provide a justification for the proposed title to a legal article;

4) be able to correct the proposed plan for the article;

5) be able to draw up a plan for the text in the form of affirmative or interrogative sentences;

6) be able to draw up abstracts for a scientific article;

7) be able to convey the content of a scientific article using reconstructed sentences;

8) be able to compose your own message on the research topic, using information from several scientific legal articles;

9) be able to conduct a discussion on the topic of the message.

It should be noted that when assessing the level of formation of the communicative competence of postgraduates, the self-assessment of these skills by the postgraduates themselves was also taken into account, which included the following indicators:

Professionally significant qualities:

1) development of skills of independent work;

2) the ability to work both individually and in a team;

3) possession of formulas of politeness; the ability to self-esteem; the ability to act in non-standard situations;

4) tolerance;

5) the ability to listen to others; behave politely, tactfully, diplomatically, restrained; 
6) the ability to build your report, abstracts, message to the conference;

7) the skill of collecting material on a scientific topic;

8) be able to argue.

Motives of educational activity:

1) become a highly qualified lawyer with knowledge of a foreign language;

2) knowledge of a foreign language gives access to new professional legal information;

3) the desire to work with foreigners without an interpreter or abroad after graduation from the magistracy;

4) I like to study a foreign language;

5) successfully pass a qualifying examination in a foreign language;

6) my future well-being will depend on the knowledge of a foreign language;

7) the desire to know a foreign language, just in case, will come in handy in life;

8) successfully study and improve professional level;

9) gain the approval of management and others.

Factors of academic success:

1) studying information about legal communication with colleagues;

2) retrieving information about new research, methods in jurisprudence;

3) the teacher's interested attitude to the knowledge of the master's student;

4) the teacher coordinates and advises the activities of postgraduates;

5) postgraduates' feeling of their own competence;

6) flexible forms of control of knowledge of postgraduates;

7) providing postgraduates with greater creative activity;

8) Master's students possessing compensatory skills when working with original scientific literature;

9) personal example and teacher's interest in the results of their work;

10) individualization and differentiation of training depending on the initial level of knowledge and interests of postgraduates;

11) purposeful study of original scientific materials related to the profession.

Formation (unformed) of communication skills:

1) the ability to read and write an annotation, review, thesis, define the main idea and convey it in the form of a dialogue or monologue;

2) establish contact with colleagues to organize joint activities - participation in the forum, preparation for the conference, lectures; 
3) establish contact with the person (accused, suspect) during the interrogation;

4) the ability to ask, listen; give recommendations;

5) the ability to prepare a report, message, speak at a conference, "operative", read a lecture;

6) understanding of the oral message of legal content at the conference and presentation of the listened information using scientific vocabulary.

Experimental teaching was chosen as a method of verification due to the fact that it does not disrupt the natural course of the educational process.

\section{Purpose of experimental learning.}

As already noted, the change in general requirements for the level of training of specialists at the postgraduate stage requires the introduction of new methods of work and the improvement of traditional ones.

The need to use motivation that is significant for a master's degree student in learning a language, taking into account the principle of individualization of training, and, most importantly, the need to co-organize work in an interdisciplinary space of requirements for the level of professional communicative competence of lawyers, determined the main goal of the experiment.

Based on the analysis of the methodological literature, as well as based on the identified shortcomings in the level of proficiency of graduates of the law faculty of postgraduate education in professional communication skills and abilities, we put forward the hypothesis that targeted training in professional communication skills and abilities should be included in the curriculum of the course in the language of the specialty.

\section{Training material}

Experiential training was carried out on the basis of a program we had drawn up. Each topic-specific experiential learning session corresponds to a program. The tasks were lined up as the complexity of the forms of work and the level of communication skills and abilities increased.

\section{The main stages of training}

1. Preparatory work and stating section.

2. Experimental learning.

3. Post-experimental section.

Next, we will consider in more detail the content of the above stages.

1. The preparatory work consisted of: 
- in observing the educational activities of postgraduates, adjuncts and law applicants;

- in studying the experience of colleagues;

- in the analysis of literature data on research problems.

This stage convincingly proved the need to search for new approaches to the process of teaching foreign language communication, in particular, professionally oriented communication, which consists of skills and abilities of working with a scientific text, extracting the necessary information, abstracting taking into account the specialty. During the preparatory stage, the factors both contributing and hindering the effectiveness of the process of teaching English at the postgraduate stage were carefully studied. These include the following:

1) factors contributing to the effectiveness of the learning process:

- standard degree of trainees' proficiency in special legal terminology;

- a high degree of motivation and activity;

- "maturity" of the mentality.

2) factors impeding the effectiveness of the learning process:

- insufficient number of teaching hours to achieve the level of free scientific proficiency in a foreign language and the processing of monographs and scientific materials necessary for research activities;

- different levels of English proficiency among postgraduates;

- lack of comfortable and comfortable classrooms equipped with modern technical teaching aids (satellite TV, multimedia classes);

- methodological unpreparedness of the majority of teachers to conduct foreign language classes in non-linguistic universities at the postgraduate stage.

The above factors were taken into account when developing a program for experimental learning.

The ascertaining section consisted of 2 parts. The first part was essentially a diagnostic test, which was offered to all students at the postgraduate stage who studied English at a university, with the aim of distributing them into groups of the appropriate level. At the same time, not only knowledge of vocabulary, grammar and fluency of speech (with oral answers) were taken into account, but also the "maturity of mentality", the willingness to think critically on their own, logical reasoning of their point of view.

1. A written test compiled according to the syllabus examination requirements for postgraduates studying in the specialty $6 \mathrm{~N} 0303$ "Law enforcement" in the universities of the Ministry of Internal Affairs of the Republic of Kazakhstan.

The written part (test) was checked by keys, which excluded the subjectivity of the assessment, however, due to the impossibility of ensuring the "purity" of the experiment, the number of postgraduates who received higher scores for the written 
test differs from the number of postgraduates with high scores for the oral answer in a comparative respect ... The score for the written answer was given taking into account the criteria.

2.Interview, during which the student was asked to reveal one of the problematic topics, for example:

- Habeas Corpus is part of a Latin phrase - Habeas Corpus ad subjiciendum that means "Let the body be brought before the judge".

- Why is the jury called "the bastion of liberty"?

- Society is not ready to accept ex-prisoners. They will always be objects of suspicion in the community.

- Thousands of people are imprisoned in inhumane conditions. Human rights.

- How did the police force as law enforcement organization arise and develop?

Based on the results of the first ascertaining section (Table 6 and Figure 19), two groups were identified that were maximally identical in composition (age) and in the level of English proficiency.

Age - from 23 to 40 years old. The quantitative composition of the experimental groups is 112 people, of which the experimental group is 56 postgraduates; control group - 56 postgraduates.

Table 6. The results of the first ascertaining test:

\begin{tabular}{|c|c|c|c|c|c|c|c|c|c|}
\hline & \multicolumn{3}{|c|}{ Test } & \multicolumn{4}{|c|}{ Spoken replies } \\
\hline & & & $\begin{array}{c}\text { Less than } \\
60 \% \\
\text { correct } \\
\text { answers }\end{array}$ & $\begin{array}{l}60-80 \% \\
\text { correct } \\
\text { answers }\end{array}$ & $\begin{array}{c}\text { More than } \\
80 \% \\
\text { correct } \\
\text { answers }\end{array}$ & $\begin{array}{c}\text { Excell } \\
\text { ent }\end{array}$ & Good & $\begin{array}{c}\text { Satisfac } \\
\text { tory }\end{array}$ & $\begin{array}{l}\text { Unsatisfa } \\
\text { ctory }\end{array}$ \\
\hline \multicolumn{2}{|c|}{$\begin{array}{c}\text { Total number of } \\
\text { postgraduates }\end{array}$} & 112 & $\begin{array}{c}31 / \\
27,6 \%\end{array}$ & $\begin{array}{c}68 / \\
60,7 \%\end{array}$ & $13 / 9,9 \%$ & $\begin{array}{c}9 / \\
11,6 \\
\%\end{array}$ & $\begin{array}{c}44 / 39,3 \\
\%\end{array}$ & $\begin{array}{l}49 / 43 \\
75 \%\end{array}$ & $\begin{array}{c}8 / 7,15 \\
\%\end{array}$ \\
\hline \multirow{2}{*}{$\begin{array}{l}\text { inclu } \\
\text { ding }\end{array}$} & $\begin{array}{c}\text { Number of } \\
\text { postgraduates } \\
\text { in } \\
\text { experimental } \\
\text { group } \\
\end{array}$ & 56 & $\begin{array}{c}13 / 23,2 \\
\%\end{array}$ & $\begin{array}{c}35 / \\
62,5 \%\end{array}$ & $\begin{array}{c}8 / 14,3 \\
\%\end{array}$ & $\begin{array}{c}7 / \\
12,5 \\
\%\end{array}$ & $\begin{array}{c}25 / 44,6 \\
\%\end{array}$ & $\begin{array}{c}22 / \\
39,3 \%\end{array}$ & $2 / 3,6 \%$ \\
\hline & $\begin{array}{c}\text { Number of } \\
\text { postgraduates } \\
\text { in control } \\
\text { group }\end{array}$ & 56 & $\begin{array}{c}18 / 32,1 \\
\%\end{array}$ & $\begin{array}{c}33 / \\
58.9 \%\end{array}$ & $5 / 9.0 \%$ & $\begin{array}{c}4 / 7,1 \\
\%\end{array}$ & $\begin{array}{c}19 / 34.0 \\
\%\end{array}$ & $\begin{array}{c}27 / \\
48,2 \%\end{array}$ & $\begin{array}{c}6 / 10,7 \\
\%\end{array}$ \\
\hline
\end{tabular}




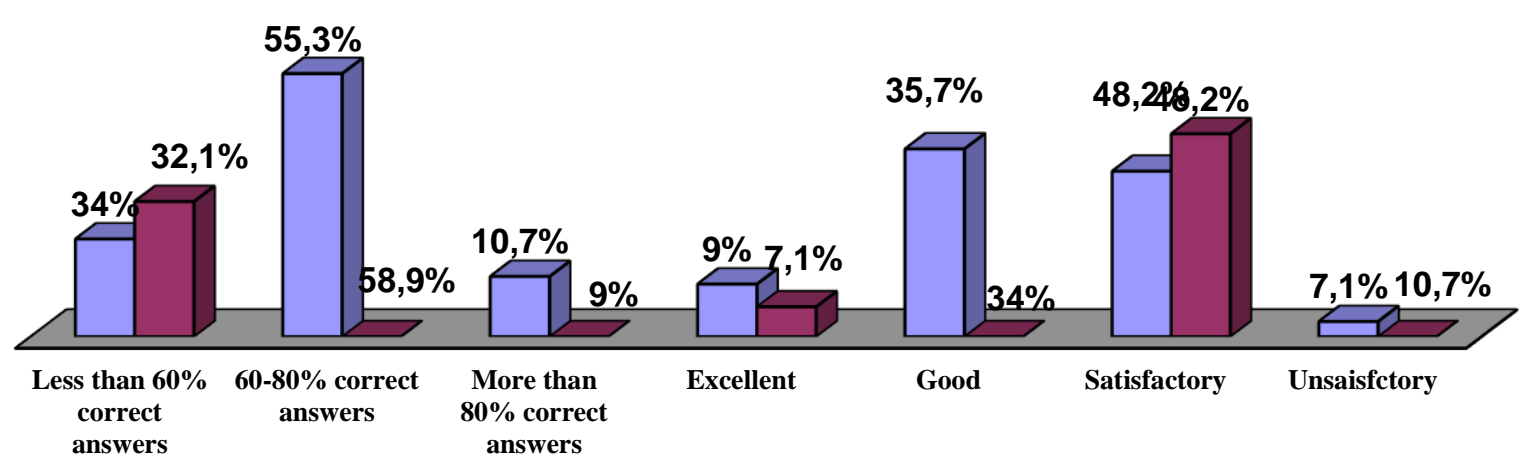

Figure 19. The results of the first ascertaining test

The second part of the ascertaining section was carried out in the experimental and control groups and consisted in writing a short essay (200-250 words) containing a problematic question to choose from. The content of the work made it possible to determine the level of proficiency not only in writing skills, but also to find out the skills of presenting and abstracting text. In general, the ascertaining section made it possible to establish the initial level of master's students' mastery of oral (monologic and dialogical) and written speech within the framework of university requirements, as well as to reveal the presence or absence of their previously formed skills of professional foreign language communicative competence. The results of the second ascertaining section for the control and experimental groups are presented in Table 7 and Figure 20.

Table 7. The results of the second ascertaining test:

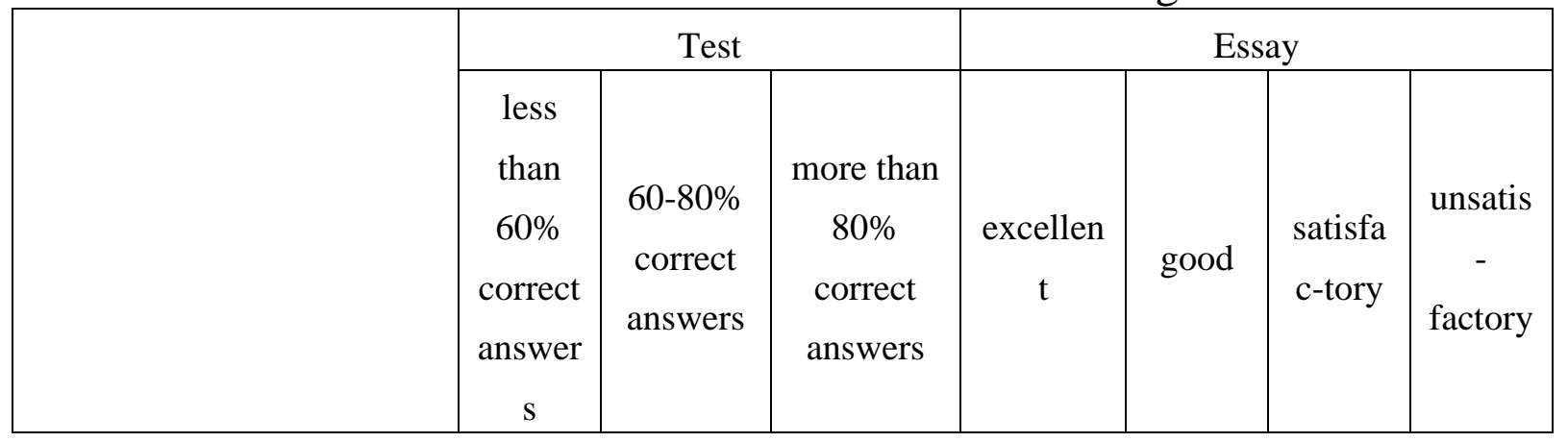




\begin{tabular}{|c|c|c|c|c|c|c|c|c|c|}
\hline \multicolumn{2}{|c|}{$\begin{array}{c}\text { Total number of } \\
\text { postgraduates }\end{array}$} & 112 & $\begin{array}{c}25 / \\
22,3 \\
\%\end{array}$ & $\begin{array}{c}70 / 62,5 \\
\%\end{array}$ & $\begin{array}{c}17 / 15,2 \\
\%\end{array}$ & $4 / 3,5 \%$ & $\begin{array}{c}38 / \\
33,9 \%\end{array}$ & $\begin{array}{c}45 / \\
40,2 \\
\%\end{array}$ & $\begin{array}{c}25 / \\
22,3 \%\end{array}$ \\
\hline \multirow{2}{*}{$\begin{array}{l}\text { incl } \\
\text { udi } \\
\text { ng }\end{array}$} & $\begin{array}{c}\text { Number of } \\
\text { postgraduates } \\
\text { in experimental } \\
\text { group }\end{array}$ & 56 & $\begin{array}{c}7 / \\
12,5 \%\end{array}$ & $\begin{array}{c}38 / \\
67,9 \%\end{array}$ & $\begin{array}{c}11 / \\
19,6 \%\end{array}$ & $4 / 7,1 \%-$ & $\begin{array}{c}26 / \\
46,4 \%\end{array}$ & $\begin{array}{c}18 / \\
32,1 \%\end{array}$ & $\begin{array}{c}8 / \\
14,3 \%\end{array}$ \\
\hline & $\begin{array}{c}\text { Number of } \\
\text { postgraduates } \\
\text { in control } \\
\text { group }\end{array}$ & 56 & $\begin{array}{c}18 / \\
32,1 \%\end{array}$ & $\begin{array}{c}32 / 57,2 \\
\%\end{array}$ & $6 / 10,7 \%$ & - & $\begin{array}{c}12 / \\
21,4 \%\end{array}$ & $\begin{array}{c}27 / \\
48,2 \%\end{array}$ & $\begin{array}{c}17 / \\
30,4 \%\end{array}$ \\
\hline
\end{tabular}

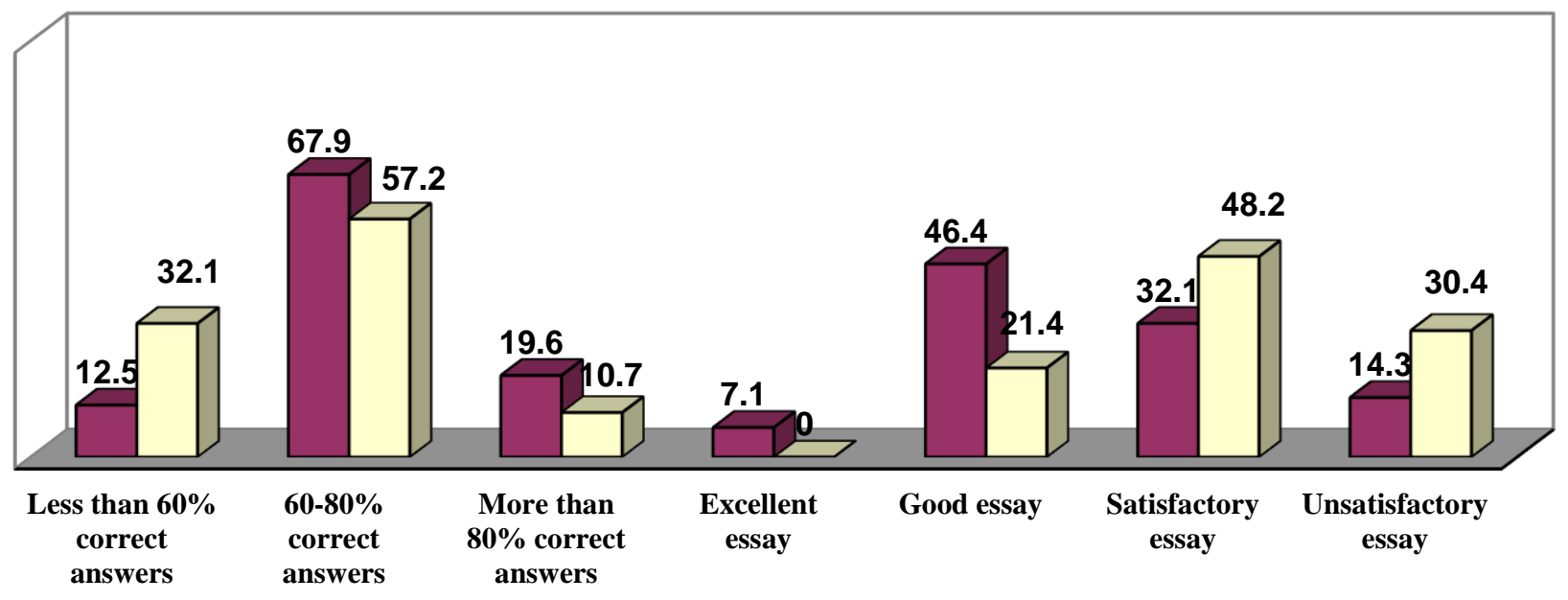

\section{$\square$ Number of postgraduates in experimental group $\square$ Number of postgraduates in control group}

Figure 20. The results of the second ascertaining test

Based on the results of the ascertaining section, the following conclusions were made:

1. Postgraduates as a whole possess the skills of oral and written speech sufficiently to conduct an experiment on the formation of professional communicative competence at the postgraduate stage.

2. The greatest difficulty during the interview was caused by the need to solve problematic issues. Apparently, at this stage of proficiency in a foreign 
language, the postgraduates were not ready for this style of communication, they basically switched to retelling prepared thematic texts, and the unexpected remarks of the teacher conducting the dialogue were either ignored or were not able to argue.

3. Checking the essay showed that the main difficulties in writing can be attributed to the area of content and composition, since even the existing interesting ideas and thoughts were not sufficiently reasoned, logically substantiated and coherently designed. The volume of vocabulary and grammatical correctness, on the contrary, did not present any particular difficulties.

4. Thus, it was concluded that special attention should be paid to the development of skills of foreign language communicative competence (critical thinking, the logical design of one's own thoughts in English, the ability to listen and hear the interlocutor and respond adequately, the ability to abstract and annotate a special text of any complexity), which is the basis of the communication process, both orally and in writing, especially in the scientific environment. The development of a foreign language communicative competence should take place on the basis of professionally significant vocabulary, reflecting the specifics of the specialty.

The study and analysis of literary sources on the problem of research, work experience and the results of the ascertaining section determined the content and technology of the formation of foreign language communicative competence of lawyers at the postgraduate stage.

\section{Experimental learning}

An important condition for ensuring the successful course of experimental training was the compliance of its conduct with the following principles:

- consistency (from the known to the unknown, from simple to complex, from small to large, from the acquisition of skills to their application, etc.);

- cyclicality (regular return to known materials and skills and training them at a higher quality level using new acquired knowledge);

- complexity (integration of aspects, types of speech activity; application of TCO; change of types of work - individual, pair, in small groups, etc.; change of roles - listeners, speakers, interlocutors, arbitrators, etc.);

- discipline (high pace of training, clarity and consistency in the implementation of the set goals).

- interdependence (ensuring the overall success of the group based on the degree of independent preparation of both the master's student and the teacher).

- control (unconditional observance of objectivity). 
A sufficient level of knowledge of English grammar among the master's students of the experimental and control groups made it possible to put into practice the objectives and goals of the program.

The materials used in the learning process were selected and organized taking into account general and specific criteria.

Teaching methods. The creation of the most favorable psychological microclimate in the group was ensured by constructive, friendly relationships between teachers and the group, based on trust and respect for each other; an objective attitude towards all postgraduates; discipline, obligatory for everyone, and a sense of humor. When forming groups, it is necessary to take into account the interpersonal relationships between postgraduates, their typological characteristics and cognitive styles. The advantage of group work is that it ensures the participation in the discussion of all postgraduates, without exception, regardless of their regalia and age. By the time the training experiment began, the teacher already knew about the personality types of all subjects, about their preferred learning strategies, about relationships with each other, and, importantly, about the topics of scientific research of postgraduates.

In the course of the research, first of all, the criteria and indicators of the formation of foreign language communicative competence were identified.

The criteria were the formation of the language competence of lawyers, which is determined;

1) the level of proficiency in grammatical and linguistic units;

2) the ability to evaluate, fix the position of the interlocutor (or opponent) in communication;

3 ) the ability to evaluate and maintain their own position in communication;

4) abilities and skills to oppose.

Further, according to each criterion, tables are attached, reflecting the dynamics of the formation of the foreign language communicative competence of lawyers at the experimental stage of training (Tables $8,9,10,11$ ).

Table 8. The proficiency in grammatical and linguistic units (criterion 1):

\begin{tabular}{|l|c|c|c|}
\hline \multirow{2}{*}{ Experiment stages } & \multicolumn{3}{|c|}{ Proficiency level (number and \%) } \\
\cline { 2 - 4 } & High & Middle & Low \\
\hline \multicolumn{4}{|c|}{ Experimental group } \\
\hline 1. Beginning 56/100 & $15 / 26,8 \%$ & $16 / 28,6 \%$ & $25 / 44,6 \%$ \\
\hline 2. Halfway 56/100 & $16 / 28,6 \%$ & $17 / 30,4 \%$ & $23 / 41,0 \%$ \\
\hline 3. End 56/100 & $24 / 42,8 \%$ & $19 / 34.0 \%$ & $13 / 23,2 \%$ \\
\hline
\end{tabular}




\begin{tabular}{|l|c|c|c|}
\hline \multicolumn{4}{|c|}{ Control group } \\
\hline 1. Beginning 56/100 & $14 / 25,0 \%$ & $19 / 34.0 \%$ & $23 / 41,0 \%$ \\
\hline 2. Halfway 56/100 & $16 / 28,6 \%$ & $18 / 32,1 \%$ & $22 / 39,3 \%$ \\
\hline 3. End 56/100 & $20 / 35.7 \%$ & $16 / 28.6 \%$ & $20 / 35,7 \%$ \\
\hline
\end{tabular}

The second criterion, characterizing the formation of the ability to assess and fix the position of the interlocutor (or opponent) in communication, is manifested in such indicators as the ability to highlight the semantic parts and the logic of constructing the interlocutor's text; the ability to correlate the speech of the interlocutor with the subject and purpose of the conversation; the ability to assess the sufficiency and reliability of the argumentation, the ability to understand the intention of the interlocutor.

Table 9. The proficiency in evaluating and committing to the opponent's position in communication (criterion 2):

\begin{tabular}{|l|c|c|c|}
\hline \multirow{2}{*}{ Experiment stages } & \multicolumn{3}{|c|}{ Proficiency level (number and \%) } \\
\cline { 2 - 4 } & High & Middle & Low \\
\hline \multicolumn{4}{|c|}{ Experimental group } \\
\hline 1. Beginning 56/100 & $15 / 26,8 \%$ & $21 / 37,5 \%$ & $20 / 35,7 \%$ \\
\hline 2. Halfway 56/100 & $16 / 28,6 \%$ & $23 / 41,0 \%$ & $17 / 30,4 \%$ \\
\hline 3. End 56/100 & $26 / 46,4 \%$ & $19 / 34.0 \%$ & $11 / 19,6 \%$ \\
\hline \multicolumn{4}{|c|}{ Control group } \\
\hline 1. Beginning 56/100 & $15 / 26.8 \%$ & $17 / 30.4 \%$ & $24 / 42,8 \%$ \\
\hline 2. Halfway 56/100 & $16 / 28,6 \%$ & $17 / 30,4 \%$ & $23 / 41,0 \%$ \\
\hline 3. End 56/100 & $17 / 30.4 \%$ & $18 / 32.1 \%$ & $21 / 37.5 \%$ \\
\hline
\end{tabular}

The third criterion characterizing the formation of the ability to assess and maintain one's own position in communication is manifested in the following indicators: the ability to clearly, concisely and logically formulate one's own position; the ability to argue their speech (the adequacy of the text to the topic of discussion and the position taken in communication; variety and evidence of arguments).

Table 10. The proficiency in evaluating and committing to own position in communication (criterion 3):

\begin{tabular}{|c|c|c|c|}
\hline \multirow{2}{*}{ Experiment stages } & \multicolumn{3}{|c|}{ Proficiency level (number and \%) } \\
\cline { 2 - 3 } & High & Middle & Low \\
\hline \multicolumn{3}{|c|}{ Experimental group } \\
\hline
\end{tabular}




\begin{tabular}{|l|c|c|c|}
\hline 1. Beginning 56/100 & $12 / 21,4 \%$ & $17 / 30,4 \%$ & $27 / 48,2 \%$ \\
\hline 2. Halfway 56/100 & $19 / 34.0 \%$ & $26 / 46,4 \%$ & $11 / 19,6 \%$ \\
\hline 3. End 56/100 & $27 / 48,2 \%$ & $22 / 39,3 \%$ & $7 / 12,5 \%$ \\
\hline \multicolumn{4}{|c|}{ Control group } \\
\hline 1. Beginning 56/100 & $11 / 19,6 \%$ & $19 / 34.0 \%$ & $26 / 46,4 \%$ \\
\hline 2. Halfway 56/100 & $17 / 30,4 \%$ & $16 / 28,6 \%$ & $23 / 41,0 \%$ \\
\hline 3. End 56/100 & $18 / 32.1 \%$ & $17 / 30.4 \%$ & $21 / 37.5 \%$ \\
\hline
\end{tabular}

The fourth criterion, characterizing the development of skills to oppose, is manifested in such indicators as the ability to ask questions (clarifying and leading); the ability to use the arguments of the interlocutor in polemics without allowing a dispute; the ability to reach a general conclusion.

Table 11. The proficiency in opposing (criterion 4):

\begin{tabular}{|l|c|c|c|}
\hline \multirow{2}{*}{ Experiment stages } & \multicolumn{3}{|c|}{ Proficiency level (number and \%) } \\
\cline { 2 - 4 } & High & Middle & Low \\
\hline \multicolumn{4}{|c|}{ Experimental group } \\
\hline \multicolumn{4}{|c|}{3} \\
\hline 1. Beginning 56/100 & $14 / 25,0 \%$ & $17 / 30,4 \%$ & $25 / 44,6 \%$ \\
\hline 2. Halfway 56/100 & $18 / 32.1 \%$ & $21 / 37.5 \%$ & $17 / 30.4 \%$ \\
\hline 3. End 56/100 & $24 / 42.8 \%$ & $22 / 39.3 \%$ & $10 / 17.9 \%$ \\
\hline \multicolumn{4}{|c|}{ Control group } \\
\hline 1. Beginning 56/100 & $14 / 25.0 \%$ & $20 / 35.7 \%$ & $22 / 39.3 \%$ \\
\hline 2. Halfway 56/100 & $15 / 26.8 \%$ & $20 / 35.7 \%$ & $21 / 37.5 \%$ \\
\hline 3. End 56/100 & $16 / 28.6 \%$ & $20 / 35.7 \%$ & $20 / 35.7 \%$ \\
\hline
\end{tabular}

Diagnostics of the formation and improvement of the foreign language communicative competence of lawyers at the postgraduate stage, as well as the quantitative and qualitative results of the educational activities of the trained experimental groups showed a number of positive changes. So, already at the second stage, most of the postgraduates of the experimental groups showed the ability to fix, argue and maintain their own position in the process of professionally-oriented communication, the ability to formulate clear conclusions, the ability to communicate in specified forms of organizing statements, the ability and skills to oppose. 
Analysis of the data obtained indicates that the quantitative indicators of the formation of the ability to assess and fix the position of the interlocutor (or opponent) in communication (criterion 2) and the formation of skills to oppose (criterion 4) coincide.

A direct cut was made at the end of the experimental training. The purpose of the direct cut was, firstly, to assess the success of postgraduates mastering the experimental groups of professional communication skills. Secondly, due to the fact that in the process of experimental training, a large amount of additional information material was worked out, the cut involved an assessment of the knowledge of theoretical material according to thematic series (knowledge of the legal norms of Great Britain and the USA, knowledge of differences in the legal norms of countries (within the framework of the passed material. Postgraduates were offered two types of assignments.

As a control test, the specific situations "The questioning and the Indictment" or "Corruption. The ways of combatting it" were suggested for postgraduates. A simple algorithm of work was assumed: it was necessary to highlight the significant structural components in the text, highlight and describe the problem, offer a reasoned solution, annotate the sources and highlight relevant information, organize ideas in an outline, attribute ideas to the sources and write references, write research questions.

Oral statements of postgraduates were recorded on video, after which they were processed. Their analysis led to the conclusion that more often postgraduates use abstracting schemes and cliches studied in the classroom.

The second task involved the performance of a written test, the correct performance of which allowed assessing the level of assimilation of language and theoretical material in a thematic series.

The results of the analysis testify to the effectiveness of the process of forming professional communicative competence in the process of teaching the language of the specialty of postgraduate lawyers.

At the third stage of the experiment, the degree of formation of foreign language communicative competence and the level of stability of the formed skills and abilities were checked.

Master's students with a high, but unstable level of foreign language communicative competence formation applied the acquired skills only in classes in the language of their specialty and only in those forms of work that were worked out during experimental training (solving problem tasks, analyzing specific situations, business game "Discussion") ...

Postgraduates with a low level of professional communication skills and abilities avoided participation in collective forms of work, had a weak level of 
linguistic and intellectual competence, had psychological problems in communicating with others (including communication in their native language).

Qualitatively assessing the statements of postgraduates in terms of the level of mastering foreign language communication skills and abilities, we can note as indicative the fact that the positive results recorded during the experiment were also noted by the teachers of related departments.

An analysis of the results of a questionnaire survey among teachers of related departments showed that $72 \%$ of respondents note a general increase in the culture of speech in applicable language among postgraduates in such aspects as consistency, clarity, argumentation, brevity of statements, that is, there is a "reverse transfer" of formed knowledge and skills.

Were identified and confirmed the conditions that affect the effectiveness of the process: the use of a problematic approach in training; reliance on pre-formed linguistic competence; inclusion in the educational process of purposeful activities to improve language competence; phased formation of foreign language communication skills and abilities; organization of a purposeful, controlled, systemic process of the formation of foreign language communication skills; modern approaches to the content and methods of teaching foreign language communicative competence.

Analysis of the results of experimental training. The experimental work was quite effective: there was an increase in the number of postgraduates with a high and medium level of foreign language communicative competence formation.

The research data indicate that the experimental work was quite effective: its result was an increase in the number of postgraduates with a high level of formation of foreign language communicative competence:

- in the first criterion, it grew by $16.0 \%$;

- in the second case - by $19.6 \%$;

- in the third - by $26.8 \%$;

- in the fourth - by $17.8 \%$ (compared the initial and final indicators of the high level of the experiment).

The data of the transforming stage indicate that the highest indicators, stating the level of formation of foreign language communicative competence, were recorded by us at the third stage of diagnosis (Table 12, Figures 21 and 22).

The obtained quantitative data were also confirmed by the results of observations of the activities of postgraduates in the classroom in other disciplines, in terms of interaction with teachers and fellow students at the final stage of the experimental work, which testified to its productivity. 
The results of experimental training.

1) All of the above confirms our earlier assumption that the work system we propose to a large extent activates the mental activity of trainees, allows them to develop and improve professional communication skills and abilities, to carry out work within the framework of an interdisciplinary approach.

2) Without exerting a negative impact on the mastery of language material within the thematic series, the proposed path of training leads to the fact that lawyers at the postgraduate stage of preparation master foreign language communication skills and abilities. 
Table 12. Dynamics of the development of foreign language communicative competence of postgraduates of Law

\begin{tabular}{|c|c|c|c|c|c|c|c|c|c|}
\hline \multirow{3}{*}{$\begin{array}{l}\text { Component } \\
\text { manifestation } \\
\text { criteria }\end{array}$} & \multicolumn{9}{|c|}{ Level } \\
\hline & \multicolumn{3}{|c|}{$\begin{array}{c}1^{\text {st }}-4^{\text {th }} \\
\text { thematic series }\end{array}$} & \multicolumn{3}{|c|}{$\begin{array}{c}5^{\text {th }}-8^{\text {th }} \\
\text { thematic series }\end{array}$} & \multicolumn{3}{|c|}{$\begin{array}{c}9^{\text {th }}-10^{\text {th }} \\
\text { thematic series }\end{array}$} \\
\hline & low & middle & high & low & middle & high & low & middle & high \\
\hline $\begin{array}{l}\text { The proficiency in } \\
\text { grammatical and } \\
\text { linguistic units }\end{array}$ & $25 / 44.6$ & $16 / 28.6$ & $15 / 26.8$ & $23 / 41.0$ & 17 / 30.4 & $16 / 28.6$ & $13 / 23.2$ & $19 / 34.0$ & $24 / 42.8$ \\
\hline $\begin{array}{l}\text { The proficiency in } \\
\text { evaluating and } \\
\text { committing to the } \\
\text { opponent's } \\
\text { position in } \\
\text { communication }\end{array}$ & $20 / 35.7$ & $21 / 37.5$ & $15 / 26.8$ & $17 / 30.4$ & $23 / 41.0$ & $16 / 28.6$ & $11 / 19.6$ & 19 / 34.0 & $26 / 46.4$ \\
\hline $\begin{array}{l}\text { The proficiency in } \\
\text { evaluating and } \\
\text { committing to own } \\
\text { position in } \\
\text { communication }\end{array}$ & $27 / 48.2$ & $17 / 30.4$ & $12 / 21.4$ & $11 / 19.6$ & $26 / 46.6$ & $19 / 34.0$ & $7 / 12.5$ & $22 / 39.3$ & $27 / 48.2$ \\
\hline $\begin{array}{l}\text { The proficiency in } \\
\text { opposing }\end{array}$ & $25 / 44.6$ & $17 / 30.4$ & $14 / 25.0$ & 17 / 30.4 & $21 / 37.5$ & $18 / 32.1$ & $10 / 17.9$ & $22 / 39.3$ & $24 / 42.8$ \\
\hline
\end{tabular}


口Criterion $1 \quad \square$ Criterion $2 \quad \square$ Criterion $3 \quad \square$ Criterion 4

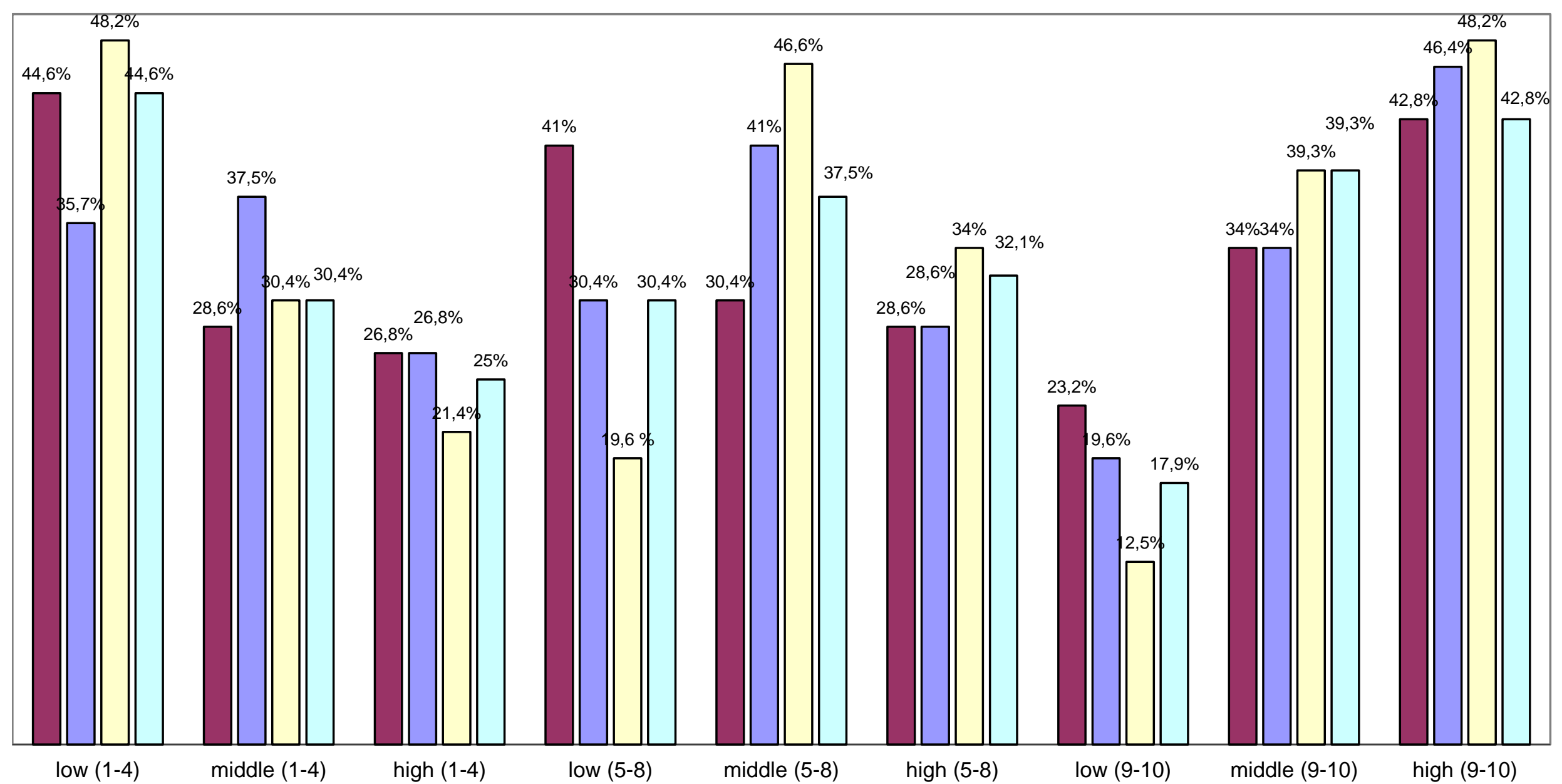

Fig. 21. Dynamics of the development of foreign language communicative competence 


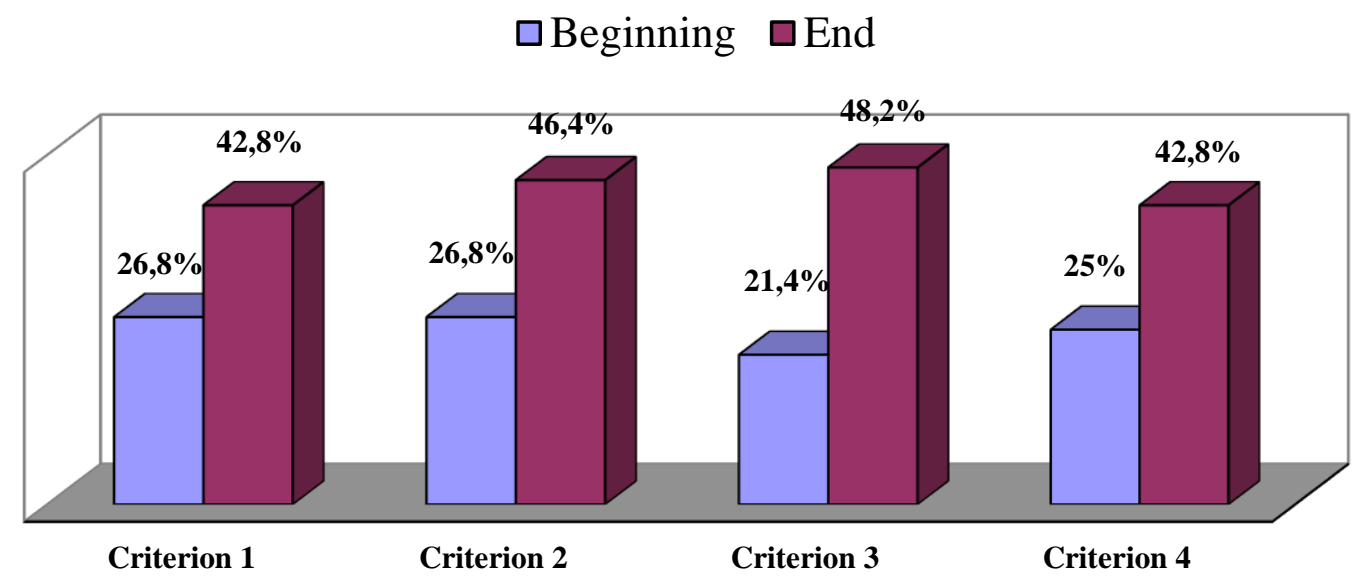

Fig. 22. Dynamics of the number of postgraduates with a high level

Proficiency in foreign language communicative competence

So, the experimental data clearly confirm our hypothesis that the effectiveness of the formation of the foreign language communicative competence of lawyers studying at the university of the law enforcement system in the specialty 6N0303 - Law enforcement, increases if the developed by us method of formation communicative competence. 


\section{CONCLUSION}

The state of the training of specialists in the field of jurisprudence is determined by the fact that it involves a revision of traditional methods and techniques of teaching a shift in emphasis in teaching to practical training, to the ability to navigate in the changing conditions of scientific and professional activity. The revision of the methods and techniques of teaching lawyers should be guided by the theoretical position of linguistics according to which the language of law is defined as a sublanguage, i.e. on the recognition of the obviousness of the inextricable link between language and jurisprudence.

Integration processes with the world community determine the actualization of foreign language training for lawyers in general, and in the system of postgraduate education.

The goal of postgraduate foreign language teaching is the formation of a foreign language communicative competence which involves the activation of linguistic, subject, pragmatic competencies.

The social significance of the foreign language communicative competence of lawyers lies in the fact that it is one of the factors of their competitiveness in modern conditions of expanding international cooperation in the field of law enforcement and lawmaking.

The formation of the foreign language communicative competence of lawyers in the system of postgraduate education should be carried out taking into account the culture-forming concept of teaching foreign languages, the principles of pedagogical hermeneutics, the common European levels of language competence, the idea of problem-based learning.

Thus, the identified positive trends in the formation of foreign language communicative competence in the system of postgraduate education indicate the need for further development of the problem both at the theoretical and practical levels. The development of methodological support for the formation of foreign language communicative competence of postgraduates of all non-linguistic specialties is subject to further research which in our opinion, involves the creation of "Language for specific purposes" course for students in the postgraduate education system and a number of thematic special courses focused on optimizing interdisciplinary connections and professional communication skills. 


\section{REFERENCES}

Abdrasulov E. B. \& Seytaeva Zh.S. (2016). Issues of improvement of educational activities for training masters and doctors $(\mathrm{PhD})$ in special educational institutions of the Republic of Kazakhstan. Bulletin of the Academy of Law enforcement. Pedagogy and methods of postgraduate education bulletin of the Academy of Law enforcement bodies. No. 1, p. 145.

Abdulrahman, N., Emad A.S.Abu-Ayyash (2019). Linguistic competence, Communicative Competence and Interactional Competence. Journal of Advances in Linguistics Vol 10 (2019) ISSN: 2348-3024. https://doi.org/10.24297/jal.v10i0.8530

Abulhanova - Slavskaya K.A. (1980). Deyatelnost I psihologiya lichnosti [Activity and personality psychology]. Moscow: p. 187 [in Russian].

Abulkhanova-Slavskaya K.A. (1980). Deyatelnost $i$ psikhologiya lichnosti. [Activity and personality psychology]. Moscow [in Russian].

Anderson, L. W., \& Krathwohl, D. R. (2001). A taxonomy for learning, teaching, and assessing, Abridged Edition. Boston, MA: Allyn and Bacon

Arthur L., Brennan J. \& de Weert E. (2007). Employer and higher education perspectives on graduates in the knowledge society. A report from the European Commission Framework VI project: "The Flexible Professional in the Knowledge Society". Retrieved from http:/www.open.ac.uk/cheri/pages/ CHERI-Reports-2007.shtml

Avanesov Yu.A. \& KarasL. Yu. (1996). Referat-daydzhest uchebnika. Meskona M. KH, Alberya M., Hedouri F. «Osnovy menedzhmenta» [Abstract - digest of the textbook by M. Kh. Meskon, M. Alberya, F. Hedouri "Fundamentals of Management"]. Moscow: Lux-art, pp 296, 150 [in Russian].

Azimov E. G. \& Shchukin A. N. (2009). Novyi slovar metodicheskikh terminov $i$ ponyatiy (teoriya I praktika obucheniya yazykam) [New dictionary of methodological terms and concepts (theory and practice of teaching languages)]. - Moscow: Izdatelstvo IKAR [in Russian].

Babenko A.P. (1968). Organizatsiya raboty nad nekotorymi vidami ekspressivnoy rechi na starshikh kursakh yazykovogo fakulteta. [Organization of work on some types of expressive speech at the senior years of the language faculty]. Extended abstract of Candidates thesis. Moscow: n.p. [in Russian].

Bakhtin M. M. (1976). Problema teksta (opyt filosofskogo analiza) [The problem of text (experience of philosophical analysis)]. Voprosy literatury. N.p. Vol 10, pp. 125 - 126 [in Russian].

Bakhtin M.M. (1986). Estetika slovesnogo tvorchestva [Aesthetics of verbal creativity], Moscow, p. 10, [in Russian]. 
Balli Sh. (2003). Yazyk I zhizn [Language and life]. Geneva Linguistic School, Transl. from French, Moscow: Editorial URSS, p. 232 [in Russian].

Barthold, L. S. (2010). Gadamer's Dialectical Hermeneutics, Lanham, MD: Lexington, 2010.

Bazarov T.U. (n.d.). Kompetentsii buduchshego: Kvalifikatsiya? Kompetentnost (kriterii kachestva)? [Competencies of the future: Qualifications? Competence (quality criteria)?]. Retrieved from http: www.tltsu.ru/publectures/lecture06.html

Belyaev A.S. (1997). Obucheniye inoyazychnomu professionalno oriyentirovannomu rechevomu obshcheniyu s uchetom spetsifiki fizkulturnykh vuzov [Teaching foreign language professionally oriented speech communication taking into account the specifics of physical culture universities]. Extended abstract of Doctor's thesis. Saint Petersburg, p.190, 19 [in Russian].

Berdichevsky A.P. (2002). Sovremennyye tendentsii v obuchenii inostrannomu yazyku $v$ Yevrope [Modern trends in teaching a foreign language in Europe]. Russian language abroad: № 2, pp. 3-5 [in Russian].

Berkalova O.I. (1989). Sootnosheniye dialoga I monologa $v$ ustnoy, pismennoy rechi v grazhdanskom sudoproizvodstve [The ratio of dialogue and monologue in oral, written speech in civil proceedings]. Extended abstract of Candidate'sthesis. Moscow: Moscow State University, pp. 6, 23 [in Russian].

Beswick, D. (2017). Cognitive motivation. [From curiosity to identity, purpose and meaning]. Cambridge University Press, DOI: https://doi.org/10.1017/978131 6822920.015. pp 317-368

Bezrukova B.S. (1996). Slovar novogo pedagogichevskogo myshleniya [Dictionary of New Pedagogical Thinking]. Ekanerinburg: Alternative pedagogy, p.94 [in Russian].

Bim T.L. (1977). Metodika obucheniya inostrannym yazykam kak nauka I problem shkolnogo uchebnika: opyt sistemno-strukturnogo opisaniya [Methodology of teaching foreign languages as a science and problems of a school textbook: the experience of a system-structural description]. - Moscow: Education, p. 40,19 [in Russian].

Bolotov V.A. \& Serikov V.V. (2003). Kompetentnostnaya model: ot idei $k$ obrazovatelnoi programme [Competence model: from idea to educational program]. N.p.: \# 10, p. 12 [in Russian].

Brennan, A.M. (2019). Transnational Terrorist Groups and International Criminal Law. ISBN 9780367588106, Published June 30, 2020 by Routledge, 232 Pages.

Brooks N. (1997). Teaching Culture in the Foreign Language Classroom IN HEUSINKVELD. P. R. (Ed.) Pathways to Culture. Yarmouth. ME: Intercultural Press. 
Brown, D. (2000). Principles of language learning and teaching. (4th ed.) White Plains, NY: Longman.

Brown, D. (2001). Teaching by principles. (2th ed.) White Plains, NY: Longman.

Brown H.D. (2000). Principles of Language Learning and Teaching. NY: Longman.

Campbell, B., Kaunda, L., Allie, S., Buffler, A., \& Lubben, F. (2000). The communication of laboratory investigations by university entrants. Journal of Research in Science Teaching, 37, 839-853.

Cat J. (2017). The Unity if Science. [The Stanford Encyclopedia of Philosophy. Center for the Study of Language and Information (CSLI)], Stanford University, Library of Congress catalog data: ISSN 1095-5054 https://plato.stanford.edu/entries/scientific-unity/

Chen X. (2013). An Empirical Study of the Influence of PBL Teaching Mode on Critical Thinking Ability of Non-English Majors. J. PLA Univ. Foreign Lang. 36:68-72.

Chulkova V.S. (1980). Problema integratsii nauchnogo teksta. Funktsionalnyi stil nauchnoy prozy. Problemy lingvistiki I metodiki prepodavaniya [The problem of integrating a scientific text. Functional style of scientific prose. Problems of linguistics and teaching methods]. Moscow: Nauka, p. 36 [in Russian].

Common European Framework of Reference for Languages: Learning, Teaching, Assessment, Language Policy Unit, (2000) Table 1. Common Reference Levels: global scale, Neil Jones, Marianne Hirtzel, University of Cambridge Local Examinations Syndicate. Strasbourg, www.coe.int/lang-CEFR, p.24

DeBoer, G. E. (2000). Scientific literacy: Another look at its historical and contemporary meaning and its relationship to science education reform. Journal of Research in Science Teaching, 37, 582-601.

Decree of the Government of the Republic of Kazakhstan (2012) No. 1080 "On the approval of state compulsory education standards of the corresponding levels of education" // Information and legal system of regulatory legal acts of the Republic of Kazakhstan "Adilet" http://adilet.zan.kz/rus/ docs / P1200001080 \# z127.

Decree of the President of the Republic of Kazakhstan (1998). O Gosudarstvennoy programme funktsionirovaniya I razvitiya yazykov [On the State Program for the Functioning and Development of Languages] No. 4106 [in Russian].

Ding X.W. (2016). The Effect of WeChat-Assisted Problem-Based Learning on the Critical Thinking Disposition of EFLLearners. Int. J. Emerg. Retrieved from doi:10.3991/ijet.V11i12.5927.

Dilthey V. (1992). Descriptive and analytical psychology. Moscow. 
Dottori, R. (ed.) (2012). Fifty years after H.-G. Gadamer's 'Truth and Method': Some considerations on H.-G. Gadamer's main philosophical work, Berlin: LIT Verlag.

Dulov A.V. (1975). Sudebnaya psikhologiya [Forensic psychology]. Minsk: Vysheyshaya shkola, p. 39 [in Ukranian].

Efroimson V.P. \& Izumov E.A. (2009). Na chto my nadeemsya, ili nuzhno li rastit geniev? [What do we hope for or is it necessary to raise geniuses?]. Moscow: Politizdat, p. 560 [in Russian].

Encyclopedia of Private International Law (2017). Edited by Jürgen Basedow, Giesela Rühl, Franco Ferrari and Pedro de Miguel Asensio https://doi.org/10.4337/9781782547235

Epifanova S. (2000). Formirovaniye uchebnoy motivatsi[Formation of educational motivation]. Vyssheye obrazovaniye $\mathrm{v}$ Rossii [Higher education in Russia], p.106 [in Russian].

Facione P. (1992). Critical Thinking Disposition as a Measure of Competent Clinical Judgment: The Development of the California Critical Thinking Disposition Inventory. The California Academic Press; Millbrae, CA, USA. Journal of Nursing Education. 33(8):345-350. https://doi.org/10.3928/01484834-19941001-05

Faenova M.O. (1991). Obucheniye culture obshcheniya na angliyskom yazyke: Nauchno - teoreticheskoye posobiye [Teaching a culture of communication in English: Scientific and theoretical manual], Moscow: Vysshaya shkola, p.7, [in Russian].

Fernández-Salinero C. (2006). "Las competenciasen el marco de la convergenciaeuropea: Un nuevo concepto para el diseño de programaseducativos". Encounters on Education, Volume 7, pp. 131-153.

Flinders D.J. (2015). Curriculum and Teaching Dialogue (CTD) Vol.17

Folsom F. (1977). Book about language. Moscow: Progress, p. 160.

Freire P. (1972). Pedagogy of the oppressed. New York: Herder and Herder, p. 162.

Frey A., Balzer L.\& Renold U. (2002). Soziale kompetenz und persönlichkeit: zusammenh ängezwischen sozialer kompetenz und den Big Five der person lichkeitbeijun generwachsenen. New York: Basic Books. Retreated from ISBN 393733959, 9783937333953

Fumadel M. (1976). Metodika prepodavaniya inostrannykh yazykov za rubezhom [Methods of teaching foreign languages abroad]. Moscow: Progress, p. 153, 48 -51 [in Russian].

Galperin I. R. (1988). Ocherki po stilistike angliyskogo yazyka [Essays on the style of the English language]. Moscow: Publishing house of literature on foreign lang., p. 459 [in Russian]. 
Garvey, W. D., \& Griffith, B. C. (1972). Communication and information processing within scientific disciplines: Empirical findings for psychology. Information Storage and Retrieval, 8, 123-126.

Gez N. I. (1993). Obucheniye govoreniyu [Teaching to speak]. Text of lectures for the course "Methods of teaching foreign languages. Moscow: MGPIIYa, p.93[in Russian].

Gill, E. (2020). What is your teaching style? Resilient Educator.com/styles

Golev N.D. (1999). Yuridicheskiy aspect yazyka v lingvisticheskom osveshchenii [Legal aspect of language in linguistic illumination]. Barnaul: Jurisingvistics-1: Problems and prospects, pp. 11-58 [in Russian].

Goncharova N.L. (2007). Kategorii "kompetentnost" I "kompetentsiya" $v$ sovremennoi obrazovatelnoi paradigm [Categorie "competence" in the modern educational paradigm]. North Caucasus: Collection of scientific papers, № 5. Retrieved from http: // www.nestu.ru. [in Russian].

GOSO RK 3.09 .153 (2004). Gosudarstvenny yobshcheobyazatelnyy standard obrazovaniya Respubliki Kazakhstan. Magistratura. Spetsialnost 6N0303 Pravookhranitelnaya deyatelnost. [State compulsory education standard of the Republic of Kazakhstan. Master's degree. Specialty 6N0303 - Law enforcement]. Astana: MES RK [in Russian].

Gosudarstvennaya programma razvitiya obrazovaniya I nauki Respubliki Kazahstan na 2020 -2025 gody (2019). [Government program of development of education and science of the Republic of Kazakhstan for 2020 - 2025]. Approved by government decree of the Republic of Kazakhstan, dated December 27, 2019 No. 988. Retrieved from http://www.ecaqa.org/doxs/gosudarstvennaya -programma-razvitiya-obrazovaniya-i-nauki-respubliki-kazahstan.[in Russian].

Grazhdanskiy kodeks Respubliki Kazakhstan (ot 27 dekabrya 1994 g.) (Obshchaya chast') (s izmeneniyami, vnesennymi Ukazami Prezidenta, imeyushchimi silu zakona, ot 31.08.1995 g.; ot 05.10.1995 g. N 2489; ot 27.01.1996 g. № 2835 I Zakonami $R K$ ot15.07.1996 g. № 30-1; ot 07.12.1996 g. № 50-1; ot 21.01.1997 g. № 68-1; ot 05.03.1997 g. № 80-1; ot 19.06.1997 g. № 132-1; ot 19.06.1997 g. № 134-1; ot 02.07.1997 g. № 144-1; ot 11.07.1997 g. № 154-1; ot 02.03.1998 g. № 211-1; ot 22.04.1998 g. № 221-1; ot 29.07.1998 g. № 2381; ot 10.07 .1998 g. № 282-1; ot 10.07 .1998 g. № 283-1; ot 16.12 .1998 g. № 320-1; ot 16.07.1999 g. № 436-1; ot 16.07.1999 g. № 440-1; ot 04.11 .1999 g. № 472-1; ot 08.11.2000 g. № 96-II; ot 18.12.2000 g. № 128-II; ot 15.01.2001 g. № 141-II; ot 02.03.2001 g. № 162-II; ot 11.07.2001 g. № 239II) [Civil Code of the Republic of Kazakhstan dated December 27, 1994 (General part) (as amended by Presidential Decrees having the force of law, dated 08.31.1995; dated 05.10.1995, No. 2489; dated 27.01.1996, No. 2835 and the Laws of the RK dated 15.07.1996, No. 30-1; dated 07.12.1996, No. 50-1; dated 21.01.1997, No. 68-1; dated 05.03.1997, No. 80-1; dated 19.06. 1997 No. 
132-1; dated 19.06.1997, No. 134-1; dated 02.07.1997, No. 144-1; dated 11.07.1997, No. 154-1; dated 02.03.1998, No. 211- 1; dated 22.04.1998, No. 221-1; dated 29.07.1998, No. 238-1; dated 10.07.1998, No. 282-1; dated 10.07.1998, No. 283-1; dated 16.12.1998 No. 320-1; dated July 16, 1999, No. 436-1; dated July 16, 1999, No. 440-1; dated November 4, 1999, No. 472-1; dated November 8, 2000, No. 96-II ; dated 18.12.2000, No. 128-II; dated 15.01.2001, No. 141-II; dated 02.03.2001, No. 162-II; dated 11.07.2001, No. 239-II)]. [in Russian].

Greenberg K. L. (1988). Assessing writing: Theory and practice. In J.H. McMillan (Ed.), New Directions for Teaching and Learning: №. 34. San Francisco.

Grishina O. N. (1982). Sootnosheniye povestvovaniya, opisaniya I rassuzhdeniya $\mathrm{v}$ khudozhestvennom tekste (na materiale angliyskoy I amerikanskoy prozy XX veka) [Correlation of narration, description and reasoning in a literary text (based on the material of English and American prose of the 20th century)]. Extended abstract of candidates thesis [in Russian].

Gubayeva T. V. (1995). Slovesnost v yurisprudentsii [Literature in jurisprudence]. Kazan: Publishing house of the Kazan state University, pp 8-9, 220 [in Russian].

Hamdan, A. R.; Kwan Chan Li; Khan Aqeel; Ghafar Mohamed Najib Abdul; \& Sihes Ahmad Johari (2014). Implementation of Problem Based Learning among Nursing Students. International Education Studies, Vol. 7, n7, pp.136-142. Retrieved from Web sitehttp://www.ccsenet.org/journal/index.php/es

Harir Foroush and Sadeghi (1389 / 2010). Teaching pattern of brainstorming.

Hassall T.\& Dunlop A. (1996). Internal audit education: exploring professional competence. Managerial Auditing Journal. Vol. 11., № 5., pp. 7-49.

Haupt C. (2016). Professional speech. The Yale Law Journal. Retrieved from: https://www.yalelawjournal.org/pdf/b.1238.Haupt.1303_hen9z6bg.pdf

Heidegger's ways (1994). Trans. by John W. Staley, Albany, NY: SUNY Press.

HoustonW.R.\& Howsam R.B. (Ed.). (1972). Competency-Based Teacher Education: Progress, Problems and Prospects. Chicago: Science Research Association, Vol. X, p. 182.

Hutchinson T.\& Waters A. (1987). English for Specific Purposes. A learningcentered approach. Cambridge: Cambridge University Press.

Hutorskoi A.V. (1998). Evristicheskoe obuchenie [Heuristic learning]. Moscow: MPA, p.266 [in Russian].

Hye Sun You (2017). Why Teach Science with an Interdisciplinary Approach: History, Trends, and Conceptual Frameworks. Journal of Education and Learning. Vol. 6, No.4. http:// www.ccsenet.org/journal/index.php/jel. https://doi.org/10.5539/jel.v6n4p66. 
IBA International Principles on Conduct for the Legal Profession. (2011). London: International Bar Association

Ilyichev L.F, Fedoseev P.N. \& Kovalev S.M. (1983). Filosofskii entsyclopedicheskii slovar [Philosophical Encyclopedic Dictionary]. Moscow: Soviet Encyclopedic dictionary, p. 840 [in Russian].

Isaeva T.E. (N.d.). Klassifikatsiya professionalno - lichnostnyh kompetentsii vuzovskogo prepodavatelya [Classification of professional and personal competencies of a university teacher]. Proceedings from "Proceedings of the international scientific and practical Internet conference "Higher education teacher in the XXI century". Rostov - na- Donu: Vol. 4 pp. 15 -21 [in Russian].

Isaeva T.E. (2003). Pedagogicheskaya kultura prepodavatelya kak uslovie I pokazatel kachestva obrazovatelnogo protsessa v vysshei shkole (Sravnitelnyi analiz otechestvennogo I mirovogo obrazovatelnogo protsessa) [The pedagogical culture of the teacher as a condition and indicator of the quality of the educational process in higher education (Comparative analysis of the domestic and world educational process)]. Rostov na Donu: p. 312 [in Russian].

Iskandarova O. Yu. (1997). Problemno-kommunikativnyi podkhod kak factor aktivizatsii poznavatelnoy deyatelnosti studentov (na materiale izucheniya angliyskogo yazyka) [Problem-communicative approach as a factor in enhancing the cognitive activity of students (based on the study of English)]. Extended abstract of Candidate's thesis. Ufa: p. 19 [in Russian].

Itelson L.B. (1970). Problemy sovremennoy psikhologii ucheniya [Problems of modern psychology of learning]. Moscow: Znaniye, Issue 4, p. 56 [in Russian].

Izarenkov D.I. (1990). Bazisnyye sostavlyayushchiye kommunikativnoy kompetentsii ihformirovaniye na prodvinutom etape obucheniya student nefilologov [Basic components of communicative competence and their formation at the advanced stage of teaching non-philological students]. N.p.: Russian language abroad, № 4 pp. 56 -57, [in Russian].

Jacobovits L.A. (2008). Foreign Language Learning: A Psycholinguistic Analysis of the Issues Newbury House language series XXY, 0912066024, 9780912066028, $336 \mathrm{p}$.

Karapetova M.N. (2000). Formirovanie pedagogicheskoi kompetentnosti prepodavatelya obrazovatelnogo uchrezhdeniya [Formation of pedagogical competence of a teacher of an educational institution]. Extended abstract of candidate's thesis. Moscow: p. 192 [in Russian].

Kennedy, C. \& R. Bolitho (1984). English for Specific Purposes. London: Macmillan.

Kenzhebekov B.T. (2002). Suchshnost I struktura professionalnoi kompetentnosti spetsialista [The essence and structure of the professional competence of a specialist]. Higher School of Kazakhstan. N.p.: \#2, pp. 81-84 [in Russian]. 
Khmelevskaya T. \& Yarnatovskaya V. (1990). Russkiy yazyk I spetsialnost [Russian language and specialty]. Moscow: pp. 378-379 [in Russian].

Kitaigorodskaya G.A. (1986). Metodicheskiye osnovy intensivnogo obucheniya inostrannym yazykam [Methodical bases of intensive teaching of foreign languages]. Moscow: Publishing house of Moscow state University, p.176 [in Russian].

Klimenko U.N. (2003). Sotsialno-pedagogicheskaya kompetentnost spetsialistov sotsialnoi sfery [Social and pedagogical competence of specialists in the social sphere]. N.p.: Pedagogy. \# 2, pp. 22-28 [in Russian].

Komarkov V.S. (1975). Taktika doprosa [Interrogation tactics]. Kharkov: a textbook, Izd-vo Kharkovskogo yuridicheskogo instituta, pp. 19 -23 [in Russian].

Konstitutsiya Respubliki Kazakhstan (30.08.1995, 1998). (s izmeneniyami ot 07.10.1998 g.) [Constitution of the Republic of Kazakhstan dated August 30, 1995 (as amended on October 7, 1998) [in Russian].

Kozhin A. M., Krylova O. A. \& Odintsov V. V. (1982). Funktsionalnyye tipy russkoy rechi [Functional types of Russian speech] Uchebnoyeposobiyedlyastudentov [A textbook for students]. Moscow: Vysshayashkola, p.223, p.81 [in Russian].

Kozhina M.N. (1966). O spetsifike khudozhestvennoy I nauchnoy rechi v aspekte funktsionalnoy stilistiki [On the specifics of artistic and scientific speech in the aspect of functional stylistics]. Perm, p.35 - 36 [in Russian].

Krubelye M. (1989). Obrazovanie - 50/50: Opyt slovarya novogo myshleniya [Education - 50/50: Vocabulary Experience of New Thinking]. Moscow: Progress [in Russian].

Kudryashov Yu. A. \& Gurvich P.B. (1982). Metodicheskaya tipologiya tem ustnoy rechi // Problemy razvitiya umeniy inoyazychnoy ustnoy rechi [Methodical typology of topics of oral speech // Problems of development of foreign language oral speech skills]. Vladimir: Izd-vo VGPI, p.18 [in Russian].

Kunanbayeva S.S. (2005). Sovremennoye inoyazychnoye obrazovaniye: metodologiya i teorii [Modern foreign language education: methodology and theory]. Almaty: Edelweiss, p. 264 [in Russian].

Kuzmina N.V. (1990). Professionalizm lichnosti prepodavatelya [Professionalism of the teacher's personality]. Moscow [in Russian].

Kuzmina N.V. (1982). Metody sistemnogo pedagogichevskogo issledovaniya [Methods of systemic pedagogical research]. Leningrad: p.311 [in Russian].

Lapidus B.A. (1989). Nekotoryye aspekty sovremennogo sostoyaniya metodiki obucheniya inostrannym yazykam kak nauki [Some aspects of the current state of teaching methods of foreign languages as a science]. Collection of scientific works. Moscow: Publishing house of Moscow state University, Issue326, pp. 14 -20 [in Russian]. 
Larsson, J. (2001). Problem - based learning: A possible approach to language education. Polonian Institute, Jagiellonian University, March 2001. Retrived from www. Nada.kth.se/-jla/docs/PBL.pdf.

Law of the Republic of Kazakhstan (1999, 2001). "Ob obrazovanii» ["On Education"] No. 389-1 (as amended in accordance with the Laws of the Republic of Kazakhstan) 484-1; No. 207-II [in Russian].

Lenin V.I. (N.d.). Poln. sobr. Soch. [Collection], Moscow: vol. 29, pp. 152 - 153, [in Russian].

Leontyev A. A. (1974). Psikhologicheskiye problemy massovoy kommunikatsii. [Psychological problems of mass communication]. Moscow: Nauka, p. 111 [in Russian].

Leontyev A. N. (1972). Problemy razvitiya psikhiki [Problems of the development of the psyche]. Moscow: Publishing house of Moscow state University, 3rd ed. p. 575 [in Russian].

Leontyev A.A. (1999). Myslitelnyye protsessy v usvoyenii inostrannogo yazyka // Pedagogika i psikhologiya [Thought processes in mastering a foreign language]. Pedagogy and psychology. Moscow: p.75 [in Russian].

Leontyev A.N. (1959). Problemy razvitiya psikhiki [Problems of the development of the psyche]. Moscow, p.232 [in Russian].

Leontyev A. A. (1974). Psikhologicheskiye problem y massovoy kommunikatsii [Psychological problems of mass communication]. Moscow: Nauka [Science], p.33, [in Russian].

Loghman Ansarian, Fateme Shir Mohammadi (2018). Problem-Based Learning in Action: review of empirical studies. Pertanika Journal of Social Sciences \& Humanities, Volume 26, (T), Kuala Lumpur, Malaysia, Pages 13-32.

Lomov B.F. (1981). Problema obshcheniya $v$ psikhologii [The problem of communication in psychology]. Moscow: Nauka, n.p. [in Russian].

Lomov B.F. (1979). Kategoriya obshcheniya I deyatelnosti. Voprosy filosofii [Category of communication and activity. Problems of Philosophy]. Vol. 8, p. 13 [in Russian].

Lvov M. R. (1988). Slovar-spravochnik po metodike russkogo yazyka. [Dictionary - reference book on the methodology of the Russian language], Moscow: Prosveshcheniye [Education], p.215, 9, [in Russian].

Luke C. (1999). Cyborg pedagogy in cyborg culture. Teaching Education. pp. 6972. Retrieved from http://relayweb.hwwilsonweb.com.

Mamardashvili M. (2019). Vilnusskie lektsii po sotsialnoi filosofii [Vilnius lectures on social philosophy]. Saint - Petersburg. Retrieved from great words, http://emosurf.com/post/6535 [in Russian].

Markova A.K. (1996). Psihologiya professionalizma [Psychology of 
professionalism]. Moscow: International humanitarian fund "Znanie", p. 308 [in Russian].

Maslow H. (2013). A Theory of Human Motivation. [ Reprint of 1943 Edition]. /Theory-Human-Motivation-Abraham-Maslow/dp/1614274371

Matveeva T.V. (1990). Funktsionalnyye stili $v$ aspekte tekstovykh kategoriy: Sinkhronno-sopostavitelnyy ocherk [Functional styles in the aspect of text categories: Synchronous-comparative sketch]. Sverdlovsk: Publishing House of Ural University, p. 172 [in Russian].

Melnik A.E. (1989). Problemnyy podkhod pri obuchenii ustnomu vyskazyvaniyu [Problematic approach in teaching oral expression]. Psychology and methods of teaching foreign languages at the university: Sb. nauch. tr. Moscow: p.37 [in Russian].

Milashkina E.A. (1988). K probleme otboratekstovi form kontrolya na nachalnom etape obucheniya vtoromu inostrannomu yazyku. Voprosy ratsionalizatsii otbora uchebnogo materiala dlya prepodavaniya inostrannykh yazykov $v$ vuze [On the problem of the selection of texts and forms of control at the initial stage of teaching a second foreign language. Questions of rationalization of the selection of educational material for teaching foreign languages at the university]. Smolensk: Publishing house of SGPI named after K. Marx, p.95 [in Russian].

Minaeva S. A. (1991). Osnovy masterstva ustnogo vystupleniya. Kak podgotovit polemista [Fundamentals of oral presentation: How to prepare a polemicist]. Perm, p.5, 29 [in Russian].

Molchanovskii V.V. (2002). Obshchee I chastnoe v professionalnoi kompetentsii prepodavatelya, uchaushchego russkomu yaziku uchashchihsya - nefilologov. Traditsii I nivstsii $v$ professionalnoi deyatelnosti prepodavatelya russkogo yazika kak inostrannogo [General and specific in the professional competence of a teacher, teaching the Russian language to students-nonphilologists. Traditions and innovations in the professional activity of a teacher of Russian as a foreign language]. Moscow [in Russian].

Naumov A. (1996). Method of a specific situation in teaching management. n.p.: Management, Vol. 2, p.25 [in Russian].

Nazarbayev N.A. (2012). Strategiya “Kazakhstan - 2050”. Novyi politicheskiy kurs sostoyavshegosya gosudarstva: Poslanie Prezidenta Respubliki Kazahstan Lidera natsii N.A.Nazarbaeva narodu Kazakhstana [Strategy "Kazakhstan 2050". New political course of the established state. Message from the President of the Republic of Kazakhstan - Leader of the Nation N.A.Nazarbayev to the people of Kazakhstan]. Retrieved from https://www.akorda.kz/ru/ official_documents/strategies_and_programs in Russian].

Noguera J. (2004). Las competencias básicas. Retrieved from http://www.xtec.cat/ ilopez15/materials/competenciesbasiques/lascompetencia 
sbasicasnoguera.pdf (Consultado el 7-2-2015).

Obrecht D. (1976). Fundamentals of language and the basics of teaching a language: the need for their interaction. [Methods of teaching foreign languages abroad]. Moscow: Progress, Issue. 2. pp. 24 -35.

Obshchaya ritorika [General rhetoric] (1986). Dubois J., Peer F., Trinon A. \& others (trans. From French), Avelicheva A.K. (ed.), Moscow: Progress, p.392 [in Russian].

OCDE (2003). La definición y selección de competencias clave. Resumenejecutivo.

OECD PROJECT (2005). Definition and Selection of competencies (DESECO). Retrieved from http: //www.deseco.admin.ch/bfs_deseco/en/index/02.html.

O’Leary D.E. (2003). Technologies for knowledge assimilation. [In: Holsapple C.W. (eds) Handbook on Knowledge Management]. International Handbooks on Information Systems, vol 2. Springer, Berlin, Heidelberg. https://doi.org/10.1007/978-3-540-24748-7_2

Order of the Minister of Education and Science of RK (2018). "On Approval of the mechanism for targeted training of Master's and PhD Doctors in Basic Higher Educational Institutions (with amendments and additions dated October 8, 2018), No. 235 [in Russian].

Order of the Ministry of Internal Affairs of the Republic of Kazakhstan (1998) "On approval of the Regulations on Postgraduate Studies, on Doctorate Studies, on the preparation of doctoral and candidate dissertations outside of doctoral studies and postgraduate studies at higher educational institutions of the Ministry of Internal Affairs of the Republic of Kazakhstan", No. 416, p. 9, 1 [in Russian].

Orumbayev Zh. B. (2004). Tolkovaniye norm prava v pravoprimenitel'nom protsesse [Interpretation of the norms of law in the law enforcement process]. Extended abstract of candidate's thesis, - Almaty: p. 119 [in Russian].

Palmer A. (1970). Teaching Communication. Language Learning. Vol. 20-31, pp. 55-68.

Paris Communiqué (2018). Retrieved from http://ehea.info/Upload/document/ ministerial_declarations/EHEAParis2018_Communique_final_952771.pdf

Passov E.I. (1983). Teoreticheskiye osnovy obucheniya inoyazychnomu govoreniyu. [Theoretical foundations of teaching foreign language speaking]. Voronezh: Voronezh State University, n.p. [in Russian].

PISA (2003). Available at: http://www.pisa.oecd.org/science/struct.htm.

Ponomarenko B.T. (n.d.). Razvitie professionalnoi kompetentsii menedzherov [Development of professional competence of managers]. Retrieved from http:// www.rabe.ru/new/rus/m32_31.htm [in Russian].

Prikaz MVD RK (2004) “O Kontseptsii razvitiya vedomstvennogo obrazovaniya 
Ministerstva vnutrennih del Respubliki Kazahstan" [On the Concept for the development of departmental education of the Ministry of Internal Affairs of the Republic of Kazakhstan]. \# 397

Prikaz MVD RK (2016). «Ob utverzhdenii Programmy razvitiya vedomstvennogo obrazovaniya Ministerstva vnutrennikh del Respubliki Kazakhstan na 20172020 gody» [Order of the Ministry of Internal Affairs of the Republic of Kazakhstan "On approval of the Program for the development of departmental education of the Ministry of Internal Affairs of the Republic of Kazakhstan for 2017-2020"], No. 600 pp. 30, 33 [in Russian].

Problems of complicity in the criminal legislation of the Republic of Kazakhstan. (2016). Syllabus. Karaganda: KYUI MIA RK named after B. S. Beisenova [in Russian].

Public Security Management. Syllabus. (2016). Karaganda: KYUI MIA RK named after B. S. Beisenova, p.36 [in Russian].

Randall Z. (2002). "Idealnoye» obucheniye pismu I pedagogicheskaya ierarkhiya. // Peremena: Mezhdunarodnyi zhurnal o razvitii myshleniya cherez chteniye $i$ pismo ["Ideal" teaching writing and pedagogical hierarchy // Change: International journal on the development of thinking through reading and writing]. Vol. 3 (4), pp. 8-15 [in Russian].

Ramos M. M. \& Luque G. (2010). A competence-based constructivist tool for evaluation. Cultura y Educación, 22 (3), pp. 329-344.

Rassel B. (2014). Filosofskii slovar razuma, materii I morali [Philosophical Dictionary of Mind, Matter and Morals]. Retrieved from Open Road Media, ISBN 1497675707, 9781497675704.

Ray M. (2018). 8 Deadliest Wars of the 21st Century. https://www.britannica. com/list/8-deadliest-wars-of-the-21st-century

Rhalmi, M. (2010). Communicative activities. pages.com/blog/english-website

Ricoeur P. (2001). Hermeneutics and Social Science Method. Lecture. Moscow: p.3.

Riziab, C.E., Najafipour, M., Haghani, F., Dehghan, S. (2013). The effect of the using the brainstorming method on the academic achievement of students in grade five in Tehran. [2nd World Conference on Educational Technology Researches - WCETR2012], Elsevier, Procedia - Social and Behavioral Sciences 83 (2013) 230 - 233.

Robinson P.C. (1991). ESP Today: A Practitioner's Guide. N.Y.: Prentice Hall.

Romenville M. (2004). Competence approach in university education: objectives, benefits, boundaries [Presentation, AMUE seminar "LMD Diplomas in Competence Terms and Presentation of the Diploma Supplement"], Paris.

Rubinstein S.L. (1989). Osnovy obshchey psikhodogii [Fundamentals of General Psychology]. Moscow: Pedagogy, Vol. 1, p. 485 [in Russian]. 
Rubinshtein S. L. (1946). Osnovy obshchey psikhologii [Fundamentals of General Psychology]. Moscow: Soc. EKGIZ, p.213 [in Russian].

Safonova L. Yu. (2015). Primeneniye interaktivnykh form obucheniya. [Application of interactive forms of teaching].Velikie Luki: Methodical instructions, pp. 16, 39 [in Russian].

Safonova V.V. (1996). Izucheniye yazykov mezhdunarodnogo obshcheniya $v$ kontekste dialoga kultur I tsivilizatsiy [Studying the languages of international communication in the context of the dialogue of cultures and civilizations]. Voronezh: Istoki [in Russian].

Saharova N.S. (1999). Kategorii "kompetentnost" $I$ "kompetentsiya" $v$ sovremennoi obrazovatelnoi paradigm [Categorie "competence" in the modern educational paradigm]. Vestnik OGU, \# 3 [in Russian].

Salkhanova Zh. Kh. (2004). Yazykovaya kompetentnost yuristov[Linguistic competence of lawyers]. Yevraziya, Vol. 6 [in Kazakh].

Saville - Troike M. (2003). The Ethnography of Communication. Oxford: Blackwell Publishing Ltd.

Schneider, R. M., Krajcik, J., Marx, R. W., \& Soloway, E. (2002). Performance of students in project-based science classrooms on a national measure of science achievement. Journal of Research in Science Teaching, 39, 410-422.

Scarcella R.C. \& Oxford R. L. (1992). The Tapestry of Language Learning, Boston, Heinle \& Heinle Publishers.

Schultz R. A. (2007). The challenge of assessing cultural understanding in the context of foreign language instruction. Foreign Language Annals, Vol. 40(1).

Semenova S.N. (2007). Formirovanie professionalnoi kompetentsii inostrannyh studentov, obuchaushchihsya $\mathrm{v}$ vyshih hudozhestvennyh uchebnyh zavedeniyah Rossii [Formation of professional competence of foreign students studying in higher art educational institutions in Russia]. Extended abstract of candidate's thesis, Moscow: p. 30, 173[in Russian].

Semenova M.V. (2005). Pedagogicheskie usloviya formirovaniya professionalnoi kompetentnosti buduchshih pedagogov v vuze [Pedagogical conditions for the formation of professional competence of future teachers at the university]. Extended abstract of candidates thesis. Karaganda: p. 152 [in Russian].

Serova T.S. (1989). [Theoretical foundations of teaching professionally oriented reading in a foreign language in a non-linguistic university]. Extended abstract of Doctor's Thesis. Leningrad, p. 57 [in Russian].

Shaposhnikov K.V. (2006). Kontextnyi podhod v processe formirovaniya professionalnoi kompetentnosti buduchshih lingvistov - perevodchikov [Contextual approach in the process of forming the professional competence of future linguists-translators]. Extended abstract of candidate's thesis. IoshkarIola: p.26 [in Russian]. 
Shchepkina E. (2002). Uchebnyy protsess: vzglyad iznutri [The educational process: a view from the inside]. Higher education in Russia. Vol.5 [in Russian].

Shergina N.N. (1983). Rol zritelnogo obraza $v$ organizatsii predmetnogo soderzhaniya rechevogo soobshcheniya [The role of the visual image in the organization of the subject content of the speech message. M: Nauchnye trudy. Izd-vo Mosk. gos. ped. in-ta in. yaz. im. M.Toreza, Issue 207, p.131[in Russian].

Shin \& Azman, (2012). Problem-based Learning in English for a Second Language Classroom: Students' Perspectives, International Journal of Learning, Vol 18 (6), DO10.18848/1447-9494/CGP/v18i06/47648JO. Retrieved from https://www.researchgate.net/publication/286947340

Shmelev D. N. (1977). Sovremennyy russkiy yazyk. Leksika [Modern Russian. Lexicon]. Uchebnoye posobiye dlya studentov [Textbook for students], Moscow: Prosveshcheniye, p.89 [in Russian].

SJUR Bergan (Council of Europe) Qualifications. (2007). Introduction to a Concept, Council of Europe Publishing, Part 1. Approaching a complex reality.

Skvortsov L.I. (1980). Teoreticheskiye osnovy kultury rechi. [Theoretical foundations of speech culture], Moscow: Science, p.109, [in Russian].

Slastenin V.A. (1996 a,b). O sovremennyh podhodah $k$ podgotovke uchitelya. [On modern approaches to teacher training]. The technology of psychological and pedagogical preparation of a teacher for educational activities. Barnaul: p. 167 [in Russian].

Slesareva I. L. (1980). Problemy opisaniya i prepodavaniya russkoy leksiki [Problems of description and teaching of Russian vocabulary]. Moscow: p. 159, 5 [in Russian].

Stanford Encyclopedia of Philosophy (2018). Hans - Georg Gadamer. [First published Mon Mar 3, 2003; substantive revision Mon Sep 17, 2018]

State compulsory standard of postgraduate education "Master" as amended by the Resolution of the Government of the Republic of Kazakhstan dated May 13, 2016, No. 292

Stoof A., Marten R., Van Merrienboer J. \& Bastiens T. (2002). The Boundary Approach of Competence: a Constructive Aid for Understanding and Using the Concept of Competence. Human Resource Development Review, 1 (3), pp. 345365

Suharno, M. (2017). Language in scientific writing. [Fakultas Sastra]. Retrieved from: https://core.ac.uk/download/pdf/11704727.pdf

Taubayeva Sh.T. (2005). Formirovanie etnopedagogicheskoi kompetentnosti uchitelei $v$ sisteme povysheniya kvalifikatsii [Formation of ethnopedagogical competence of teachers in the system of advanced training]. Proceedings from scientific and practical conference "Ethnopedagogy in the education system: experience, problems and prospects". Atyrau: pp. 204-206 [in Russian]. 
Tepley J. (2014) Properties of Being in Heidegger's Being and Time. International Journal of Phylosophycal Studies. Vol.22, No.3, 461-481, http://dx.doi.org/10.1080/09672559.2014.913382

The Oxford dictionary of the Christian church (3 reved.) (2009). Edited by F.L.Cross and E.A. Livingstone. Oxford University Press. eISBN: 9780199566716

The Interpretation Act. (1980). Case study: Fothergill v. Monark Airlines Ltd. HL.

The Interpretation Act. (1857). Case study: Lord Wensleysale in Grey v. Pearson HL.

The Interpretation Act. (1977). Case study: Miller v. Jackson, QB 966, p.22.

Tienson J. (1983). Linguistic Competence. Transactions of the Nebraska Academy of Sciences and Affiliated Societies. 259. https://digitalcommons.unl.edu /tnas/259

Tipovaya programma po inostrannym yazykam dlya vuzov MVD RK [Typical program in foreign languages for universities of the Ministry of Internal Affairs of the Republic of Kazakhstan], (2002), Astana: GUKUZ MIA RK.

Truth and Method (1989), 2nd rev. ed. (1st English edn, 1975, trans. by W, GlenDoepel, ed. by John Cumming and Garret Barden), revised translation by J. Weinsheimer and D.G. Marshall, New York: Crossroad. [Since the appearance of the 2nd revised edition in 1989, Truth and Method has been republished in various formats by Continuum, and more recently Bloomsbury, without substantive change to the text, but unfortunately without maintaining any uniformity of pagination.]

Tsvetkova Z.M. (1971). Problema aktivizatsii intellektualnoy deyatelnosti $v$ protsesse obucheniya $v$ yazykovom vuze. Nauchno-metodicheskaya konferentsiya "Lingvo-psikhologicheskiye problem obosnovaniya metodiki prepodavaniya inostrannykh yazykov $v$ vysshey shkole» [The problem of activating intellectual activity in the process of teaching at a language university]. Scientific and methodological conference "Linguistic and psychological problems of substantiating the methodology of teaching foreign languages in higher education". Abstracts. Moscow: p. 339 [in Russian].

Turisbekov Z.K. (2004). Legal newspaper. Astana: Febr. [in Russian].

Uteubayeva E.A. (2010). Formirovaniye inoyazychnoy kommunikativnoy kompetentsii yuristov v sisteme poslevuzovskogo obrazovaniya [ Formation of foreign language communicative competence of lawyers in the system of postgraduate education] Extended abstract of Candidate's thesis. Karaganda: p. 65 [in Russian].

Uteubayeva E.A., Kultanova Zh.M. \& Pastushenko T.A. (2012). Creative thinking as a basis of foreign language learning by the methods of project activities. Prague: Education and science without borders. Vol. 3. №5 (1/2012), pp.115- 
117.

Uteubayeva E.A. (2005). Cautious, but drastic steps in the optimization of the language competence of magisters-lawyers at a postgraduate stage. [The Science: Theory and Practice: Materials of international scientific-practical conference, Volume 17. Pedagogical Sciences], Praha: Publishing House «Education and Science», pp. 46-51.

Uteubayeva E.A. (2012). Multimediynoye uchebnoye posobiye po angliyskomu yazyku dlya magistrantov yuridicheskikh spetsialnostey [Multimedia English Language Study Guide for Master Students in Law]. Astana: E - book. Ministry of Justice of Republic of Kazakhstan, № 952.

Uteubayeva E.A. (2012). Kontrolno-obuchayushchaya programma dlya izucheniya yazykovykh distsiplin $v$ sisteme vuzovskogo i poslevuzovskogo obrazovaniya [Control and educational program for the study of language disciplines in the system of higher and postgraduate education]. Astana: E - book. Ministry of Justice of Republic of Kazakhstan, № 610.

Uteubayeva E.A., Zhetpisbayeva B.A. \& Pastushenko T.A. (2011). The theoretical model of foreign language communicative competence of lawyers in system of postgraduate education. Scientific journal "Education and Science without borders", Prague: \# 2 (4), p. 86.

Uteubayeva E.A. (Dildyaeva E.A.) (2002). Drugs. Uchebno-metodicheskoe posobie po angliiskomu yazyku [Workshop], Karaganda: Part 1,2.

Vihansky O.S. (2001). Metod konkretnykh situatsiy. [Method of specific situations]. Moscow: p.21 [in Russian].

Vitt N.V. (1973). Metodika prepodavaniya inostrannykh yazykov v vuze. [Methods of teaching foreign languages at the university]. Moscow: Publishing house of Moscow state university, Vol. 3, Part 1, p. 24 [in Russian].

Voprosy psihologii. Questions of Psychology. (1990) Scientific collection. Moscow, p. 239.

Voronina G.V. (1994). Metodika obucheniya chteniyu autentichnykh tekstov molodezhnykh sredstv massovoy informatsii uchashchikhsya zavershayushchey stupeni obshchego srednego obrazovaniya s uglublennym izucheniyem inostrannogo yazyka (na materiale nemetskogo yazyka) [Methods of teaching the reading of authentic texts of youth mass media to pupils of the final stage of general secondary education with an in-depth study of a foreign language (based on the material of the German language): Extended abstract of Candidate's thesis. Moscow: Moscow State University [in Russian].

Vvedenskii V.N. (2003). Modelirovanie professionalnoi kompetentnosti pedagoga. [Modeling the professional competence of a teacher]. N.p.: Pedagogy, Vol. 10, pp. 51-58 [in Russian].

Westera W. (2001). Competences in Education: a Confusion of Tongues. Journal of 
Curriculum Studies, 33 (1), pp. 75-88.

William Ellet (2020). The Case Study Handbook: A Student's Guide. Harvard Business Publishing Education.

World Declaration on Higher Education for the 21st Century: Approaches and Practical Measures (1998). Innovative approaches in education: critical thinking and co-operation. Paris: UNESCO, Art. 9.

Yakobson P. M. (1969). Psikhologicheskiye problemy motivatsii povedeniya cheloveka [Psychological problems of human behavior motivation]. Moscow: Nauka [in Russian].

Yakobovits L.A. (1976). Metodika prepodavaniya inostrannykh yazykov za rubezhom [Methods of teaching foreign languages abroad]. Moscow: Progress, pp, 144 -145 [in Russian].

Yew E.H.\& Goh K. (2016). Problem-Based Learning: An Overview of Its Process and Impact on Learning. Health Prof. Educ.; 2:75-79. Retrieved from http: doi: 10.1016/j.hpe.2016.01.004.

Yore, D. L., Bisanz, L. G., \& Hand, M. B. (2013). Examining the literacy component of science literacy: 25 years of language arts and science research. International Journal of Science Education, 25, 689-725

Zakon Respubliki Kazakhstan «O yazykakh v Respublike Kazakhstan» (1997). [Law of the Republic of Kazakhstan "On languages in the Republic of Kazakhstan"]. № 151-1[in Russian].

Zhang J., Lin H. (2018). An Empirical Study on the Influence of PBL Teaching Model on the Critical Thinking Ability of Non-English Majors. JLTR. Retrieved from http: 9:1293-1300. doi: 10.17507/j1tr.0906.19.

Zheng R., Perez J., WilliamsonJ. \& Flygare J. (2008). WebQuests as perceived by teachers: implications for online teaching and learning. Journal of Computer Assisted Learning, 24, 295-304.Retrieved from http: doi: 10.1111/j.1365-2729

Zhetpisbayeva B.A., Uteubayeva E.A. (2012). Angliyskiy yazyk dlya magistrantov yuridicheskikh spetsialnostey [English for postgraduates in Legal specialties]. Uchebnoye posobiye [Workshop], Karaganda: Izdatelstvo Kargu, p. 13.

Zohar, A., \& Dori, Y. J. (2003). Higher order thinking skills and low-achieving students: Are they mutually exclusive? The Journal of the Learning Sciences, $12,145-181$.

Zweigert, K., Kotz, H. (1998). Introduction to comparative Law. Oxford University Press, Oxford, UK, 10, Tony Weir trans., 3rd ed. 
ELMIRA UTEUBAYEVA

\title{
CONCEPTUAL FRAMEWORK FOR TEACHING PROFESSIONAL FOREIGN LANGUAGE (CASE OF LSP FOR LAW) \\ /for universities studying English/
}

\author{
Monograph \\ Copying of content is not permitted except for personal and internal use, to the extent permitted \\ by national copyright law, or under the terms of a CC BY-NC 4.0 License. \\ English \\ First Edition \\ Editor: \\ DZINTRA ILIŠKO, Professor, Dr \\ Daugavpils University, Latvia \\ Institute of Humanities and Societal Sciences, Centre of Sustainable Education \\ Pages: 180 \\ Format: $60 \times 84 / 8$ \\ 22,5 печатни коли
}

Publisher:

Publishing House “ACCESS PRESS”, Bulgaria, 2021

32 Alexander Burmov Str., 5000 Veliko Tarnovo, Bulgaria

Email: office@access-bg.org

http://www.access-bg.org

ISBN 978-619-91511-3-6

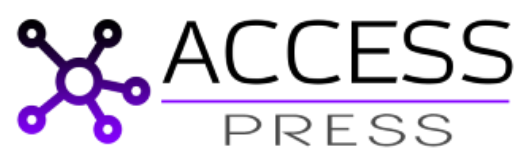

